\title{
ABSTRACTS \\ Faculty Presentations at The International Congress on Controversies in Stem Cell Transplantation and Cellular Therapies (COSTEM)
}

\author{
Leukemia Supplements (2012) 1, S8-S45; doi:10.1038/leusup.2012.3
}

\section{Prevention of leukemia relapse with donor lymphocyte infusions (DLI) for minimal residual disease in acute leukemia Andrea Bacigalupo \\ Divisione di Ematologia e Trapianto di Midollo, Ospedale San Martino, Genova, Italy \\ Address: Andrea Bacigalupo, MD, Divisione Ematologia 2, Ospedale San Martino, Largo Rosanna Benzi 10,16132 Genova, Italy, Phone: +39 010 355469; Fax: +39 010355583. \\ E-mail: andrea.bacigalupo@hsanmartino.it}

Relapse remains a significant issue in acute leukemia patient undergoing an allogeneic hemopoietic stem cell transplant (HSCT). Once a patient with acute leukemia has relapsed post-transplant, cell therapy, such as donor lymphocyte infusions (DLI) is usually ineffective and survival is poor. One may thus conclude that acute leukemia is less sensitive to so called graft versus leukemia (GvL), especially if compared with chronic myeloid leukemia (CML), where DLI alone can induce durable long term molecular remissions (1). However, several reports suggest a strong protection exerted by chronic graft versus host disease (GvHD) in acute lymphoid (ALL) and myeloid (AML) leukemia $(2,3)$ : the original Weiden paper (2) included mainly ALL patients, and a more comprehensive analysis of the Seattle data, confirmed a strong GvL effect of GvHD in patients with acute leukemia (3). Several additional studies have confirmed that patients with acute leukemia developing GvHD have a significantly lower risk of relapse as compared to patients with no GvHD $(4,5,6)$. This finding is further supported by two prospective randomized studies testing two different doses of Cyclosporin early after transplant, in adults and children with acute leukemia: both showed reduced leukemia relapse with low dose CsA $(7,8)$ This confirms that GvHD protects against leukemia relapse and that early post-transplant events are crucial. How do we reconcile the strong GvL effect in acute leukemia, with the lack of effect of DLI once hematologic relapse has occurred. Is it a question of tumour burden?

If this is the case, then DLI may be effective in preventing leukemia relapse, if given at the time when leukemia burden is low, the so called minimal residual disease (MRD). In keeping with this hypothesis are studies using DLI In patients with mixed chimerism (9) and a few patients with MRD+ ALL converting to MRD- ALL after DLI (10). We have been running a program of monitoring of minimal residual disease (MRD) following an allogeneic hemopoietic stem cell transplant (HSCT) in acute leukemia (AML).

Relapses occur early after HSCT in patients with acute leukemia: in a data set of 175 relapses in acute leukemia following an allogeneic HSCT, 9\% occurred within 60 days, 25\% between day 61 and 120, $15 \%$ between day 121 and $180,30 \%$ between day 181 and 365 , and $21 \%$ beyond 1 year (unpublished). Therefore half of the relapses occur within 6 months and the majority within one year. Monitoring of MRD in the first 12 months post-HCT should be picking up a significant number of relapses.

We therefore opened a study of molecular monitoring of MRD post transplant, to test whether MRD positivity predicts relapse and, if so, if there is enough time between the two events for immune intervention, including DLI. We now report the first 80 patients on this program. MRD was monitored in 80 patients with acute lymphoid (ALL, $\mathrm{N}=44$ ) or myeloid ( $\mathrm{AML}, \mathrm{n}=36$ ) leukemia, undergoing an allogeneic hemopoietic stem cell transplant. MRD markers were IgH-VDJ and TCR gene re-arrangement for ALL, and Wilms Tumour (WT1) expression for AML. The overall cumulative incidence $(\mathrm{Cl})$ of MRD positivity was $45 \%$ and the $\mathrm{Cl}$ of hematologic relapse was $24 \%$ (36\% in MRD + vs $16 \%$ in MRD- patients, $p=0.03$ ). The median interval from transplant to first MRD positivity was day +120 , and to hematologic relapse, day +203 . Patients could be divided in 3 MRD groups: MRD$(n=44), M R D+$ given donor lymphocyte infusions (DLI) $(n=17)$ and MRD+ not given DLI $(n=19)$ : leukemia relapse in these 3 groups was respectively $16 \%, 6 \%$ and $63 \%(p<0.0001)$; the actuarial 5 year survival was $78 \%, 80 \%$ and $26 \%$ ( $p=0.001)$. In multivariate COX analysis, MRD group predicted relapse $(p<0.0001)$ and survival $(p=0.01)$, together with disease phase and chronic graft versus host disease. In MRD+ patients, DLI protected against relapse $(p=0.003)$ and improved survival $(p=0.01)$.

Causes of death were as follows: in MRD- patients $(n=44)$ there were 3 death due to relapse and 3 due to GvHD; in MRD+DLI+ patients 2 patients died of GVHD and one of relapse; in MRD+DLI- patients 9 died of leukemia and 1 died of GvHD and one of heart failure.

In conclusion, minimal residual disease detected post-transplant is a significant predictor of relapse in patients with acute leukemia. Treatment of MRD with donor lymphocyte infusions, appears to protect against leukemia relapse, although caution with DLI dosing needs to be used, because of the potential risk of graft versus host disease.

\section{References}

1. Weisser M, Tischer J, Schnittger S, Schoch C, Ledderose G, Kolb HJ. A comparison of donor lymphocyte infusions or imatinib mesylate for patients with chronic myelogenous leukemia who have relapsed after allogeneic stem cell transplantation. Haematologica 2006; 91:663-6.

2. Weiden PL, Sullivan KM, Flournoy N, Storb R, Thomas ED. Antileukemic effect of chronic graft-versus-host disease: contribution to improved survival after allogeneic marrow transplantation. N Engl J Med 1981; 304(25):1529-33.

3. Sullivan KM, Weiden PL, Storb R, Witherspoon RP, Fefer A, Fisher L, Buckner CD, Anasetti C, Appelbaum FR, Badger C et al. Influence of acute and chronic graft-versus-host disease on relapse and survival after bone marrow transplantation from HLA-identical siblings as treatment of acute and chronic leukemia. Blood 1989; 73:1720-8.

4. Passweg JR, Tiberghien P, Cahn JY, Vowels MR, Camitta BM, Gale RP, Herzig RH, Hoelzer D, Horowitz MM, Ifrah N, Klein JP, Marks DI, Ramsay NK, Rowlings PA, Weisdorf DJ, Zhang MJ, Barrett AJ. Graft-versus-leukemia effects in $T$ lineage and $B$ lineage acute lymphoblastic leukemia. Bone marrow Transplant 1998 Jan; 21(2):153-8.

5. Zikos $P$, van Lint MT, Lamparelli $T$, Gualandi $F$, Occhini $D$, Bregante $S$, Berisso G, Mordini N, Incagliato M, Fugazza G, Sessarego M, Bacigalupo A: Allogeneic hemopoietic stem cell transplantation for patients with high risk acute lymphoblastic leukemia: favorable impact of chronic graftversus-host disease on survival and relapse. Haematologica 1998; 83: 896-903.

6. Nordlander A, Mattsson J, Ringden O, Leblanc K, Gustafsson B, Ljungman P, Svenberg P, Svennilson J, Remberger M. Graft-versus-host disease is associated with a lower relapse incidence after hematopoietic stem cell transplantation in patients with acute lymphoblastic leukemia. Biol Blood Marrow Transplant 2004 Mar; 10(3):195-203.

7. Bacigalupo A, van Lint MT, Occhini $D$, Gualandi $F$, Lamperelli T, Sogno $\mathrm{G}$, Tedone $\mathrm{E}$, Frassoni F, Tong J, marmont AM. Increased risk of leukemia 
relapse with high-dose cyclosporine $A$ after allogeneic marrow transplantation for acute leukemia. Blood 77; 1423-1428, 1991.

8. Locatelli F, Zecca M, Rondelli R, Bonetti F, Dini G, Prete A, Messina C, Uderzo C, Ripaldi M, Porta F, Giorgiani G, Giraldi E, Pession A. Graft versus host disease prophylaxis with low-dose cyclosporine-A reduces the risk of relapse in children with acute leukemia given HLA-identical sibling bone marrow transplantation: results of a randomized trial. Blood 2000 Mar 1; 95(5):1572-9.

9. Bader $P$, Kreyenberg $H$, Hoelle W, Dueckers $G$, Handgretinger $R$, Lang $P_{1}$ Kremens B, Dilloo D, Sykora KW, Schrappe M, Niemeyer C, Von Stackelberg A, Gruhn B, Henze G, Greil J, Niethammer D, Dietz K, Beck JF, Klingebiel T. Increasing mixed chimerism is an important prognostic factor for unfavourable outcome in children with acute lymphoblastic leukemia after allogeneic stem-cell transplantation: possible role for preemptive immunotherapy? J Clin Oncol 2004 May 1; 22(9):1696-705.

10. Miglino M, Berisso G, Grasso R, Canepa L, Clavio M, Pierri I, Pietrasanta D, Gatto S, Varaldo R, Ballerini F, Verdiani S, Casarino L, De Stefano F, Sessarego M, Dominietto A, Raiola AM, Bregante S, Di Grazia C, Gobbi M, Bacigalupo A: Allogeneic bone marrow transplantation (BMT) for adults with acute lymphoblastic leukemia (ALL): predictive role of minimal residual disease monitoring on relapse. Bone Marrow Transplant 2002; 30:579-585.

\section{Should all MRD positive patients receive allo SCT? Peter Bader}

\section{University Children's Hospital Frankfurt am Main, Germany}

During the past decades progress has been made in the treatment of childhood acute lymphoblastic leukemia (ALL) by conventional chemotherapy. Even at relapse about one third of patients can be salvaged by chemo/radiotherapy and stem cell transplantation. However, relapse is the principal cause of treatment failure after allogeneic stem cell transplantation (SCT) for high risk childhood ALL. Specific methods aimed at preventing relapse comprise the use of intensive treatment prior to transplant, optimal transplant preparative regimens and post-transplant interventions with targeted or immunologic therapies.

Numerous retrospective reports have shown that minimal residua disease (MRD) immediately prior to conditioning represented the strongest predictor for post-transplant relapse. In 1998, Knechtli et al. from Bristol showed that patients entering transplantation with a high MRD load of $>10-3$ did not survive their disease, whereas a portion of patients with low level disease $(<10-3)$ survived. MRD negative patients had the best chance of survival. Consequently, this group could show the importance of MRD as a prognostic factor for the success of allogeneic SCT. A retrospective analysis of German data largely confirmed these results in a series of 45 children: Patients with high level MRD at time of transplant (>10-3) were cured rarely. However, even in these high risk patients, additional immunotherapy post-transplant could eradicate residual disease. These findings were confirmed by further retrospective studies, all demonstrating that patients, who entered transplantation with high MRD load, faced an extreme risk to develop subsequent relapse after transplantation. Nevertheless these studies showed some limitations since these investigations were performed retrospectively and included heterogeneous patient cohorts, transplanted in CR1 and $>C R 2$ after having received different front line treatment and different conditioning regimens. Though, confirmation of these retrospective results by prospective trials was needed before MRD quantification could be introduced as criterion for strategical decisions in clinical protocols.

The ALL-REZ BFM Study Group initiated such a prospective trial in order to investigate the prognostic impact of pre-transplant MRD load in a defined group of pediatric patients, who received allogeneic SCT in second or third remission. A multivariate Cox proportional hazard model for pEFS was fitted including the covariates sex, age at relapse, remission status, time point of relapse, immune phenotype, site of relapse, stem cell donor, T-cell depletion, time to SCT, GVHD and MRD prior to SCT. In this model, only MRD emerged as independent prognostic factor (Wald Chi-square $=0.006$ ). . . with an MRD load of $\geq 10-4$ had a risk ratio of $2.45(95 \% \mathrm{Cl} 1.30-4.62)$ of suffering an adverse subsequent event above patients with a level <10-4. [50] Thus, results of three major retrospective analyses could be confirmed, which suggested the prognostic impact of MRD prior to SCT in rather heterogeneous patient cohorts with less standardized methods.

Although MRD positive patients have a poor outcome after transplantation, it is these patients only curative treatment. Therefore all these patients should receive transplant, however, MRD assessment allows adaptation of post transplant treatment guidance in these ultra high risk patients.

\section{Should CR be target of therapy in myeloma? Bart Barlogie}

\section{University of Arkansas for Medical Sciences, USA}

Historically, it is important to remember that, until the introduction of high-dose melphalan therapy, fewer than $5 \%$ of myeloma patients achieved complete remission (CR). However, as CR increments translated into event-free survival (EFS) and overall survival (OS) benefits, many strategies have since been employed toward realizing this early treatment objective, including tandem transplants, consolidation and maintenance interventions, and, more recently, the upfront addition of novel agents, such as thalidomide and bortezomib. When employed as maintenance, novel agents imparted ongoing $C R$ increments translating into extensions of EFS and $O S$.

The Arkansas team has reported that failure to achieve $C R$ did not impact outcomes adversely when patients had a documented preceding smoldering course, had a GEP signature of monoclonal gammopathy of undetermined significance (MGUS), or presented with the CD2 molecular subtype of myeloma. We recently examined the impact on survival outcomes of both attaining CR status and the duration of $C R$, applying time-dependent variables for $C R$ onset and its sustenance as well as landmark techniques. Sustaining CR for at least 3 years was associated with superior outcome while patients attaining but losing CR status within 3 years had a dismal prognosis (linked in part to high-risk genomics). Those failing to enter CR had an intermediate prognosis. None of the above considerations account for imaging-defined CR. In fact, we and others have reported on the prognostic consequences of MRI-defined focal lesions that resolve with a significant time delay of 18 months beyond immunofixation (IF)-derived CR status, attesting to the increasingly recognized heterogeneity of myeloma, which includes a non-secretory component that contributes to late relapses often involving extramedullary sites.

The issue of curability in multiple myeloma has been debated with enthusiasm by some and challenged by others pursuing the objective of chronic disease control over disease eradication. While this issue is compounded by significant co-morbidities in the elderly myeloma patient, we have reported recently that Kaplan-Meier plots of $\mathrm{CR}$ duration, EFS, and OS are compatible with cure models in our Total Therapy (TT) programs. These cure models assume that a fraction of patients have long-term remission duration, EFS, or OS, while the remainder fail at a constant rate. Figure $1 \mathrm{~A}$ depicts EFS curves for 3 TT protocols, demonstrating significant cure-fraction estimates for TT1, TT2 (both control and experimental [added thalidomide] arms), and TT3 (added bortezomib); among this last group, significant curefraction estimates were limited to the GEP-defined low-risk patients (Figure 1B). The data are jointly depicted in Figure 1C, along with significance comparisons among the protocols. Figure 1D depicts, for all 3 protocols, Kaplan-Meier plots for OS and EFS as well as CR duration. We recognize that, even with purportedly greater cure potential provided by a graft-v-myeloma effect of allogeneic stem cell transplants, relapses do occasionally occur as late as 15 and 20 years. The possibility exists that such recurrences ensue from long-dormant focal lesion sites or represent progression from other clones of a 
genetically heterogeneous MGUS. Such exceptions, however, cannot be used as an argument against the curability of myeloma in general as they pertain also to childhood ALL and other malignancies with accepted cure potential.

The following should be considered in ongoing clinical investigations of myeloma therapy:

- A comprehensive genomic/genetic classification of myeloma is needed to determine whether CR implications are myeloma risk- or subtype-specific.

- Given the focal nature of bone marrow involvement of most myeloma cases, MRI and PET should become an integral part of major trials assessing quality of response on outcomes, as imaging-defined $C R$ may outweigh myeloma protein-defined CR.

- The distinct possibility exists that the clinical consequences of $C R$ vary not only according to myeloma genetics and risk, but are also influenced by specific treatments applied.

- While tumor cytoreduction is important for extending survival of patients with all cancers, the absence of achieving $C R$ in myeloma should not frighten patients, as attainment of a preceding MGUS state can be postulated as the underlying mechanism.

Figure 1: Modeling cure fractions (CF) from EFS plots.

A: Total Therapy 1 (TT1, left panel), Total Therapy 2 (TT2, center panel: no thalidomide $v$ thalidomide arms), Total Therapy 3 (TT3, right panel)
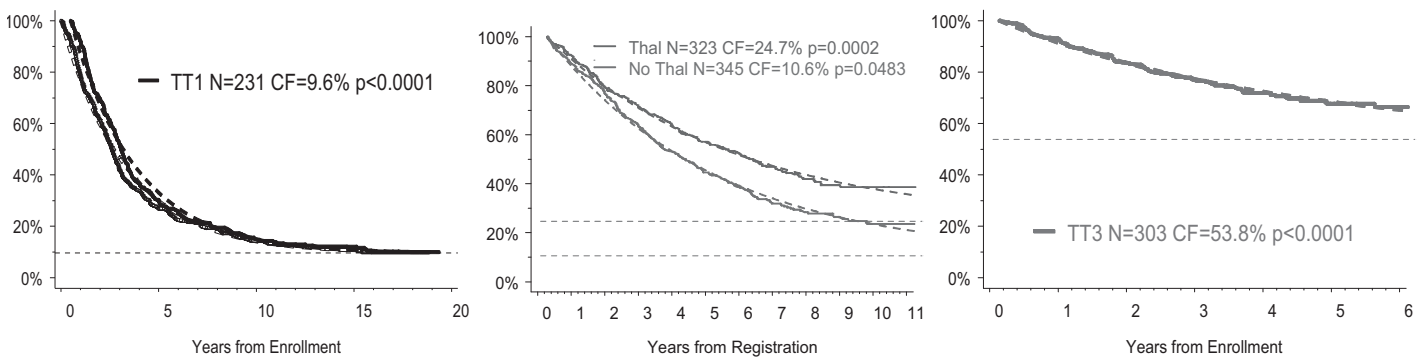

\section{B: Total Therapy 3 according to gene array-defined risk.}

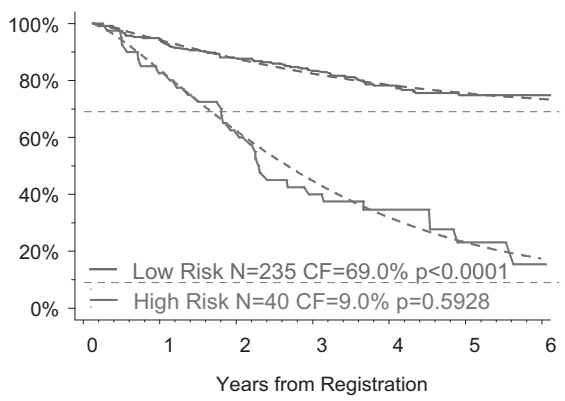

C: Summary of cure fraction data in Total Therapies 1, 2, and 3 with comparison of successive trials (table).

Cure Fraction by Protocol

EFS

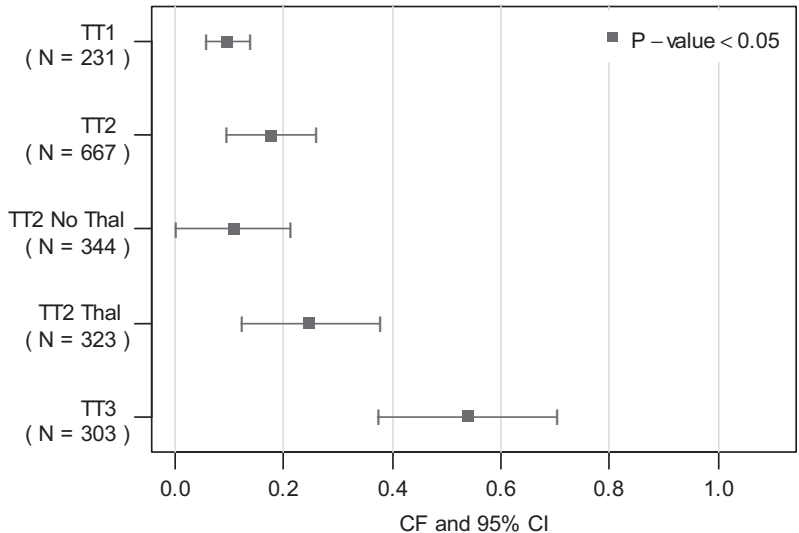

\begin{tabular}{|lc|}
\hline EFS Comparison & P Value \\
\hline TT1 vs. TT2 & 0.0863 \\
\hline TT1 vs. TT2 No Thal & 0.8543 \\
\hline TT1 vs. TT2 Thal & 0.0257 \\
\hline TT1 vs. TT3 & $<0.0001$ \\
\hline TT2 Thal vs. TT2 No Thal & 0.0927 \\
\hline TT2 vs. TT3 & 0.0001 \\
\hline TT2 No Thal vs. TT3 & $<0.0001$ \\
\hline TT2 Thal vs. TT3 & 0.0060 \\
\hline
\end{tabular}

tapproximate P values from t-tests 
D: Kaplan-Meier Plots for Total Therapy Protocols TT1, TT2 without and with thalidomide, and TT3.

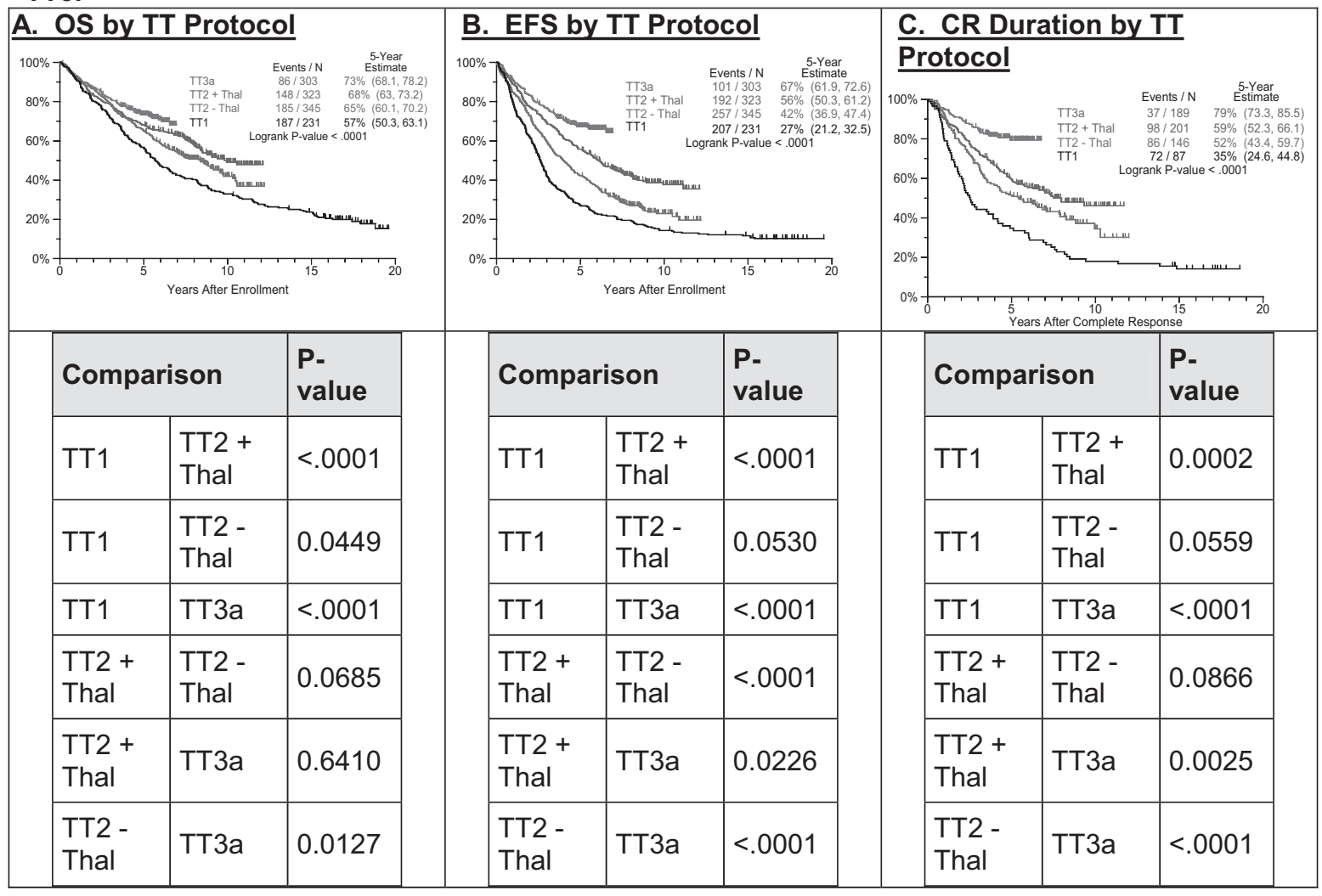

\section{Autologous stem cell transplantation as first line treatment in multiple myeloma (For the NO part) \\ Joan Bladé and Laura Rosiñol}

Institut of Hematology and Oncology, Department of Hematology, IDIBAPS, Hospital Clinic, Barcelona, Spain

Autologous stem cell transplantation (ASCT) is considered the gold-standard for younger patients with multiple myeloma (MM). However, whether the apparent benefit comes from the "all" population or it only results from certain subsets of patients needs to be revisited.

The first studies on ASCT in MM were performed in patients with advanced refractory disease. Although the response rate was high, the median EFS and OS were extremely short. So, it is evident that patients with relapsed/refractory myeloma are not the "ideal" candidates for ASCT. However, ASCT should be considered whenever possible in patients with sensitive relapse (Fermand et al., Blood 1998). There is a general belief that patients with primary refractory MM are the most likely to benefit from early ASCT (Alexanian et al., Blood 1994). However, for a meaningful interpretation of the data the criteria for "refractory" disease should be clearly defined as well as whether or not the primary therapy has been the "optimal" one. Importantly, the two categories of patients with primary refractory disease (i.e., primary unresponsive with progressive disease or with minimal response or no change but without clinical progression - nonresponsive/nonprogressive -) should be analyzed separately. In fact, in the Spanish experience, the median survival of 31 patients with primary unresponsive progressive disease who underwent an ASCT was only 21 months (review, Bladé et al., Blood 2010).
In the up-front setting the results of trials comparing ASCT versus conventional chemotherapy have not been uniform across the studies. In fact, three of the five fully published trials showed no benefit of ASCT in terms of EFS and OS. There are a number of reasons that might have accounted for the discrepancies among these trials (review, Bladé et al., Blood 2010). The achievement of post-transplant $C R$ is the crucial step for a long-lasting response and prolonged survival after ASCT in MM and the achievement of $\mathrm{CR}$ is highly correlated with the sensitivity to the induction therapy measured by the M-protein at the time of transplant. Conversely, in patients not reaching $C R$, the benefit of ASCT is questionable. Thus, patients with suboptimal response to the induction therapy are unlikely to achieve CR after ASCT and, consequently, to benefit from high-dose therapy. Other question is whether or not patients already in CR after induction therapy benefit from ASCT. In this sense, the MD Anderson has consistently reported that patients who achieve CR with primary therapy and who do not receive an ASCT have the same prolonged PFS and OS as those obtaining CR after ASCT (Alexanian et al, BMT 2001; Wang et al, BMT 2010). In the same direction, a Mayo Clinic study showed that patients who were in CR at the time of ASCT had a similar outcome as those achieving CR after ASCT only (Dingli et al, JCO 2007). Thus, whether patients in CR with primary therapy would benefit from ASCT intensification is unknown.

In summary, ASCT is of no benefit in patients with refractory myeloma aa well in those with suboptimal response to primary therapy. The role of ASCT in patients in CR after primary therapy is unknown. The patients most likely to benefit from ASCT are those with highly sensitive disease to the initial induction therapy (question mark for those achieving $\mathrm{CR}$ with primary treatment). 


\section{Post-autologous stem cell transplantation consolidation in multiple myeloma \\ Joan Bladé and Laura Rosiñol}

Institut of Hematology and Oncology, Hematology Department, IDIBAPS, Hospital Clinic, Barcelona, Spain

High-dose therapy followed by hematopoietic stem cell support (ASCT) is the gold-standard in the treatment of younger patients with multiple myeloma (MM). Ideally, the whole treatment program should include an effective induction regimen and highdose therapy/stem cell support likely followed by consolidation and maintenance. The greatest survival benefit is achieved in patients attaining immunofixation negative complete remission. With the use of conventional chemotherapy induction regimens, the post-transplant CR rate is about 35\%, the medial survival of 6 years and the proportion of patients in continued CR beyond 10 years ("operational cure") from ASCT is about $10 \%$. In an attempt to improve these results, novel agents have been incorporated in the current transplant programs. Triple induction regimens such as bortezomib/adriamycin/dexamethasone (PAD) and bortezomib/ thalidomide/dexamethasone (VTD) with pre- and post-transplant CR rates ranging from 19 to $31 \%$ and from 43 to $46 \%$, respectively, are superior to the so-called double regimens consisting of thalidomide/dexamethasone (TD) or bortezomib/dexamethasone (VD). The Total Therapy III, incorporating bortezomib in the VTD-PACE induction, followed by tandem ASCT, one year of VTD consolidation and 2 years of TD maintenance, resulted in 56\% CR rate at 2 years from the initiation of therapy (Barlogie et al., BJH 2007). It is evident that the achievement of IFE negative CR is the first step for a long-lasting response and prolonged survival after ASCT in MM Bladé et al, Blood 2010). However, the achievement of a serological response should no longer be the ultimate goal in the treatment of MM. In fact, the Arkansas group has recently reported, applying a time-dependent variable methodology, on the importance of not only achieving $\mathrm{CR}$ but also of sustaining $\mathrm{CR}$, in patients included in Total Therapy programs I, II and III (Hoering et al, Blood 2009). On the other hand, sequential minimal residual disease (MRD) measurements by multiparameter flow cytometry (Paiva et al, Blood 2008) or molecular studies (Ladetto et al, JCO 2010) can be crucial to guide from what level of MRD a certain treatment is still needed. In order to achieve the lowest MRD status, post-ASCT consolidation may become an essential component of therapy. In this regard, the Italian group has reported that $18 \%$ of patients in at least VGPR after ASCT achieved molecular remission by qualitative and quantitative PCR reaction with 4 cycles of VTD consolidation (Ladetto et al, $J C O, 2010)$. Encouraging improved responses with VTD consolidation, including molecular responses, after ASCT have been reported in abstract form in other studies (Cavo et al, ASH 2010; Terragna et al, ASH 2010; Roussel et al, ASH 2010). An improved response rate with single agent bortezomib (Mellqvist et al, ASH 2009) as well as with single agent lenalidomide (Attal et al, ASH 2010) consolidation after ASCT has also been reported.

\section{Is specific T-cell therapy targeting mHag useful? No Martin Bornhäuser}

\section{University Hospital Dresden, Germany}

Minor histocompatibility antigens (mHag) were described more than two decades ago as peptides spanning amino acid polymorphisms in cellular proteins differentially expressed in various tissues, processed and presented in a HLA-restricted fashion. The main interest initially had been to define potential antigens which could discriminate between graft-versus-leukemia effects and harmful graft-versus-host reactions after allogeneic stem cell transplantation (SCT). Subsequently, efforts have been undertaken to generate and expand cytotoxic T cell clones (CTL) or lines with specificity for $\mathrm{mHag}$ expressed on tumor targets in order to facilitate adoptive cell-based therapies after allogeneic SCT. Although the potency of $\mathrm{mHag}$-specific T cells has been described after allogeneic SCT, it is currently unclear what the specific therapeutic approach based on these cells would be like and off-target effects including lung-toxicity have been described for T cell clones expanded ex-vivo using IL-2 and CD3/CD28 beads. But still, the number of known immunotherapeutically relevant $\mathrm{mHags}$ is small. Both investigators in the Netherlands and in the US have been able to use patient-specific $\mathrm{mHag}$ targeted CTLs in patients with leukemia relapsing after allogeneic SCT. Because of the limitations in $\mathrm{MHC}$ restriction and $\mathrm{mHag}$ expression this approach may be feasible in only $20-30 \%$ of patients undergoing allogeneic SCT. New hope is therefore coming from genetically engineered $T$ cells which express either a transgenic $T$ cell receptor of given specificity of even more advanced chimeric antigen receptors (CARs). Although anti-CD19-CARs have been developed and are clinically used with success, other diseases like $A M L, M D S$ and $C M L$ in which only a limited set of target surface antigens can be used, may be less prone to this strategy. In these diseases, T cell lines transduced with TCR combined with protein-or peptide based vaccination strategies may be warranted. With the mentioned caveats in mind, it remains currently unclear whether genetically engineered or ex-vivo expanded CTLs will be able to compete with the rapidly evolving field of monoclonal antibodies including bi-specific diabody constructs which do not require extensive ex-vivo manipulation of cells. Extensive cellular manipulations pose major regulatory hurdles to the clinical application of mHag-specific T cell preparations as an evolving strategy. Future research will have to focus on in-vitro tests predicting the efficacy of a given cell-based approach in various diseases, especially when ex-vivo expansion of cells is involved. It remains to be awaited whether'personalized'T cell therapies can compete with companydriven 'blockbuster' approaches.

\section{Is double cord blood to be preferred to a single unit?} Claudio G Brunstein

Associate Professor, Division of Hematology, Oncology, and Transplantation, Department of Medicine and Bone Marrow Transplant Program, University of Minnesota, Minneapolis, MN

The double umbilical cord blood (dUCB) transplantation platform was introduced approximately 10 years ago and one of its goals was to allow adults and larger adolescents to undergo UCB transplantation by additive effect of the nucleated cell dose (NCD) of two units. The promising data initially reported by the University of Minnesota has now been reproduced by independent investigators in the US and Europe. The indication and selection of dUCB grafts varies according to institutional practice. Candidates for dUCB transplantation are typically patients who do not have a suitable single UCB as defined by a minimum cryopreserved nucleated cell dose of $2.5 \times 10^{7} / \mathrm{kg}$ of recipient actual body weight. While the minimum required cell dose for each of the two units composing the graft ranges from 1 to $2 \times 10^{7} / \mathrm{kg}$ of recipient actual body weight, all centers will allow a maximum of 2 HLA-mismatches to the recipient. Whether the 2 donor units need to be HLA-matched to each other is a matter of debate; most centers will only allow 2-3 HLAmismatches between the 2 units, considering HLA A and B at antigen level and HLA-DRB1 at allele level. After dUCB transplantation neutrophil engraftment is $85-95 \%$ at a median time of 26 days, but may be shorter after reduced intensity conditioning in part due to autologous reconstitution; the incidence of platelet engraftment is $60-70 \%$. While chimerism from both units may be observed earlier on, with mixed chimerism in reduced intensity setting, a single unit predominates long term in the majority of patients. Acute GVHD has been reported to be as high as $60 \%$ after dUCB transplantation, but center that use ATG report rates as low as $30 \%$, similar to single UCB. TRM ranges from $20-40 \%$ and is higher in patients who 
take longer to achieve neutrophil recovery and those with co-morbidities. Studies suggest that the risk of relapse is relatively lower after myeloablative dUCB, and DFS is around $35-50 \%$ depending on disease stage. In the reduced intensity setting the risk relapse ranges from $35-40 \%$ and LFS $30-45 \%$, with the higher risk of treatment failure among patient with advanced stage leukemia. Studies comparing dUCB with single UCB or adult donors in the myeloablative setting suggest similar long-term outcomes. In the comparison with single UCB, engraftment, TRM and chronic GVHD were similar, but acute GVHD was higher after dUCB (Rocha et al., ASH 2010). Relapse was lower and LFS better for recipients of dUCB in CR1, but was similar for single and double UCB for patients in CR2-3. Recent data suggest that, as compared to dUCB, single UCB transplantation by intra-bone marrow injection (IBMI) may provide similar neutrophil engraftment, better platelet engraftment, lower acute GVHD, similar TRM and relapse, and better DFS (Rocha et al., ASH 2010). In a comparison with related and unrelated adult donors in the myeloablative setting, dUCB recipients had lower neutrophil engraftment and acute GVHD, similar chronic GVHD, and higher TRM and lower relapse (Brunstein et al., Blood 2010). Overall, LFS at 5 years was similar for all donor types. In contrast, in the reduced intensity setting the outcomes of DUCB and PBSC from unrelated donors were similar with LFS of 30-35\% (Brunstein et al., ASH 2010). In summary, available data demonstrate that dUCB transplantation leads to outcomes similar to recipients of single UCB and related and unrelated adults. Data on single UCB by IBMI seems promising but requires confirmation. Thus, for patients who need an allograft but lack of suitable adult donor or need urgent of transplantation and that do not have an adequate single UCB should be considered for a dUCB graft.

\section{Should cord blood unit be selected according to HLA or cell-based? \\ Claudio G Brunstein}

Associate Professor, Division of Hematology, Oncology, and Transplantation, Department of Medicine and Bone Marrow Transplant Program, University of Minnesota, Minneapolis, MN

The less stringent HLA-matching is one of the positive aspects of umbilical cord blood (UCB) transplantation that allows us to find grafts for patients who lack a suitable related donor or required in which case would not be able to wait the time it takes to obtain unrelated adult donor (URD) graft. Most transplant centers will accept 0-2 mismatches considering matching HLA-A and $B$ at the antigen level and HLA-DRB1 at the allele level when selecting UCB, while requiring 7-8/8 allele level matching at $\mathrm{HLA}-\mathrm{A}, \mathrm{B}, \mathrm{C}$ and $\mathrm{DRB} 1$ for URD. Despite a less stringent HLA-matching UCB transplantation has reproducibly shown provides long-term outcomes similar to URD sources. A notable exception has been hematopoietic recovery that is typically delayed and is less complete after UCB transplantation. Numerous investigators have established that the main factor influencing hematopoietic engraftment of UCB is cell dose, and current practice recommendations are that a minimum cell dose of $2.5 \times 10^{7} / \mathrm{kg}$ of actual recipient body weight is required to proceed to UCB transplantation; the cell dose requirement is higher for patients with non-malignant diseases. However, data on the impact of HLA-matching on the outcome of UCB transplantation dates from the late 1990's when it was shown that poorly matched grafts led to worse outcomes. It should be pointed early on that the use of UCB units with 3 or more mismatches should be discouraged. Data on the impact of HLA-matching on UCB transplantation outcome is best studied in pediatric patients because the large number of patients, allowing the study of interactions between cell dose and the different levels of HLA-mismatch. Thus, the question is once the $2.5 \times 10^{7} / \mathrm{kg}$ cell dose threshold is achieved, should we go for the largest possible UCB unit with 0-2 HLA-mismatches or should we choose the best HLA-matched unit above that cell dose? Of course, this question can only be asked in cases where multiple potential units are available. Registry-based and prospective studies have shown that recipients of HLA-matched UCB units have better engraftment, lower TRM and superior DFS. In children receiving UCB units with $1 \mathrm{HLA}$-mismatch data shows that those who received the higher cell doses had better engraftment and lower TRM. In contrast, a cell dose effect is not clear in recipients of UCB units with $2 \mathrm{HLA}$-mismatches that have lower engraftment, higher TRM but lower risk of relapse. Despite most UCB grafts having 1-2 HLA-mismatches, no effect on acute or chronic GVHD has so far been demonstrated. Notably, in recipients of UCB grafts with 1-2 HLA-mismatches survival is similar as higher risk of TRM with 2 HLA-mismatches is offset by lower risk of relapse. The effect of HLA-matching in adult UCB transplantation is less clear due to the smaller number of patients for and adequately powered study and the fact that most adults receive units with $2 \mathrm{HLA}$-mismatches. Data available does not support considering matching UCB at the HLA-C locus. In contrast, data in single UCB suggests KIR-ligand mismatching leads to lower relapse and better DFS. However, considering HLA-C or KIR-ligand matching would typically limit UCB graft options, and would only be practical in patients with several potential UCB graft units. Recent data on the effect of the non-inherited maternal allele is encouraging and could potentially increase the number of suitable units for individual patients, but that information is not yet readily available. Last, double UCB adds another layer of complexity to the understanding of effects of HLAmatching and outcomes. In summary, 6/6 HLA-matched unit with $\mathrm{NCD} \geq 2.5 \times 10^{7} / \mathrm{kg}$ is the preferred but rarely available. When only units with 1-2 HLA-mismatches are available, not only cell dose but also the patient's disease needs to be considered as those with nonmalignant disease have a higher risk of graft rejection.

\section{Gene therapy for genetic diseases: an important alternative option for genetic diseases such as primary immunodeficiencies and Fanconi Anemia \\ Marina Cavazzana-Calvo 1,23,4, Salima Hacein-Bey-Abina ${ }^{1,2,3,4}$ and} Alain Fischer ${ }^{3,4,5}$

'Department of Biotherapy, Hopital Necker Enfants-Malades; ${ }^{2}$ Centre d'Investigation Clinique en Biothérapie, Groupe Hospitalier Universitaire Ouest, Inserm/Assistance Publique - Hopitaux de Paris; ${ }^{3}$ Inserm Unit 768, Fondation Imagine; ${ }^{4}$ Faculté de Médecine, Université Paris Descartes, Sorbonne Paris Cité and ${ }^{5}$ Pediatric Immunology \& Hematology Unit, Hôpital Necker Enfants-Malades, AP-HP, Paris, France

The concept of gene therapy emerged as a way of correcting monogenic inherited diseases by introducing a normal copy of the mutated gene into at least some of the patient's cells. Although this concept has turned out to be quite complicated to implement, it is in the field of primary immunodeficiencies (PIDs) that proof of feasibility has been undoubtedly achieved. There is now a strong rationale in support o gene therapy for at least some PIDs, as discussed in this presentation.

Many PIDs are lethal diseases. In the absence of treatment, severe combined immunodeficiencies (SCIDs) cause death within the first year of life. Many other combined deficiencies of adaptive immunity (such as Wiskott Aldrich syndrome [WAS] and diseases causing hemophagocytic lymphohistiocytosis $[\mathrm{HLH}]$ can also be fatal in young infants. Allogeneic hematopoietic stem cell transplantation (HSCT) can cure many of these disorders by replacing the diseased hematopoietic lineages with normal ones. These results prove that transplantation of normal hematopoietic stem cells or their progenitors can correct a large variety of PIDS of the adaptive and innate immune systems. However, HSCT is associated with several serious adverse events, including the toxicity of myeloablative chemotherapy and, above all, the consequences of the potential immune conflict between donor and recipient. The latter can result in either graft failure or, conversely, graft-versus-host disease, a cause of 
serious morbidity and mortality. In the context of human leukocyte antigen (HLA) mismatch, the risk for immune conflict can be alleviated by removing donor T cells from the stem cell inoculum. However, this type of approach is still marred by the risk for graft failure and prolonged immunodeficiency before donor-derived immunity develops. Although advances in SHCT methodology may provide better solutions to these problems, it is clearly legitimate to search for alternative, gene-based approaches.

Most PIDs display Mendelian inheritance, so that addition of a normal copy of the mutated gene can correct the deficiency provided that the right cells are targeted and transgene expression is appropriate. At present, the pathophysiological mechanisms of many PIDs have been worked out and provide further clues to the use of gene therapy. Furthermore, the technical aspects of ex vivo gene transfer into hematopoietic progenitors have been improved over the years by using appropriate cytokine cocktails to put cells in cycle when retrovirus (RV) are used or in G1 of the cell cycle when lentivirus (LV) are used, and thus facilitate vector integration. These developments made gene therapy an important alternative option for some PIDs at least.

\section{Should haplo-SCT be performed with T-cell depletion? No Ciceri Fabio}

Hematology and Bone Marrow Transplantation Unit, IRCCS H San Raffaele, Milano, Italy

Background and Aim: Rapamycin, in contrast to calcineurin inhibitors, allows $\mathrm{T}$ regulatory cell (T-regs) proliferation while inhibits effector T cell expansion. We investigated the safety of infusion of T-cell repleted unmanipulated PBSC from haploidentical donor with a combination of Rapamycin, Mycophenolate and ATG as GvHD prophylaxis, to preserve early T-regs function (TrRaMM study, Eudract 2007-5477-54).

Patients and Methods: Since 2007, seventy-nine patients (pts) underwent stem cell transplantation (SCT) for high risk leukemias and lymphomas. Median age was 47 years (range 14-69). At transplantation 10 pts were in early phase, and 69 in dvanced phase. Median comorbidity index score was 2 . The conditioning regimen included Treosulfan (14 g/m $\mathrm{m}^{2}$ for 3 days), Fludarabine $\left(30 \mathrm{mg} / \mathrm{m}^{2}\right.$ for 5 days) and an in-vivo T and B-cell depletion by ATG-Fresenius ( $10 \mathrm{mg} / \mathrm{kg}$ for 3 times) and Rituximab (a single $500 \mathrm{mg}$ dose). All pts received allogeneic unmanipulated PBSC from an HLA-haploidentical related donor. GvHD prophylaxis consisted of Rapamycin (target level $8-15 \mathrm{ng} / \mathrm{ml}$, till day +60$)$ and MMF $(15 \mathrm{mg} / \mathrm{kg}$ tid till day +30 ).

Results: All patients engrafted and all but eight were in disease remission at first marrow evaluation on day +30 . 1-year cumulative incidence of grade 2-4, grade 3-4 aGvHD and cGvHD were 22, 9 and $21 \%$ respectively. 1 -year cumulative incidence of TRM and relapse incidence were $25 \%$ and $44 \%$ respectively. After a median follow-up of 391 days, 1-year OS was $41 \%$. Immunoreconstitution was fast and sustained with a median 221 circulating CD3+cells/ $\mathrm{A} \mu \mathrm{L}$ (range 43-1690) from day 30. The immunoreconstitution was polarized towards central memory (CD45RA-CD62L+ cells 32.7\%), IL-2 producing cells (IL-2+ cells $26.2 \%$ ). We detected high levels of CD4+CD25+CD127-FOXP3+ T-regulatory cells (up to $30 \%$ of circulating CD4+ T lymphocytes) starting from day 30 . These cells were able to suppress in vitro proliferation of autologous effector cells demonstrating to be regulatory $T$ cells.

Conclusions: Rapamycin-Mycophenolate-ATG are effective as GvHD prevention in T-cell replete unmanipulated haploidentical peripheral SCT and are associated with an early T-cell immunoreconstitution characterized by the in-vivo expansion of earlydifferentiated T cells and T-regs. Further studies are warranted to gain insight on the role of Rapamycin as platform for exploitation of T-regs in allogeneic HSCT from mismatched donors.
Transplantation for T-cell lymphoma (pro-allo position) Paolo Corradini and Anna Dodero

Division of Hematology and BMT, IRCCS Istituto Nazionale dei Tumori, University of Milano

Patients affected by mature T-cell lymphomas, other than anaplastic large cell lymphomas (ALCL) with anaplastic lymphoma kinase (ALK) expression, have a 5-year overall survival of approximately $25 \%$ with conventional chemotherapies. In fact, most of them do not benefit from anthracycline-based therapy as a part of their induction treatment or from abbreviated chemotherapy intervals (1). To improve the poor results obtained with conventional chemotherapy, autologous stem cell transplantation (auto-SCT) has been offered to these patients as consolidation of first remission or in relapsed disease. Different studies have shown that only patients in first complete remission appear to achieve long-term remission with auto-SCT (2). However, autoSCT gives none or minimal survival benefit in patients with refractory or relapsed disease (3). In the last ten years, allogeneic stem cell transplantation (allo-SCT) has been more frequently investigated in patients with relapsed disease and a graft-versus-T-lymphoma effect has been postulated. In 2004, we firstly reported encouraging results in a small pilot study: 12 of 17 PTCL patients were alive and in CR after alloSCT performed for relapsed disease (4). In addition, Le Gouill et al. recently analysed the long-term outcome of 77 patients with several subtypes of PTCL and observed an overall survival of 57\% at 5 years (5).

We now expanded our original observation and thus we have retrospectively evaluated the long-term outcome of 52 patients receiving allo-SCT for relapsed disease (6). Histologies were PTCLnot-otherwise specified $(\mathrm{n}=23)$, anaplastic large-cell lymphoma $(n=11)$, angioimmunoblastic T-cell lymphomas $(n=9)$ and rare subtypes $(n=9)$. Patients were allografted from matched related siblings $(n=33,64 \%)$ or alternative donors $(n=19,36 \%)$ following a reduced-intensity conditioning (RIC) regimens including thiotepa, fludarabine and cyclophosphamide. Most of the patients had chemosensitive disease $(n=39,75 \%)$ and $27(52 \%)$ failed a previous auto-SCT. At a median follow-up of 67 months, 27 of 52 patients are alive $(52 \%)$ and $25(48 \%)$ died $[n=19$ disease progression, $n=6$ nonrelapse mortality (NRM)]. The cumulative incidence $(\mathrm{Cl})$ of NRM was $12 \%$ at 5 -years. Extensive chronic graft-versus-host disease (GVHD) increased the risk of NRM ( $33 \%$ versus $8 \%, p=0.04)$. The $\mathrm{Cl}$ of relapse was $49 \%$ at 5 -years, influenced by disease-status $(p=0.0009)$ and treatment lines $(\mathrm{p}=0.007)$. Five years OS and PFS were $50 \%(95 \% \mathrm{Cl}$ $36 \%$ to $63 \%)$ and $40 \%(95 \% \mathrm{Cl}, 27 \%$ to $53 \%)$, respectively. Eight of 12 patients $(66 \%)$ who received donor-lymphocytes infusions for early disease progression had a clinical response. At multivariable analysis, refractory disease and age over 45 years were independent adverse prognostic factors. The long-term follow-up indicates that $\mathrm{RIC}$ allo-SCT is an effective and feasible salvage treatment with a better outcome for younger patients with chemosensitive disease. Future studies should be focused on incorporating new drugs and allo-SCT in the salvage setting of PTCL.

\section{References}

1. Vose J, Armitage J, Weisenburger D. International peripheral T-cell and natural killer/T-cell lymphoma study: pathology findings and clinical outcomes. J Clin Oncol 26: 4124-4130, 2008

2. Reimer $P$, Rüdiger $T$, Geissinger $R$ et al. Autologous stem-cell transplantation as first-line therapy in peripheral T-cell lymphomas: results of a prospective multicenter study. J Clin Oncol 27: 106-113, 2009

3. Rodríguez J, Conde E, Gutiérrez A et al. Grupo Español de Linfomas/Trasplante Autólogo de Médula Osea, Spanish Lymphoma/Autologous Bone Marrow Transplant Study Group. The adjusted International Prognostic Index and beta-2-microglobulin predict the outcome after autologous 
stem cell transplantation in relapsing/refractory peripheral T-cell lymphoma. Haematologica 92: 1067-1074, 2007.

4. Corradini P, Dodero A, Zallio F et al. Graft-versus-lymphoma effect in relapsed peripheral T-cell non-Hodgkin's lymphomas after reducedintensity conditioning followed by allogeneic transplantation of hematopoietic cells. J Clin Oncol 22: 2172-2176, 2004.

5. Le Gouill S, Milpied N, Buzyn A et al. Graft-versus-Lymphoma effect for aggressive T-cell lymphomas in adults: a study by the Société Francaise de Greffe de Moëlle et de Thérapie Cellulaire. J Clin Oncol 26: 2264-2271, 2008

6. Dodero A, Spina F, Narni F, Patriarca F, Cavattoni I, Benedetti F, Ciceri F, Baronciani $D$, Scimè $R$, Pogliani $E$, Rambaldi $A$, Bonifazi $F$, Dalto $S$, Bruno B, Corradini P. Allogeneic transplantation following a reduced intensity conditioning regimen in relapsed/refractory peripheral T-cell lymphomas: long-term remissions and response to donor lymphocyte infusions support the role of a graft-versus-lymphoma effect. Leukemia 2011 [E-pub ahead of print Sep 9]. doi: 10.1038/leu.2011.240.

\section{Can we prevent relapse by novel conditioning regimen? Yes Marcos de Lima}

\section{Anderson Cancer Center, USA}

The combination of chemical and physical agents used prior to autologous or allogeneic progenitor cell transplants is known as the preparative or conditioning regimen. In the allogeneic transplant setting, the preparative regimen should also facilitate engraftment, in addition to provide at least some direct anti-malignancy activity. The optimal conditioning regimen for most hematologic malignancies as well as for each transplant type has not been well defined. Choice of regimen often varies from one institution to another and there are strong, unresolved controversies in the field in terms of what constitutes the 'best' conditioning.

Comparison of approaches is made difficult by the fact that the doses and types of chemotherapy and radiotherapy are not homogeneous. To complicate matters, differences in supportive care, graftversus-host disease prevention, donor-recipient Human Leukocyte Antigen compatibility and stem cell source also should be considered when analyzing outcomes. Analyses of published results are therefore difficult to perform, and frequently the choice of a regimen is made on the basis of institutional and personal experience.

A significant fraction of treatment failures after allogeneic transplantation is related to relapse and toxicity of the preparative regimen. Traditional dose escalation may decrease relapse rates at the expense of increased toxicity and non-relapse mortality. This conundrum is made worse when treating patients that are beyond the $6^{\text {th }}$ decade of life, which is also the age where most of the malignant diseases treatable with allogeneic transplants have their peak incidence.

Therefore, improving preparative regimens is synonymous of decreasing toxicity while preserving or increasing anti-malignancy activity, without compromising the immunologic graft-versusleukemia/lymphoma effect.

I was charged with arguing in favor of progress made (and to be made) in both fronts (toxicity and efficacy), and in my presentation I will show evidence that would suggest the answer to this question is indeed a 'YES'.

\section{Allogeneic stem cell transplantation in elderly patients with MDS? No}

H Joachim Deeg

Fred Hutchinson Cancer Research Center, USA

Hematopoietic cell transplantation (HCT) is the only therapeutic modality that has been shown to have curative potential for patients with MDS, some of whom are surviving now more than 25 years after HCT. Progressive modifications of transplant conditioning regimens have resulted in significantly reduced toxicity and mortality associated with the transplant procedure, and even patients in the eighth decade of life have been transplanted successfully. However, graft-versus-host disease (GVHD) has remained a major obstacle to uniformly successful HCT, and the frequency of GVHD after reduced-intensity conditioning (RIC) transplant regimens is not significantly different from that with higher dose regimens as typically used in younger individuals. Front-line therapy for GVHD, both in its acute and chronic forms, still uses glucocorticoids, which are poorly tolerated in older patients. In addition to the increased risk of infections, morbidity related to musculo-skeletal effects interferes with the patients' level of activity and quality of life (QOL). Patients may spend the remainder of their life in the hospital or at skilled-care facilities and require extended rehabilitation. This may not at all be the goal of patients in the seventh or eighth decade of life, who would rather spend good quality time with their families. Even more importantly, preliminary data suggest that particularly patients with low or intermediate-1 risk MDS as determined by the International Prognostic Scoring System (IPSS), have a longer life expectancy if not undergoing HCT early in their disease course. This perspective is likely different in patients with advanced MDS whose life expectancy without HCT may be short, and who may want to consider HCT, despite the expected morbidity and mortality. However, even for this latter group of patients, it will be important to consider not only transplant survival statistics, but life expectancy related to medical conditions other than MDS, which are quite frequent in older individuals, and the inherent risk of mortality. If non-transplant therapy without significant morbidity and mortality can modify the natural disease course, the net gain for these patients may be higher without HCT. There appears to be agreement that it is not so much the chronologic but, rather, the biologic age of the patient that determines the probability of success with HCT. Therefore, careful evaluation of older patients may lead to the selection of all those for HCT who are biologically younger than, say 60 or 65 years. Finally, there are non-medical and even non-biologic factors that should be considered. The psychological and financial stress on families associated with HCT can be overwhelming. While discussions of the economic impact of HCT on the family are typically avoided, expenses associated with HCT may exhaust a family's reserves. We should ask whether this outcome - for the family - is justifiable if the probability of failure is high and it is likely that the patient will not regain productivity and QOL.

\section{Is it possible to separate GVHD and GVL effects? No} $\mathrm{H}$ Joachim Deeg

\section{Fred Hutchinson Cancer Research Center, USA}

Graft-versus-leukemia (GVL) effects and the resulting reduction of disease recurrence after allogeneic hematopoietic cell transplantation (HCT) have been described in clinical trials since the late $1970 \mathrm{~s}$. Typically, these studies showed that patients who developed GVHD in its acute or chronic form, had a lower incidence of disease recurrence than patients without clinical evidence of GVHD. Presumably anyone receiving allogeneic $\mathrm{HCT}$ will experience an allogeneic reaction of donor cells against host cells, without which eradication of the leukemic clone(s) would not be possible. Therefore, some will argue, we have already achieved our goal of separating GVHD from GVL effects since, obviously (and fortunately) a proportion of patients will not experience relapse even in the absence of clinical GVHD. However, the fact is that in 2011, we cannot achieve this goal consistently. Studies on the infusion of donor lymphocytes (DLI) in patients with disease recurrence after HCT have shown that reinduction of remission generally, albeit not always, was associated with manifestations of GVHD, often including myelosuppression. A major factor in the development of GVHD is, presumably, the transplant conditioning regimen. Cytotoxic radio-chemotherapy given in preparation for HCT profoundly alters gene expression 
profiles, which, combined with direct tissue injury, results in dysregulation of cytokines, chemokines and adhesion molecules, among others. This altered milieu will modify host and donor cell functions at several levels, and cannot be selectively controlled at present. T-cell depletion has generally been associated with a reduction in GVHD but an increase in relapse incidence. A recent analysis on the impact of alemtuzumab or thymoglobulin in patients conditioned with reduced-intensity regimens (generating a cytokine milieu different from that observed with high dose regimens) is consistent with those earlier findings. Interleukin (IL)-2 for example, has been used to sustain the function of cytotoxic T-cells as well as to induce regulatory T-cells (Treg) - how to separate one from the other? Type 1 interferon signaling appears to enhance CD8+ mediated GVHD in addition to GVT effects. The use of selective CD4+ or CD8+ T-cell depletion has yielded some interesting results, but has had only a minor impact. In more recent trials depletion of naïve (CD45RA+) T-cells has been postulated to prevent GVHD while allowing for GVL effects to take place; however, even with this approach GVHD does occur. Whether data on CD8+CD44 ${ }^{\text {hi }}$ T-cell mediated anti-lymphoma activity without GVHD can be extrapolated from murine models to the clinic, remains to be tested.

The use of donor T lymphocytes directed at minor histocompatibility antigens, in addition to practical and logistic problems, is hampered by the fact that many of these antigens are not restricted to hematopoietic tissues, and it is unlikely, therefore, that GVHD will be prevented. Infusion of Treg is an attractive approach; however, there are major concerns that these cells may suppress not only GVHD but also the GVL reaction.

Considerable work has been conducted on NK cells and their role in GVHD and GVL effects. Particularly in patients receiving HLAhaploidentical transplants mismatching for KIR ligands appears to be beneficial in achieving a GVT effect without inducing GVHD. However, the success of this approach may depend upon the patient's diagnosis, and controlled studies are lacking.

It is conceivable that ongoing research using donor T-cells modified by new suicide genes (e.g. Caspase 9) that my not trigger a host immune response, manipulations of the proteasome or transcription factors (e.g. NFKB), hypomethylating agents or histone deacetylase inhibitors or refined manipulations of other pathways (e.g. mTOR) will change this assessment. However, today, in 2011, I believe we have not succeeded in separating GVHD from GVL effects.

\section{Autologous stem cell transplantation in follicular lymphoma: No \\ Ulrich Dührsen}

University Hospital Essen, Germany

Follicular lymphoma is the most common indolent non-Hodgkin lymphoma. Limited stages may be cured by radiotherapy in about $50 \%$ of cases, while in disseminated stages affecting the majority of patients a palliative approach with an initial watch-and-wait strategy is adopted. Treatment indications include lymphoma-related deterioration of the patient's general condition, constitutional symptoms, local complications and impairment of organ function. High-dose chemo- or chemoradiotherapy with autologous stem cell transplantation may be envisaged in three situations: first-line therapy, treatment of relapse or treatment of transformation which occurs at a frequency of about 3\% per year in follicular lymphoma. Standard first-line treatment of follicular lymphoma currently comprises 6-8 cycles of rituximab-augmented mono- or polychemotherapy followed by two years of rituximab maintenance. Overall response rate (ORR) is on the order of $90 \%$, complete remission (CR) is achieved in about $50 \%$ of patients, and 3-year progression-free survival (PFS) and overall survival (OS) of responders have been reported to be $75 \%$ and $>90 \%$, respectively. Three randomised trials have compared high-dose chemoradiotherapy with conventional therapy in the pre-rituximab era. Two found an extension of
PFS in patients receiving high-dose therapy, but none showed an improvement in OS. Similar results were obtained in a randomized trial comparing high-dose and conventionally dosed chemoimmunotherapy including rituximab. In most studies, therapy-related myelodysplastic syndromes (MDS), acute myeloid leukemias (AML) and solid tumors developed more frequently in patients receiving high-dose therapy than in patients receiving conventional therapy (4-9\% versus $0-3 \%$ after 5 years).

Treatment of relapsed follicular lymphoma is less well defined than first-line therapy. Most commonly, patients are treated with rituximab and a chemotherapy regimen differing from the protocol used in first-line therapy followed by rituximab maintenance. By and large, with this approach ORR is $85 \%$, CR rate is $30 \%$, and 5 -year PFS and OS are $45 \%$ and $70 \%$, respectively. In the pre-rituximab era, a small randomized trial suggested that high-dose therapy with autologous stem cell transplantation is superior to conventional therapy with respect to PFS and OS. This trial, however, received little attention. In the rituximab era, several retrospective studies also came to the conclusion that high-dose chemoimmunotherapy may be superior to conventional treatment. This data, however, must be interpreted with caution, because no information was provided on how patients were selected for the different treatment options. Similar to first-line high-dose therapy, long-term follow-up revealed a significant proportion of patients developing therapy-related MDS and AML.

Patient selection is also a problem in the interpretation of data relating to high-dose therapy in transformed follicular lymphoma. Retrospective data demonstrate 5 -year OS rates of $20-35 \%$ in patients receiving conventionally dosed therapy as compared to rates of $35-60 \%$ in patients undergoing high-dose therapy. The conclusion that high-dose therapy is superior to conventional therapy, however, is invalid because reports on conventional therapy included all patients requiring treatment whereas reports on high-dose chemotherapy were restricted to those able to undergo the procedure. At least one third of patients with transformed follicular lymphoma do not respond to conventionally dosed induction therapy. Since chemosensitivity is considered a prerequisite for high-dose therapy, these patients are generally excluded from retrospective analyses focussing on high-dose approaches.

In conclusion, four randomised trials have failed to show a survival benefit for high-dose therapy with autologous stem cell transplantation when given as first-line therapy for follicular lymphoma, and most showed an increase in potentially lethal long-term complications. Therefore, high-dose chemotherapy cannot be recommended as first-line treatment of follicular lymphoma. In relapsed or transformed disease, no appropriately performed randomised trials are available to assess the value of high-dose therapy. Retrospective data suggest that it may be superior to conventional chemoimmunotherapy, but patient selection may have been responsible for the results reported. Randomised trials are urgently needed to define the place of high-dose therapy with autologous stem cell transplantation in the treatment of patients with relapsed or transformed follicular lymphoma.

\section{T cell manipulation in allogeneic stem cell transplantation} JH Frederik Falkenburg

Department of Hematology, Leiden University Medical Center, The Netherlands

The elimination of malignant hematopoietic cells after allogeneic hematopoietic stem cell transplantation (alloSCT) was initially thought to be attributed to the myeloablative chemotherapy and irradiation administered during the procedure.

Infusion of the hematopoietic stem cell graft was necessary to restore normal hematopoiesis. Therefore, autologous stem cell grafts or grafts derived from identical twins were considered to be the optimal source of hematopoietic stem cells for transplantation because severe immunological complications are associated with 
the use of allogeneic donors including the development of severe graft versus host disease (GVHD).

However, development of GVHD after transplantation is associated with a decreased likelihood of relapse of the malignancy, leading to the concept of graft versus leukemia (GVL) reactivity. Depletion of T cells from the graft reduced the incidence and severity of GVHD, but was associated with an increased risk of relapse illustrating that T cells play a crucial role in the development of both GVHD and GVL. Transplantation with an unmanipulated graft from an identical twin was not associated with a GVL effect illustrating that the mere presence of T cells in the graft was not sufficient to mediate GVL reactivity. Genetic disparity between donor and recipient is required for a significant GVL effect. Clinical studies have illustrated that $T$ cell depletion of the graft leads to a decrease in mortality and morbidity caused by GVHD, but at the cost of higher relapse rates, and therefore $T$ cell depletion as an isolated intervention does not lead to better disease free survival. However, persistent (minimal residual) disease after alloSCT can successfully be treated by donor lymphocyte infusion (DLI), illustrating the potent GVL effect of T cells.

T cell depletion of the graft should be considered the first step in a procedure allowing the application of cellular immunotherapy. Since T cells mediate the GVL reactivity and are responsible for the control of pathogens in the early period post transplant, administration of T cells should be part of the total therapeutic procedure. The main challenge is to separate the GVHD responses from the responses against the malignant cells and the pathogens.

Many clinical and preclinical data have illustrated that these clinical features can be separated. Firstly, $T$ cells recognizing pathogens are not likely to induce GVHD. Several preclinical and clinical data have illustrated that purified T cell populations specific for CMV, EBV, adenovirus and aspergillus can be administered to immunodeficient patients after alloSCT with no risk of developing GVHD. These pathogen specific T cell responses can be purified based on the specific binding of the T cell receptors with peptide/MHC multimers coupled to beads, or based on activation of antigen specific $T$ cells by pathogen specific peptides resulting in gamma interferon production or induction of activation molecules on the cell membrane. Since the memory T cell compartment of healthy individuals mainly consist of pathogen specific $T$ cells, clinical trials have also been initiated to administer only memory $T$ cells with the graft or early after transplantation to allow control of infections in the early post transplant period.

Both GVHD and GVL are caused by T cells recognizing genetic differences between recipient and donor. These genetic differences between individuals comprise (single) nucleotide polymorphisms giving rise to polymorphic proteins resulting in presenting differential peptides in HLA molecules on cells of the recipient and the donor. Following HLA matched alloSCT these so called minor histocompatibility antigens (MiHA) presented in the context of self HLA molecules can be targets for alloresponses of donor T cells. Skewing the T cell response towards MiHA that are selectively expressed on hematopoietic cells of the recipient will result in specific GVL effect with limited GVHD since the malignant cells are part of the hematopoietic system of the recipient. In contrast, MiHA with a broad expression on both hematopoietic and non-hematopoietic cells of the recipient may be targets of T cells causing severe GVHD in addition to the GVL response.

Target antigens broadly expressed on recipient cells that can be recognized by donor $T$ cells will not always give rise to an immune response resulting in severe GVHD. At the time of transplantation the conditioning regimen will cause tissue damage, and patients are at risk for infections which may lead to a pro inflammatory environment. At the time of transplantation, all antigen presenting cells (APC's) capable of inducing a primary T cell response are still of recipient origin, and the lymphopenia shortly after transplantation will lead to an environment where homeostatic proliferation will amplify the immune responses. Professional recipient APC's, the inflammatory environment and homeostatic proliferation may easily induce a primary immune response against a broad variety of MiHA resulting in a high likelihood of GVHD. In contrast, delaying the application of $T$ cells by first performing $T$ cell depletion, and postponed application of DLI may result in in vivo circumstances evoking a more limited T cell immune response, and less susceptibility of target tissues for attack by T cells. Not only the antigen expression and the specificity of the T cells but also the expression of costimulatory and adhesion molecules as well as the level of expression of HLA class I and HLA class II antigens will determine whether or not specific tissues are severely damaged by effector $T$ cells. Postponing DLI can lead to less GVHD, and shift the balance towards GVL reactivity.

Adapting the dose and timing of DLI to the risk of recurrence of the disease can lead to better anti tumor responses with limited GVHD. Even when this two-step procedure may not result in an increased disease free long term survival, the likelihood of developing severe chronic GVHD is lower resulting in a better quality of life.

Not all hematological malignancies are equally capable of inducing a $T$ cell response in vivo, and early relapses after $T$ cell depleted transplantation also warrant the development of new strategies applying in vitro generated T cell responses specific for the hematopoietic cells of the recipient. These strategies include the application of purified hematopoiesis restricted MiHA specific T cell responses isolated by multimere peptide complexes or peptide stimulation similar to the generation and application of pathogens specific $T$ cells. Furthermore, re-engineering $T$ cells by gene transfer of T cell receptor genes or chimeric antigen receptors targeting MiHA or lineage specific antigens will be future applications of tumor specific $T$ cell responses.

In summary, manipulation of a stem cell graft by (partial) removal of $T$ cell populations and the (postponed) administration of selected $T$ cell responses will lead to specific cellular immunotherapeutic strategies resulting in anti pathogen and anti tumor specific reactivities with no or limited GVHD.

Although at present initial T cell depletion with postponed T cell therapy does not lead to an increased disease free survival, overall morbidity due to chronic GVHD may be reduced. The multistep procedure of first depleting $T$ cells combined with administration of specific $T$ cells at defined time points, provides a platform for improving alloSCT in the future.

\section{Is there a place for hematopoietic stem cell transplantation (HSCT) in treating autoimmune diseases: Yes D Farge}

Saint Louis Hospital, Internal Medicine and Vascular Disease Unit, Paris 7 University, France

Chair of Autoimmune Diseases Working Party (ADWP) of the

European Group for Blood and Marrow Transplantation (EBMT)

Autoimmune Diseases (ADs) are a family of more than 100 heterogeneous diseases, which affect 5 to $8 \%$ of the population worldwide, and are characterised by aberrant activation of the immune system with failure of immune regulation to maintain adapted tolerance. Conventional immunosuppression and new biological agents allow disease control in most cases, but are rarely curative and chronically administered immunosuppressive drugs are associated with short and long term significant morbidity and mortality. In addition, severe or rapidly progressive forms of systemic ADs, such as multiple sclerosis (MS), systemic sclerosis (SSc), inflammatory arthritis as rheumatoid arthritis (RA), lupus erythematosus (SLE), or juvenile idiopathic arthritis (JIA), haematological immune cytopenia (HIC) and Crohn's disease resistant to first or second line therapy, require alternative therapeutic strategies. The concept for using HSCT to treat severe AD arose from a large number of experimental data obtained both in genetically prone $A D$ models (lupus and diabetes) and in those obtained after immunisation 
towards a foreign antigen (Acute Experimental Arthritis and Acute experimental Encephalomyelitis) showing the possibility of "cure" with tolerance induction after allogeneic and also after syngeneic (pseudo-autologous) HSCT. In 1996, a first clinical consensus statement for treating severe ADs by HSCT stipulated the basic principles with regard to disease categories, patient selection, mobilisation, in vitro manipulation, conditioning and treatment. Autologous was largely preferred to allogeneic HSCT due to lower risk of severe toxicity. Briefly, patients have been considered for HSCT if: a) with an AD severe enough to have an increased risk of mortality or advanced and irreversible disability; b) the ADs has been unresponsive to first or second line conventional therapy; c) the HSCT can be undertaken before irreversible organ damage, so that significant clinical benefit can be achieved.

Today, more than 2000 patients worldwide have received an HSCT for an AD alone. In the EBMT database, with 1291 patients registered, who underwent 1234 autografts/90 allografts: multiple sclerosis (MS=473), scleroderma ( $(\mathrm{SS}=269)$ and systemic lupus erythematosus $(\mathrm{SLE}=102)$ are the most commonly transplanted diseases, other indications being rheumatoid $(\mathrm{RA}=88)$ or juvenile arthritis $(J \mid A=76)$, autoimmune cytopenia $(n=79)$, vasculitis $(n=37)$ and inflammatory bowel diseases $(n=68)$. Long-lasting responses were obtained in all AD categories with an overall adjusted transplant related mortality (TRM) being $7+3 \%$ at three years, directly related to the type of AD disease, the year of transplant and a center effect related to activity.

In parallel open phase I/II pilot studies in MS, SSC, SLE, Crohn's disease and JIA have shown the feasibility of autologous HSCT and that favourable outcome with a resetting of a dysregulated autoaggressive immune system may occur, rather than total ablation of autoimmune inducing cells. Peripheral Blood Stem Cells (PBSC) are mostly collected, with a prevalent mobilization regimen including the association of Cyclophosphamide (CY) and G-CSF. ADs being extremely heterogeneous, comparison of protocols and outcome depends on careful stratification on diagnosis and phases of diseases. Results of allogeneic HSCT are limited due to small numbers and heterogeneous patient groups and treatment regimens. The mechanism of action has progressively been elucidated. In autologous HSCT, analysis of the regenerating adaptive immune system showed normalization of the restricted T cell repertoire, with sustained shifts in T- and B-cell subpopulations from memory to naïve cell dominance, supportive of thymic reprocessing and re-education of the reconstituting immune system. Restoration of normal or raised levels of $\mathrm{CD} 4+$ regulatory $T$ cells with disappearance of circulating plasmablasts is reported in JIA, and unusual CD8+FoxP3+ regulatory $T$ cell subsets, capable of inhibiting the pathogenic $T$ cell response to autoepitopes in nucleosomes, are seen in SLE following autologous HSCT, but not after conventional immunosuppressive therapies.

All these results were the basis of several prospective phase IIb-II randomised trials both in Europe and United States, to compare HSCT with conventional, approved treatment. Status of the ongoing EBMT approved trials using autologous HSCT to treat severe MS (ASTIMS: HSCT vs Mitoxantrone, now closed for recruitment after $\mathrm{n}=35$ pts included); SSC (ASTIS: HSCT plus ATG and CD34+ selection vs monthly iv Cy $750 \mathrm{mg} / \mathrm{m}^{2}$ for $12 \mathrm{mths}, \mathrm{n}=156 \mathrm{pts}$ included; recruitment completed follow-up ongoing); Crohn's Disease (ASTIC: HSCT vs mobilisation alone, $n=156$ pts included; recruitment ongoing), SLE (ASTIL: HSCT to start soon) and Type 1non ketoacidosic Insulin Dependant Diabetes (ASTID HSCT) illustrate the short and long term benefit that can be obtained with this approach for treating initially severe treated patients.

In 2011, the EBMT AD Working Party (ADWP) has produced new consensus guidelines to harmonise practice and recommendations for both haematologists and autoimmune disease specialists with referrals or who are planning local trials. Where possible prospective clinical trial activity with non-interventional or Phase II-III studies is encouraged, but the rarity of some AD disease may necessitate individual decisions to be taken by multidisciplinary teams. To ensure safety and quality, HSCT in AD should only be performed in European centres accredited by JACIE (or equivalent) with close liaison between HSCT and AD specialists, along with the appropriate facilities and experience and long-term data reporting. Comprehensive cardiopulmonary screening and pre-transplant evaluation of heart, lung, kidney and gastrointestinal function is critically important and patients with advanced end organ disease cardiac disease should be excluded. Among the many conditioning regimens reported, the ADWP recommends cyclophosphamide 200 $\mathrm{mg} / \mathrm{kg}$ with polyclonal or monoclonal anti-T cell serotherapy generally, with cyclophosphamide $120 \mathrm{mg} / \mathrm{kg}$, fludarabine $150 \mathrm{mg} / \mathrm{kg}$ and anti-T cell serotherapy as an alternative in paediatrics and BEAM + anti-T cell serotherapy in multiple sclerosis specifically. Given the high associated TRM in ADs, 'high intensity' or myeloablative conditioning regimens, including irradiation (e.g. TBI) at any dose, should be restricted to clinical trial setting. After HSCT, all patients should remain under the direct routine combined care of the transplant and the AD specialists for at least the first 100 days post transplant, and then on a quarterly basis for the first 2 years even if clinically stable. Thereafter joint annual review as a minimum is recommended. In this context, muticenter collaboration as promoted by the EBMT and the European Orphan Disease Plan, which includes national and regional centers of reference and networks for HSCT in autoimmune diseases, may help with the ongoing follow-up of the promising results brought by HSCT for treating severe AD.

\section{References}

Abrahamsson S, Muraro PA. Immune re-education following autologous hematopoietic stem cell transplantation. Autoimmunity 2008; 41(8): 577-84.

Farge D, Labopin M, Tyndall A et al. Autologous hematopoietic stem cell transplantation for autoimmune diseases: an observational study on 12 years' experience from the European Group for Blood and Marrow Transplantation Working Party on Autoimmune Diseases. Haematologica 2010; 95(2):284-292.

Snowden J, Saccardi R, Allez M et al. on behalf of the EBMT Autoimmune Disease (ADWP) and Paediatric Diseases (PDWP) Working Parties. Haematopoietic stem cell transplantation (HSCT) in severe autoimmune diseases: updated guidelines of the European Group for Blood and Marrow transplantation (EBMT). Bone Marrow Transplant 2011 (in press).

\section{Allogeneic stem cell transplantation in multiple myeloma - Pro Gösta Gahrton}

Department of Medicine Karolinska Institutet, Huddinge

Stockholm, Sweden

Allogeneic stem cell transplantation (allo) is a controversial treatment of multiple myeloma. The ideas behind its use are the same as in other haematological disorders, i.e. to eradicate malignant cells and prevent graft failure by high dose cytotoxic drug or irradiation conditioning and to save the patient from the myeloablative effect of the conditioning by support of normal hematopoietic stem cells. However most important is that immunocompetent cells in the graft should kill malignant cells by a graft versus tumor effect. This effect has been clearly documented in multiple myeloma (GVM). Due to high transplant related mortality (TRM) with myeloablative conditioning reduced intensity conditioning (RIC) has shown feasible and reduces TRM to $12-18 \%$.

The clinical evidence for superior outcome with allo as compared to autologous transplantation (auto) derives mainly from two out of six prospective clinical trials. Only one of these studies indicates 
a tendency for better outcome with auto. Four of them use RIC for allo-conditioning. The studies are based on genetic randomization, i.e. patients with an HLA-identical sibling are offered allo while those who lack a sibling donor receive only auto.

IFM (Intergroup Francais de Myelom) included only "high risk" patients (serum beta-2.microglobulin $>3 \mathrm{mg} / \mathrm{l}$ and deletion of chromosome 13) - 65 received tandem auto-allo and 219 autoauto. RIC consisted of busulfan+fludarabine and high dose ATG. There was no significant difference in event free survival (EFS) but a trend for better overall survival (OS) in the auto-auto group compared to the auto-allo group (median 48 vs 34 months; $p=0.07$ ) based on an intention to treat analysis. If only those patients who actually received the auto-allo transplant (46 patients) or tandem auto transplant (166 patients) were analysed there was still a trend for better OS in the auto-auto group (median 57 vs 41 months; $\mathrm{p}=0.08$ ).

Later the Italian group showed a superior OS for patients who received auto-allo transplantation. 245 patients were included at diagnosis. HLA typing was performed in 162 patients and 80 of these had an HLA-identical sibling donor and 82 had none and comprised the control group. Only 58 patients received auto-allo and 46 auto-auto. Conditioning for allo was $2 \mathrm{GY}$ irradiation. Whether analyzed as an intention to treat or on the actual treatment, there was a significant advantage for the auto-allo group after a followup of 84 month.

The PETHEMA group included only patients who did not enter a complete remission (CR) or a near CR at the first auto. 110 patients had a second transplant, 25 of them allo. RIC consisted of melphalan and fludarabin. Although, there was no significant difference between auto-allo and auto-auto in PFS or OS, there was a trend for better PFS $(p=0.08)$ with the auto-allo approach.

The HOVON Group compared auto-allo and auto followed by maintenance with thalidomide or interferon. The conditioning regimen was TBI 2 GY, Out of 126 patients that had a donor 101 received allo and out of 141 who had not 115 received auto, At an interim analysis (median follow up 38 months) there was no significant difference between the two groups neither in OS or PFS. The 48 months OS was $56 \%$ and $63 \%$ and PFS $39 \%$ and $34 \%$ in the auto-allo and auto-maintenance group respectively.

EBMT has recently published a fifth study that started in 2001. Previously untreated patients received VAD (vincristine+adriamycin+ dexamethasone) or VAD-like induction treatment, and must have had a response status of at least stable disease at the time of inclusion at the first auto. Patients with an HLA-identical sibling then proceeded to allo, while those without received no further treatment or a second auto. 357 patients were included, and median follow-up was 61 months. 108 patients were allocated to the autoallo group (91 actually received the allotransplant according to protocol) and 249 to the auto group. Conditioning was irradiation 2Gy+fludarabin.On an intention to treat analysis at 60 months PFS was $35 \%$ and $18 \%$ (primary end point) and OS $65 \%$ and $58 \%$ in the allo and auto groups respectively. The relapse/progression rate was lower with auto-allo both in patients with and without the del13 chromosomal abnormality. Although the TRM at 24 months was expectedly higher (12\%) in the auto- allo patients the overall long term outcome was superior in this group.

The collaborative BMT CTN 0102 (USA) study included 710 patients (484 auto-auto and 226 auto-allo). RIC conditioning was TBI 2 GY. At 3 years the PFS - the primary end point - was $33 \%$ and $40 \%$, OS $67 \%$ and $59 \%$. Relapse/Proression rate $53 \%$ and $33 \%(p=0.09)$, and treatment-related mortality $8 \%$ and $20 \%(p=0.3)$, in the auto-auto and auto-allo groups respectively. Longer follow up was considered necessary for conclusions.

The conditioning regimen before allo differs in these six studies. Only IFM used high dose ATG. Fludarabin was as well used in the IFM study and also in the studies by PETHEMA and EBMT. The Italian, HOVON and BMT CTN 0102 studies used only 2 Gy TBI without further immunosuppression. Thus none of the studies showing a better outcome or a tendency for better outcome with allo as compared to auto used ATG in the conditioning. The impact of ATG treatment on outcome has been debated. It may be possible that certain ATG types, like the Jurkart T-cell line derived ATG may have a GVHD preventive effect without significant inhibition of GVM while others have an adverse effect on outcome as shown in a retrospective EBMT study.

These studies started before the up-front use of new drugs like bortezomib, thalidomide and lenalidomide, thus the comparison is between allo and auto after a previous auto. In this setting allo seems to be superior at least in two studies, however the higher TRM with allo can not be disregarded. Further studies therefore have to include the new drugs and should preferable include patients with high risk features such as the del17p and $t(4 ; 14)$ chromosomal abnormalities.

\section{Autologous transplantation in follicular lymphoma: Yes}

Pr Christian Gisselbrecht

\section{Hospital Saint Louis, Paris, France}

Follicular lymphoma (FL) is often a disseminated disease with multiple relapses and no long term cure is expected, although very long survival free of disease up to 15 years can be observed. Keeping patient free of disease for several years is one of the main goals of therapy before cure. Before rituximab era the effectiveness of autologous stem cell transplantation (ASCT) in relapse or refractory setting has been reported in phase II studies. The randomized CUP trial, comparing chemotherapy alone to chemotherapy followed by ASCT after three cycles demonstrated a better outcome for both EFS and OS for the ASCT arm.

The broader use of rituximab in combination with chemotherapy was the major advance since 2000. Not only there was an increase in response rate but the addition of maintenance both in first line and at relapse was translated in an improvement of progression free survival and likely survival with a longer follow up. Is the place of ASCT should be dismissed, or can we expect even further improvement to the road of cure. Several published studies give insights of its role in the rituximab era. The first study is the follow up of 2 cohorts of patients treated in 2 prospective randomized trials with the same front line CHVP-IFN treatment. ${ }^{1}$ Rituximab and/or ASCT approaches for relapse or progression were compared. From the 364 patients from the studies, 254 experienced a relapse or progression. Patients who underwent ASCT after salvage rituximab containing regimen had a better OS (95\%) and EFS (67\%) from relapse when compared to $63 \%$ and $46 \%$ respectively for ASCT without rituximab. Nowadays, all patients would receive rituximab in first line treatment; the place of ASCT in relpase has to be evaluated. In the FL 2000 study comparing CHVP-IFN with or without rituximab, 175 patients experienced relapsed/refractory disease. ${ }^{2}$ Treatment was left at the discretion of investigator. ASCT was performed in 42 patients. In multivariate analysis, ASCT and non progression were associated with prolonged OS. For patients $<70$ years the 3 -year OS with ASCT was $92 \%$ versus $59 \%$ for the non transplanted patients. Those results emphasize the role of ASCT for fit enough patients and previously treated with monoclonal anti CD 20 antibody. The efficacy of high dose chemotherapy with ASCT and rituximab has been underlined in the Italian series on $223 \mathrm{FL}$, when they compared the same procedure with or without rituximab with a 5 year OS of $80 \%$ versus $41 \%$ respectively. ${ }^{3}$ Moreover, several studies demonstrated that harvesting stem cell in the presence of rituximab was an effective procedure to collect lymphoma cell free grafts. The EBMT reported a randomized $2 \times 2$ designed trial which evaluated the effects of in vivo purging and/or maintenance with rituximab in patients with relapsed or refractory FL undergoing ASCT with BEAM. ${ }^{4}$

Among the 280 patients, 69 received rituximab in both arms, purging and maintenance; 72 only as in vivo purging without any 
maintenance, 69 as maintenance without in vivo purging and lastly 70 patients did not receive rituximab at all. In vivo purging before ASCT was not associated with better PFS whereas rituximab maintenance yielded higher PFS rate $(50.8 \%$ vs $42.6 \%$ at 5 years $\mathrm{p}=0.02$ ). Five year OS for the whole cohort was $80 \%$ reflecting high salvage success. Improving conditioning regimen with the addition of rituximab before and after ASCT is appealing. Moreover, the incorporation of radioimmunotherapy in the conditioning regimen is safe and warrants randomized study. ${ }^{5}$ Late effects are a concern with ASCT. Data on second malignancies from retrospective studies have shown an increased risk of secondary hematological malignancies, MDS, and solid tumors especially when TBI is used and/or after several lines of treatments. This argues also for not delaying ASCT after multiples relapses and to select drugs for salvage regimens not recognized as damaging stem cells.

The comparison with a cohort of relapsed FL patients treated without ASCT in the EORTC study ${ }^{6}$ provides some information. It appears that for patients treated by $\mathrm{R}$ CHOP and maintenance, the results are quite challenging those previously described with ASCT. However, in this study most of the patients did not receive rituximab for an extensive period upfront. After the PRIMA study, most of the patients would be submitted to a prolonged exposure to rituximab; relapses or refractory patients might have a worse prognosis with an increase risk of rituximab resistance. Whether or not a randomized study is possible in this unsettled issue remains a challenge. New drugs, new antibodies will affect the outcome and the place of reduced intensity conditioning allograft may be discussed with patient as the only way to prevent long term relapses. Nevertheless, to build new strategies we need to better define prognostic factors with new tools such as PET scan and when available biological markers.

\section{References}

1. Sebban C, Brice P, Delarue R et al. Impact of rituximab and/or highdose therapy with autotransplant at time of relapse in patients with follicular lymphoma: a GELA study. J Clin Oncol 2008; 26:3614-3620.

2. Le Gouill S, De Guibert S, Planche L et al. Impact of the use of autologous stem cell transplantation at first relapse both in naive and previously rituximab exposed follicular lymphoma patients treated in the GELA/GOELAMS FL2000 study. Haematologica 2011; 96: 1128-1135.

3. Tarella C, Zanni M, Magni M et al. Rituximab improves the efficacy of high-dose chemotherapy with autograft for high-risk follicular and diffuse large B-cell lymphoma: a multicenter Gruppo Italiano Terapie Innnovative nei linfomi survey. J Clin Oncol 2008; 26: 3166-3175.

4. Pettengell $\mathrm{R}$, Schmitz N, Gisselbrecht $\mathrm{C}$ et al. Rituximab Purging and Maintenance Improves Progression Free Survival but Not Overall Survival In Patients with Relapsed or Resistant Follicular Lymphoma Prior Receiving An Autologous Transplant. Blood (ASH Annual Meeting Abstracts). 2010; 116:absract 3567.

5. Decaudin D, Mounier N, Tilly H et al. (90)Y ibritumomab tiuxetan (Zevalin) combined with BEAM (Z -BEAM) conditioning regimen plus autologous stem cell transplantation in relapsed or refractory low-grade CD20-positive B-cell lymphoma. A GELA phase II prospective study. Clin Lymphoma Myeloma Leuk 2011; 11:212-218.

6. van Oers MH, Klasa R, Marcus RE et al. Rituximab maintenance improves clinical outcome of relapsed/resistant follicular non-Hodgkin lymphoma in patients both with and without rituximab during induction: results of a prospective randomized phase 3 intergroup trial. Blood 2006; 108 3295-3301.

\section{Should cord blood unit be selected according to cell dose or HLA matching?}

Eliane Gluckman', Renato Cunha', Annalisa Ruggeri' Riccardo Saccardi' and Vanderson Rocha'

\section{'Eurocord, Hôpital Saint Louis, Paris, France}

Corresponding Author: Professor Eliane Gluckman, Eurocord office, Hôpital Saint Louis, 1 av Claude Vellefaux, Porte 5, 75010 Paris, France. Tel +33 (0) 1 42499644. E-mail: eliane.gluckman@sls.aphp.fr

\section{Introduction}

The main problem of using umbilical cord blood (UCB) for transplantation is the low number of hematopoietic stem cells available in the UCB unit which translates into increased risk of graft failure, delayed hematopoietic engraftment and delayed immune reconstitution

Different studies highlighted that cell dose and HLA and their complex interaction as the most consistent factors affecting outcome after UCBT.

In the setting of reduced intensity conditioning regimen and double CBT, very few data have addressed the issue of cell dose and number of HLA disparities. The majority of studies that showed that cell dose is an important factor for UCBT outcomes evaluated the impact of TNC on outcomes. Thus, the role of other variables such as CD34+ cell dose, colony forming unit (CFU) and cell viability, is not well established.

In order to investigate whether CD34+ cell dose should be considered as a better surrogate marker for outcomes, Eurocord evaluated two different cohorts of French patients with hematological malignancies receiving a single or double CBT between 1994 and 2010. For all prognostic analysis, prefreezing (pf) and post thawing (pt) total nucleated counts (TNC) and CD34+ cells were divided into 4 categories at $25^{\text {th }}, 50^{\text {th }}$ and $75^{\text {th }}$ percentiles.

In Single UCBT, we analyzed 626 patients. Seventy one per cent of patients had acute leukemia. The majority of patients had either $1(36 \%)$ or $2(49 \%)$ HLA disparities with their graft. Median pf TNC and CD34+ cells were $4.4 \times 10^{7} / \mathrm{kg}$ and $1.6 \times 10^{5} / \mathrm{kg}$. Median pt TNC and CD34+ cells were $3.3 \times 10^{7} / \mathrm{kg}$ and $1.3 \times 10^{5} / \mathrm{kg}$. In multivariate analysis pf and pt TNC and CD34+ were associated with ANC recovery (pf TNC HR=1.29, $p=0.005 ;$ pf CD34+ HR=1.3, $p=0.009$; pt TNC $H R=1.25, p=0.01$, pt $C D 34+H R=1.28, p<0.006)$. $C l$ of acute GVHD at day +100 was $32 \%$ and was not associated with TNC or CD34+ cells count. At 100 days, transplant related mortality (TRM) was $18 \%$. In multivariate analysis pf TNC, pf CD34+ and pt CD34+ were associated with non relapse mortality (NRM) ( $p f$ TNC HR=0.64, $p<0.001$; pf $\mathrm{CD} 34+\mathrm{HR}=0.61, \mathrm{p}=0.003$; pt $\mathrm{CD} 34+\mathrm{HR}=0.61, \mathrm{p}=0.003$ ). Two years disease free survival (DFS) was $40 \%$. This analysis showed the impact of cell dose measured by pf TNC and pf and pt CD34+ on neutrophil recovery and TRM after a single CBT.

In Double UCBT, we evaluated 397 patients. Fifty-seven patients had acute leukemia. The majority of patients had $1(32 \%)$ or $2(61 \%) \mathrm{HLA}$ disparities. Median pf TNC and CD34+ cells were $5.7 \times 10^{7} / \mathrm{kg}$ and $1.7 \times 10^{5} / \mathrm{kg}$. Median pt TNC and CD34+ cells were $3.5 \times 10^{7} / \mathrm{kg}$ and $1.3 \times 10^{5} / \mathrm{kg}$. In univariate analysis, pf TNC, pf CD34 and pt TNC were not associated with ANC recovery. However, there was an association of pt CD34 cell dose and ANC recovery. Autologous recovery was associated with lower $\mathrm{CD} 34+$ cell dose, and it was $49 \%$ in patients receiving $\left(<0.9 \times 10^{5} / \mathrm{kg}\right)$, and $25 \%$ for the remainders $(p<0.001)$. In multivariate analysis, pt CD34 $>0.9 \times 10^{5} / \mathrm{kg}$ was the only independent factor associated with ANC recovery (HR=1.6, $\mathrm{p}=0.001)$. $\mathrm{Cl}$ of acute GvHD at day +100 was $42 \%$ and was not associated with TNC or CD34 cell dose. At 1 year, TRM was $22 \%$, relapse incidence $26 \%$ and 1 year DFS was $50 \pm 3 \%$; none of these outcomes were associated with TNC or CD34 cell dose.

\section{Discussion and conclusion}

There is a general agreement to recommend a prefreezing cell dose $>$ to $3 \times 10^{7} \mathrm{TNC} / \mathrm{kg}$ and $\mathrm{CD} 34+$ cell dose $>1.5 \times 10^{5} / \mathrm{kg}$ but there are many unanswered questions about the standardization of the 
measurement of cell content. Counting CD34+ cells is well standardized in fresh samples. In thawed samples, the lack of internationally validated procedures for dilution, staining and flow-cytometry analyses results in discrepancies between data reported by the cord blood banks and the transplant centers. Transplant units usually draw the sample after washing or diluting the CBU before the infusion, therefore introducing another possible bias. Finally, cells viability assessment is usually carried out through compounds such as 7-ADD, which stains cells in an advanced apoptotic stage. Feasibility and reproducibility of more sensitive methods, such as Annexin-V, are currently under validation. From Eurocord data we calculated that the median loss of TNC after thawing was around $20 \%$ and of CD34+ cells of $10-20 \%$. Clonogenic tests in semi-solid media are poorly standardized; however some authors still propose them as a broader functional assay, representative of the engraftment potential. The situation is even more complex when a double cord blood transplant is used. Most authors say that the stem cell dose of the two units combined should be more than $3 \times 10^{7} \mathrm{TNC} / \mathrm{kg}$.

In summary, we were not able to determine, for each HLA disparity, the best cut-off point for cell dose that is associated with engraftment or survival in patients with malignant disorders. In addition emerging data has indicated that HLA matching at HLA-A, $-\mathrm{C}$ and -B antigens and -DRB1 alleles, rather than high TNC dose, was the more favorable graft characteristic. Therefore, we should give priority to $6 / 6$ UCB units whenever possible. However, the minimum cell dose cutoff for this population is not well established. For $5 / 6$ or $4 / 6$ UCB units, we suggested that the minimum cell dose for all patients with malignant disorders should be higher than $2.5 \times 10^{7} / \mathrm{kg}$ pre-freezing or $2.0 \times 10^{7} / \mathrm{kg}$ post-thawing. For each HLA disparity, the TNC dose should be increased by an increment of 1.5 to $2.5 \times 10^{7} / \mathrm{kg}$. Moreover, matching at locus $\mathrm{C}$, particularly in the case of $6 / 6$ and $5 / 6$ CBU units, should be taken into account. When an adequate single unit is not available, we recommend proceeding with the selection of a second one with no more than $2 \mathrm{HLA}$ disparities between 2 units.

\section{Is there a place for allo SCT in treatment of MM? No position} Hartmut Goldschmidt

\section{Heidelberg University Hospital, Germany}

The results of treatment of multiple myeloma have dramatically improved in the last 15 to 20 years. This is explained both by the introduction of new drugs (Thalidomide, Bortezomib, Lenalidomide) and the advancement of high-dose therapy (HDT) followed by autologous stem cell transplantation. HDT is currently considered as the standard of care as part of up-front therapy for younger ( $<70$ years) patients with multiple myeloma. There is clinical evidence to use tandem-transplantation for patients not achieving a very good clinical response (VGPR) after first HDT followed by autologous stem cell transplantation. Patients with standard risk factors (absence of $t(4 ; 14), t(14 ; 16), 17 p-)$ are projected to live for 7 to 10 years with good quality of life.

Because of the document graft-versus-myeloma (GVM) effect, allogeneic HSCT is an option for treating multiple myeloma. Although treatment with myeloablative conditioning regimes followed by allogeneic HSCT is capable of producing long-term survival, it has not been adopted as standard of care because of unacceptably high rates of transplant-related mortality (TRM) ranging form $30 \%$ to $50 \%$.

$\mathrm{RIC}$ allogeneic HSCT can result in reliable donor engraftment with relatively low TRM compared with myeloablative regimens. However, the GVM effect is not sufficient to achieve sustainable remission without substantial cytoreduction preallografting.

An expert panel of the International Myeloma Working Group (IMWG) concludes that new strategies are needed to make allogeneic HSCT safer and recommend reduced intensity conditioning in the context of clinical trials only. Further clinical studies are urgently warranted to define the role of allogeneic HSCT in Myeloma.
GvHD prevention by T cell modulation. Is photopheresis treatment of choice for CGvHD? Yes Hildegard Greinix

\section{Medical University of Vienna, Austria}

Chronic graft-versus-host disease (cGvHD) is the major determinant of late non-relapse morbidity and mortality after allogeneic hematopoietic stem cell transplantation (HCT). Furthermore, in many patients resolution of cGvHD requires prolonged systemic immunosuppressive treatment. First-line therapy of cGvHD is based on controlled trials and consists of corticosteroids administered with or without a calcineurin inhibitor (CNI). ' However, salvage treatment of corticosteroid-refractory $\mathrm{CGvHD}$ is almost exclusively based on phase II trials or retrospective analyses. ${ }^{2}$ Besides treatment efficacy demonstrated in partial or complete resolution of cGvHD manifestations side-effects of systemic immunosuppressive therapies including severe infectious complications and organ toxicities have to be considered in CGvHD patients in need for salvage treatment. In addition, interactions of certain immunosuppressive agents with comedications need to be taken into account

Extracorporeal photopheresis (ECP) consists of infusion of UVA irradiated autologous peripheral blood mononuclear cells collected by apheresis and incubated with 8-methoxypsoralen. ECP induces apoptosis of leukocytes, and infusion of ECP-treated cells is followed by modulation of cytokine production, reduced stimulation of effector T cells and induction of donor-derived regulatory T cells generating most likely a tolerogenic response to therapy.,4 During the last years ECP has been frequently used as adjunct salvage therapy of corticosteroid-refractory CGvHD achieving consistently high complete responses in up to $80 \%$ of patients with mucocutaneous involvement including sclerodermatous skin manifestations. ${ }^{4-10}$ Furthermore, improvement in visceral manifestations of CGvHD to ECP have been reported by various clinical investigators treating both adult and pediatric patients..$^{4-10} \mathrm{ECP}$ is one of the very few treatment options with proven benefit for patients with lung involvement by CGvHD seen in severe bronchiolitis obliterans syndrome (BOS) ${ }^{8-13}$ Approximately 100 patients with BOS given ECP treatment have been reported achieving a response to ECP in $51 \%$. Fourteen patients reportedly obtained a complete resolution, 20 a partial resolution and 17 an improvement of BOS after ECP therapy. In four studies on patients with steroid-refractory sclerodermatous cGvHD 62 patients were given ECP resulting in overall response in $42 \%$ to $100 \%$ of patients $5,5,9,14$ Of note, response rates were similar in sclerodermatous and lichenoid cutaneous manifestations in three reports ${ }^{5,6}$ whereas Couriel and colleagues achieved higher response rates in sclerodermatous CGvHD compared to lichenoid one. ${ }^{9}$

Besides treatment efficacy a corticosteroid-sparing effect of ECP has been demonstrated by numerous investigators including a report from a prospectively randomized phase II study in 95 patients with corticosteroid-refractory/dependent/intolerantcGvHD patients recently published by Flowers and colleagues. ${ }^{4-10,12}$ Furthermore, significantly improved survival rates and improvements in quality of life have been reported in ECP responders by various clinical investigators. ${ }^{4,5,7,8,9,10}$ Based on currently available evidence ECP has an excellent safety profile both in adult and pediatric patients and does not cause general immunosuppression which is in contrast to many immunosuppressive agents used for treatment of cGvHD. ${ }^{15}$

Thus, no increased rates of infectious complications or recurrence of original malignant disease have been reported after the administration of ECP for treatment of corticosteroid-refractory CGvHD patients. Of note, in an elegant mouse model on acute GvHD Gatza and colleagues observed improved regeneration of both $\mathrm{T}$ cells and $B$ cells after ECP. ${ }^{16}$ Besides the fact that use of ECP requires a venous access that may be difficult in patients with sclerotic cutaneous manifestations or children and adolescents, no other main limitations for administration of ECP to CGvHD patients are currently known. Thus, ECP should be used as second-line therapy of 
corticosteroid-refractory CGvHD patients and could also be a reasonable first choice in certain clinical scenarios. However, data on the efficacy of ECP in first-line treatment of CGvHD are sparse and prospective studies in this area are warranted.

\section{References}

1. Wolff D, Gerbitz A, Ayuk F et al. Consensus conference on clinical practice in chronic graft-versus-host disease (GVHD): first-line and topical treatment of chronic GVHD. Biol Blood Marrow Transplant 2010; 16:1611-28.

2. Wolff $D$, Schleuning $M$, von Harsdorf $S$ et al. Consensus Conference on Clinical Practice in Chronic GVHD: Second-Line Treatment of Chronic Graft-versus-Host Disease. Biol Blood Marrow Transplant 2011; 17:1-17.

3. Knobler R, Barr ML, Couriel DR et al. Extracorporeal photopheresis: past, present, and future. J Am Acad Dermatol 2009; 61:652-65.

4. Greinix HT, Socie G, Bacigalupo A et al. Assessing the potential role of photopheresis in hematopoietic stem cell transplant. Bone Marrow Transplant 2006; 38:265-73.

5. Greinix HT, Volc-Platzer B, Rabitsch W et al. Successful use of extracorporeal photochemotherapy in the treatment of severe acute and chronic graft-versus-host disease. Blood 1998; 92:3098-104.

6. Apisarnthanarax $\mathrm{N}$, Donato $\mathrm{M}$, Korbling $\mathrm{M}$ et al. Extracorporeal photopheresis therapy in the management of steroid-refractory or steroiddependent cutaneous chronic graft-versus-host disease after allogeneic stem cell transplantation: feasibility and results. Bone Marrow Transplant 2003; 31:459-65.

7. Foss FM, DiVenuti GM, Chin K et al. Prospective study of extracorporeal photopheresis in steroid-refractory or steroid-resistant extensive chronic graft-versus-host disease: analysis of response and survival incorporating prognostic factors. Bone Marrow Transplant 2005; 35:1187-93.

8. Messina C, Locatelli F, Lanino E et al. Extracorporeal photochemotherapy for paediatric patients with graft-versus-host disease after haematopoietic stem cell transplantation. Br J Haematol 2003; 122:118-27.

9. Couriel DR, Hosing C, Saliba R et al. Extracorporeal photochemotherapy for the treatment of steroid-resistant chronic GVHD. Blood 2006; 107 3074-80.

10. Flowers ME, Apperley JF, van Besien $\mathrm{K}$ et al. A multicenter prospective phase 2 randomized study of extracorporeal photopheresis for treatment of chronic graft-versus-host disease. Blood 2008; 112:2667-74.

11. Dall'Amico R, Messina C. Extracorporeal photochemotherapy for the treatment of graft-versus-host disease. Ther Apher 2002; 6:296-304.

12. Greinix HT, van Besien $\mathrm{K}$, Elmaagacli $\mathrm{AH}$ et al. Progressive improvement in cutaneous and extracutaneous chronic graft-versus-host disease after a 24-week corse of extracorporeal photopheresis - Results of a crossover randomized study. Biol Blood Marrow Transplant 2011; 17:1775-1782.

13. Lucid CE, Savani BN, Engelhardt BG et al. Extracorporeal photopheresis in patients with refractory bronchiolitis obliterans developing after alloSCT. Bone Marrow Transplant 2011; 46:426-429.

14. Bisaccia E, Palangio M, Gonzalez J: Long-term extracorporeal photochemotherapy in a pediatric patient with refractory sclerodermatous chronic graft-versus-host disease. Transfus Apher Sci 2011; 45:187-190.

15. Suchin KR, Cassin M, Washko R et al. Extracorporeal photochemotherapy does not suppress $\mathrm{T}$ - or B-cell responses to novel or recall antigen. J Am Acad Dermatol 1999; 41:980-986.

16. Gatza E, Rogers CE, Clouthier SG et al. Extracorporeal photopheresis reverses experimental graft-versus-host disease through regulatory $T$ cells. Blood 2008; 112:1515-1521.

\section{Should haplo-SCT be performed with T-cell depletion? Yes Rupert Handgretinger}

Children's University Hospital, University of Tübingen, Germany

Haploidentical stem cell transplantation (SCT) with unmanipulated grafts is associated with a higher risk of Graft-versus-Host
Disease (GvHD) and an intensive posttransplant immunosuppressive therapy is necessary for its prevention. Despite the immunosuppression, GvHD, besides relapse, is still a major cause for therapy failure and contributes to the mortality and short- and long-term morbidity of haploidentical SCT. In addition, chronic GvHD impacts the quality of life after transplantation. A number of various posttransplant pharmacologic regimens have been described, and all of them have their advantages and disadvantages.

In vitro T-cell depletion is the most effective method to prevent GvHD and additionally allows the omission of any posttransplant GvHD prophylaxis, if the number of graft-contaminating T-lymphocytes is low ( $<25.000 / \mathrm{kg}$ recipients body weight). The optimal T-cell depletion methods should result in a high T-cell depletion efficacy and should be associated with a rapid immune reconstitution posttransplant. While earlier methods of T-cell depletion of bone marrow grafts were not very effective, more recently developed methods such as CD34+positive selection or CD3/CD19 depletion allow the effective depletion of T- and B-lymphocytes from mobilised peripheral stem cells (PBSC's). CD34+ positive selection using large-scale magnetic-activated cell sorting results in an indirect depletion of T-cells of approx. 4-5 log. With this method, all nonCD34+ cells, including Natural Killer (NK) cells, dendritic cells and other myeloid cells are discarded. Due to the important role of NK cells for engraftment, methods have been developed for the negative depletion of CD3+ T-lymphocytes (and CD19+ B-lymphocytes) while retaining all CD3/19 -negative cells, including large numbers of NK cells, in the graft. This method is associated with an approx. 10-fold less effective T-cell depletion (3-4 log) and requires in most patients a moderate posttransplant prophylaxis for GvHD. The clinical application of CD3/19 depletion has resulted, especially in the pediatric setting and in some studies in adult patients, in a lower transplant -related mortality (TRM) and accelerated immune reconstitution with a significant lower rate of severe infections compared to the CD34+ positive selection approach. In order to further increase the efficacy of the negative T-cell depletion strategy, methods for the depletion of $\alpha / \beta$ T-lymphocytes via the use of a biotinylated anti- $\alpha / \beta$ antibody followed by anti-biotin labeled magnetic microbeads were developed. Using this strategy, $a / \beta+T-l y m$ phocytes can be depleted with the same efficacy as with CD34+ positive selection (4-5 log) while retaining all $\alpha / \beta$-negative cells, including $\gamma / \delta+$ T-lymphocytes in the graft. A very rapid immune reconstitution was observed after transplantation of haploidentical $\alpha / \beta$ T-cell depleted PBSC's and this method seems to be superior to the previously described methods.

Especially in the setting of haploidentical transplantation, alloreactive Natural Killer (NK) cells are very important in controlling post-transplant residual disease, and patients grafted from NK-alloreactive donors have a lower risk of relapse compared to patients grafted from an NK non-alloreactive donor. This has been shown in adult patients with AML transplanted with CD34+ positively selected cells as well as in children with ALL. However, it has also been shown that T-cell alloreactivity dominates NK cell alloreactivity in minimally T-cell depleted HLA-non-identical pediatric bone marrow transplantation and NK cells will not contribute to posttransplant control of residual disease with concomitant immunosuppressive therapies for the prevention of treatment of GvHD. The most effective antileukemic effects can be obtained in the absence of any posttransplant pharmacologic GvHD prophylaxis, which can only be achieved by an extensive in vitro T-cell depletion of the graft. Therefore, T-cell depletion is necessary to exploit the fully antileukemic activity of donor-derived NK cells and other potential effector cells such as $\gamma / \delta+$ T-lymphocytes after transplantation. In summary, haploidentical SCT should be performed with the best available T-cell depletion method of PBSC's providing an extensive T-cell depletion as well as a rapid immune reconstitution after transplantation. 


\section{Should all MRD positive patients receive allogeneic stem cell transplantation?}

Guenter Henze, Arend von Stackelberg and Cornelia Eckert

Charité - Universitaetsmedizin Berlin, Campus Virchow Klinikum, Dept. of Pediatric Oncology and Hematology, Germany

Measurement of minimal residual disease (MRD) has become an important tool for decision making in childhood acute lymphoblastic leukemia (ALL) during the past years. The aims of determining the residual leukemic cell burden are different depending on the time point of measurement, preceding treatment, the defined cut-off level, the method of measurement, and accordingly, different consequences are drawn from the results.

With conventional methods to assess the risk of relapse it was possible to define prognostic groups with a significantly different prognosis. However, still the largest absolute number of relapses occurred in childhood ALL standard risk groups because the number of "standard risk" patients was overestimated. This has led to the introduction of MRD to assess the risk of relapse more precisely. In Europe, MRD is mostly determined by molecular genetic analyses using immunoglobulin and T-cell receptor rearrangements as clone specific markers. In patients with newly diagnosed ALL as well as with ALL relapse MRD is measured after induction therapy in order to assess the rapidity and the extent of leukemia cell reduction. The sensitivity of molecular genetic measurement is usually in the range of at least $10^{-4}$. According to the definition used in the cooperative AIEOP and BFM trial $2000^{1}$, patients with MRD negativity, i.e., no detectable leukemia any more at 2 defined time points with (minimum required sensitivity $10^{-4}$ ) were classified as standard risk. High risk (HR) patients were defined by MRD levels of $10^{-3}$ or more at both time points. They were qualified for stem cell transplantation (SCT) in $1^{\text {st }}$ complete remission (CR). All others were classified as intermediate risk patients. It could be shown that the prognostication by MRD was indeed improved: the cumulative incidence of relapses was lowest in the standard risk group and highest in the high risk group. 2

In relapse patients MRD measurement was introduced, accordingly, in order to answer the question which patients of the intermediate risk group, mostly patients with late isolated or combined bone marrow relapses, would need stem cell transplantation in $2^{\text {nd }} \mathrm{CR}$. Likewise, it could be shown that rapid early response, i.e., MRD below $10^{-3}$ after induction (day 28-36 after start of therapy), identified a group of patients who had a favourable prognosis with chemotherapy. ${ }^{3}$ Therefore, MRD monitoring was introduced in the relapse trial ALL-REZ BFM 2002, and the predictive value of MRD could be confirmed for rapid early responders; in addition, it could be shown that stem cell transplantation in $2^{\text {nd }} \mathrm{CR}$ was capable of compensating the risk of subsequent relapses in the group of slower responders. ${ }^{4}$

By other groups it was shown that measurement of MRD could also reliably be performed by flow cytometry. Thus, monitoring of MRD early in remission has been shown to be the best instrument to measure the effect of induction therapy and to be the overriding prognostic factor for decision making and allocating patients to a treatment arm with appropriate intensity of therapy.

Another way of using MRD is to get an estimate of residual leukemia prior to SCT. Together with Bader et al. we were able to show that patients with a MRD level higher than the set cut-off of $10^{-4}$ prior to SCT had an inferior outcome than patients with lower MRD levels. ${ }^{5}$ The probability of event-free survival (pEFS) was 0.27 and 0.60 for patients with high compared with low MRD $(p=.004)$.

The informative value of MRD is completely different in both of the described settings. In one setting MRD is used to measure the response to therapy at a high sensitivity in order to make a decision about the following treatment. In contrast, MRD prior to SCT has until now only retrospectively been used to analyse the effect of SCT in patients with different levels of detectable leukemia cells without any further interventional treatment before transplantation aiming at reducing the measured MRD. In both settings, however, the information is consistent that patients with higher levels of MRD have a worse prognosis than patients with lower or no more detectable MRD.

Detectable MRD is a proof of persistent leukemia. Obviously, the treatment performed up to this time point was not capable of eliminating leukemia or at least reducing the leukemic cell burden beyond a measurable threshold. In this sense, detectable MRD indicates non-response or insufficient response to therapy. Recently, we published results on outcome of 93 children and adolescents who had failed to achieve remission after salvage protocol therapy. ${ }^{6}$ The subsequent treatment was given with curative intent including SCT to 51 patients, palliative intent to 23 patients, and 19 children received only "supportive therapy", i.e. no further antileukemic chemotherapy. Median survival times in these three groups were 121,89 and 42 days. The differences in survival were statistically significant $(p<.0001)$ although their relevance may be doubtful. Ten of the 51 patients treated with curative intent died from treatment-related complications and 39 from disease progression. About $70 \%$ of them died in a hospital. Only 2 children are alive and in CR. In contrast, about $70 \%$ of the patients treated without antileukemic therapy passed away at home in their families.

In all of these patients the non-response to therapy was obvious. All of them had microscopically detectable leukemia in bone marrow. However, is it important which method is used to detect leukemia? Does it make a difference whether leukemia is detected by the microscope or by PCR or by flow cytometry? Methods measuring leukemia at the submicroscopic level are clearly more sensitive; but obviously, patients in whom leukemia can be found at a measurable extent have a substantially worse chance for cure compared with MRD negative patients. The fact that even low levels of leukemia predict a poor prognosis is further substantiated by the finding that the detection of submicroscopic bone marrow leukemia at the level of $10^{-4}$ in patients with clinically isolated extramedullary relapse was associated with a significantly worse prognosis compared with patients with lower levels or not detectable leukemia. ${ }^{7}$ In pediatric oncology there is a general agreement that SCT should not be performed in patients who are not in remission. In patients with clinically overt leukemia the prognosis is almost invariably dismal despite SCT. As shown, the prognosis in patients with MRD $>10^{-4}$ prior to $\mathrm{SCT}$ is not absolutely dismal but clearly significantly inferior compared to patients with a lower MRD burden. Thus, the results of SCT in MRD positive patients resemble results of SCT in patients not in remission. The only optimistic expectation is that the residual leukemia might be eliminated or controlled by a graftversus-leukemia effect associated with the potentially occurring graft-versus-host disease. This, however, will not be the case in the majority of patients as shown by the clinically experience, and in addition, the graft-versus-host disease may be deleterious for the patient.

Allogeneic SCT carries a substantial risk for the recipient, even the risk to die from transplant related complications. In addition one has to consider severe adverse late sequelae. Therefore, the decision whether or not to perform a transplant requires balancing of the potential risks and benefits. Ideally, the indication for SCT should be clear and based on solid grounds. Currently we know that the likelihood to fail is high if SCT is being performed in patients not in remission. The decision to do a SCT on the basis: "if we are not able to achieve a remission any technically possible medical intervention is justified" is ethically questionable and because of the hardships posed on the patients in face of the expected failure in disagreement with one of the main principal ethic requirements: nil nocere!

Our answer to the question whether all MRD positive patients should receive allogeneic stem cell transplantation is: No, not all patients and not in any case.

The decision about allogeneic SCT has to take into consideration the disease status, the general condition of the patient, his age, his mental and physical fitness, the family situation and the 
expectations connected with the planned therapy, the treatment course, i.e., the patient's tolerance and the potential complications. The decision must not only be based on the question of MRD positivity or negativity. The aim of any therapy, not only but also SCT, should be cure and not mere eradication of disease.

In summary, SCT is not an emergency break. All of the above mentioned aspects have to be taken into consideration, and the precondition prior to SCT should be optimal. SCT needs a clear indication and not just the hope it might work even if the initial situation does not permit this hope based on available clinical experience. If a clear indication does not exist one should try to work it out based on a controlled clinical trial. There is evidence that in patients with elevated MRD prior to a planned SCT, intervention with targeted therapy may be effective in reducing the level of residual leukemia and create better conditions for success. Allogeneic SCT should have a proven potential benefit for the patient.

\section{References}

1. Flohr T, Schrauder A, Cazzaniga G et al. Minimal residual disease-directed risk stratification using real-time quantitative $P C R$ analysis of immunoglobulin and T-cell receptor gene rearrangements in the international multicenter trial AIEOP-BFM ALL 2000 for childhood acute lymphoblastic leukemia. Leukemia 2008; 31:31.

2. Conter V, Bartram CR, Valsecchi MG et al. Molecular response to treatment redefines all prognostic factors in children and adolescents with B-cell precursor acute lymphoblastic leukemia: results in 3184 patients of the AIEOP-BFM ALL 2000 study. Blood 2010.

3. Eckert C, Biondi A, Seeger K et al. Prognostic value of minimal residual disease in relapsed childhood acute lymphoblastic leukaemia. Lancet 2001; 358:1239-1241.

4. Eckert C, Stackelberg A, Hagedorn N, Barth A, Seeger K, Henze G. Molecular Response to Treatment Predicts Outcome in Isolated, but Not in Combined Bone Marrow Relapses of Childhood Acute Lymphoblastic Leukaemia: Interim Results of Trial ALL-REZ BFM 2002. ASH Annual Meeting Abstracts 2008; 112:2515.

5. Bader $\mathrm{P}$, Kreyenberg H, Henze GH et al. Prognostic value of minimal residual disease quantification before allogeneic stem-cell transplantation in relapsed childhood acute lymphoblastic leukemia: the ALL-REZ BFM Study Group. J Clin Oncol 2009; 27:377-384.

6. von Stackelberg A, Volzke E, Kuhl JS et al. Outcome of children and adolescents with relapsed acute lymphoblastic leukaemia and non-response to salvage protocol therapy: a retrospective analysis of the ALL-REZ BFM Study Group. Eur J Cancer 2011; 47:90-97.
7. Hagedorn N, Acquaviva C, Fronkova E et al. Submicroscopic bone marrow involvement in isolated extramedullary relapses in childhood acute lymphoblastic leukemia: a more precise definition of "isolated" and its possible clinical implications, a collaborative study of the Resistant Disease Committee of the International BFM study group. Blood 2007; 110:4022-4029 (e-pub Aug 2007).

\section{Aspergillus resistance is only seen on the Petri dish but not in the patient! Pro}

$\mathrm{HHof}$

Prof. Dr. med. Herbert Hof, Labor Limbach, Im Breitspiel 15, 69126 Heidelberg/Germany. E-mail: herbert.hof@labor-limbach.de

\section{Problem}

It is not a matter of if but of when resistance of bacteria to antibiotics will develop after widespread use.

Since the clinical use of antifungals for the therapy of systemic fungal infections is increasing and, furthermore, these drugs are now more and more used for empiric therapy (1) and even for prophylaxis $(2,3,4)$, there are growing concerns that resistance among fungi - not only of yeasts (5) but also among aspergilli (6) - will arise. But fungi are different from bacteria (7).

\section{Facts and comments}

In clinical practice the value of in vitro testing of fungi is low. There are 3 major arguments for this opinion defending the statement specified in the title.

1) Do you should wait with the initiation of antifungal therapy until you have the laboratory results of in vitro testing?

There is a general rule in therapy of infectious diseases: Antimicrobial therapy has to be started as early as possible. Retardation of treatment will diminish the outcome (8). This observation holds also true for fungal infections, for example for yeast infections (9)

Thus, one has definitely not to wait until a result from in vitro testing from the laboratory is available, because the results of in vitro-testing of susceptibility will arrive much too late. A calculated, rational therapy has to be initiated as soon as possible.

2) Which test system you prefer? EUCAST or CLSI?

-Testing of susceptibilities of aspergilli to antifungals is not as simple as testing of susceptibilities of bacteria to antibiotics: For scientific, clinical studies either CLSI or EUCAST procedures are followed. These recommendations differ in certain

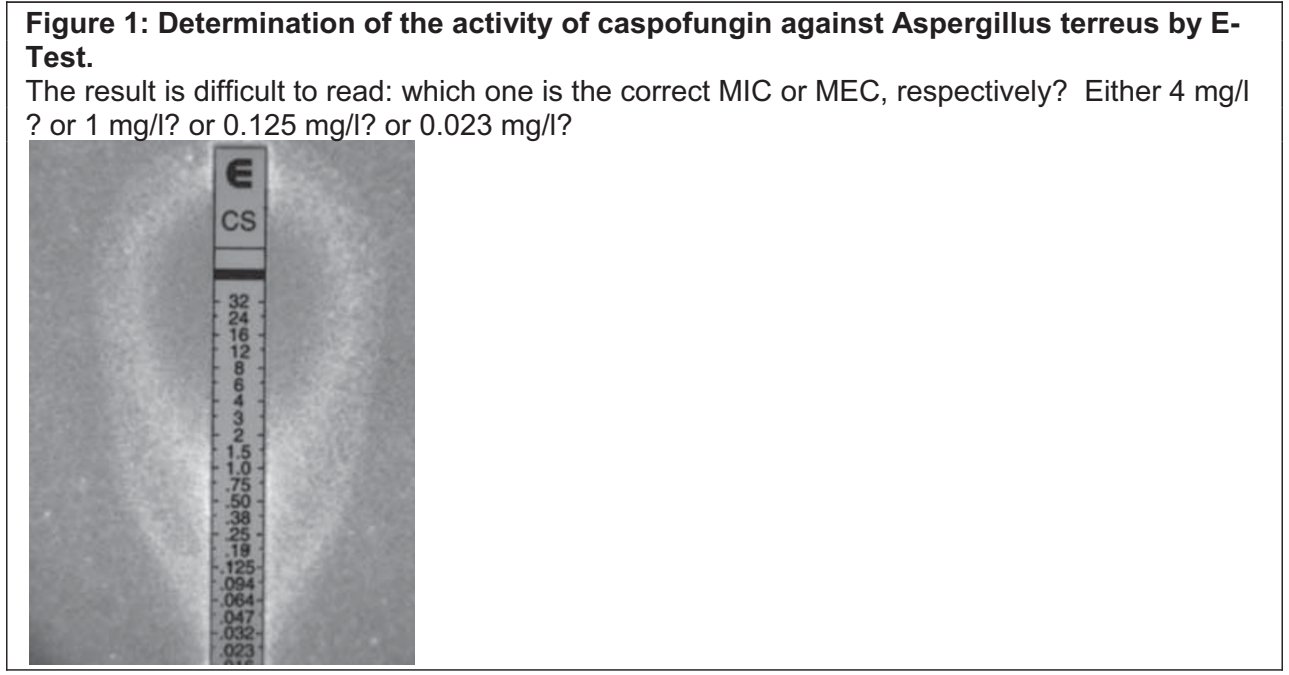


details and the results may not be totally congruent $(10,11)$. For routine testing these microdilution methods of CLSI and EUCAST are too laborious so that other tests such as agardiffusion or E-tests realized on a Petri dish are preferred.

-The reading of the test results is somewhat troublesome. Whereas the fungicidal amphotericin B generally gives a clear cut, visible MIC value, the slowly fungicidal azoles often show a trailing effect which means that there is a gradual inhibition of fungal growth by the increased drug dilutions but no abrupt and total inhibition. For the reading of the activity of the fungistatic echinocandins EUCAST has even generated a new term, namely MEC (minimum effective concentrations), which implies that the endpoint is not sharp. Also the reading of the E-test may pose problems, since sometimes there are two or more inhibition zones visible more or less distinct (Figure 1). Only well-trained personal is able to produce reliable results.

-The interpretation of test results is also difficult. Reliable breakpoints are not available for all antifungals neither in the CLSI nor in the EUCAST standards (10). Such breakpoints are only arbitrarily taken into account for such tests performed on agar plates.

Conclusively, the test methods used in routine laboratory work are suboptimal and hence the assessment of the results is limited. There is a risk that false consequences are drawn from these in vitro tests. An overestimation as well as an underestimation of the activities of antifungals may consequently be deduced from such vague information.
3) Is there a major risk of development of resistance of fungi to antifungals?

It is well known that primary resistance to polyenes or azoles, respectively, may occur in certain Aspergillus spp. (Table 1). The polyenes are widely used in medicine since more than 50 years. There are no tendencies of resistance development among clinical isolates of aspergilli, yet $(1,12)$. Secondary resistance to azoles occurs due to either a mutation in the gene of the target enzyme (13) or by a doubling of gene copies (14), but quite obviously rather rarely and there is no consistent trend of increasing incidence (15).

The susceptibility of a clinical isolate of Aspergillus fumigatus, which is the most frequent and most pathogenic species, can be predicted with a rather high probability (Table 2).

Even after longterm treatment with azoles of patients with haemato-oncologic disorders (16) or lung transplant recipients (17) or with chronic pulmonary diseases (18) including cystic fibrosis (19), there was no increase of incidence of resistant variants.

And indeed, there are several biologic reasons why the problem of resistance among fungi will not increase in future like resistance in bacteria (Table 3). The mycelial form of the aspergilli, which is the only stage found in infected tissues, has only a low tendency to acquire resistance (17). Furthermore, the most relevant resistance mechanism in bacteria, namely the production of enzymes inactivating the drug, for example betalactamases, has never been described in fungi.

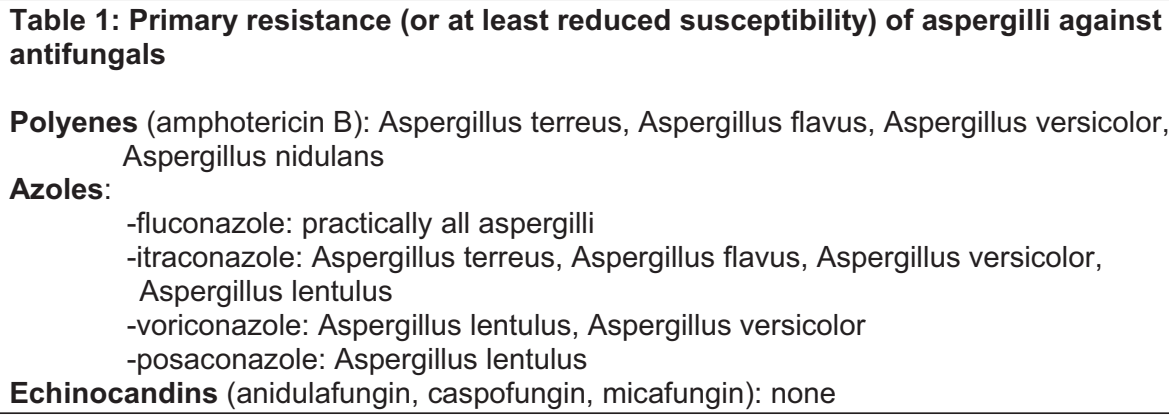

Polyenes (amphotericin B): Aspergillus terreus, Aspergillus flavus, Aspergillus versicolor, Aspergillus nidulans

Azoles:

-fluconazole: practically all aspergilli

-itraconazole: Aspergillus terreus, Aspergillus flavus, Aspergillus versicolor, Aspergillus lentulus -voriconazole: Aspergillus lentulus, Aspergillus versicolor -posaconazole: Aspergillus lentulus

Echinocandins (anidulafungin, caspofungin, micafungin): none

\begin{tabular}{|llll}
\hline \multicolumn{3}{|l}{ Table 2: Expected susceptibility of clinical isolates of Aspergillus fumigatus. } \\
Amphotericin B: & +++ & (about $96 \%)$ & fungicidal \\
Voriconazole: & +++ & (about $99 \%)$ & slowly fungicidal \\
Posaconazole & +++ & (about $99 \%)$ & slowly fungicidal \\
Echinoandins & +++ & (about $98 \%)$ & fungistatic \\
\hline
\end{tabular}

\begin{tabular}{|c|c|}
\hline $\begin{array}{l}\text { Mutations in the target site } \\
\text { which reduce the affinity of } \\
\text { the agents }\end{array}$ & possible \\
\hline $\begin{array}{l}\text { Reduced access of agents possible } \\
\text { i.e. activation of efflux pumps }\end{array}$ & possible \\
\hline $\begin{array}{l}\text { Production of enzymes } \\
\text { which inactivate the drug } \\
\text { before it can bind to the } \\
\text { target }\end{array}$ & inexistent \\
\hline
\end{tabular}


Once a resistance mechanism has evolved in a strain this will most probably lead to a loss of fitness. In case that the selective pressure by an antifungal agent has stopped, those isolates will disappear by the time. Furthermore, those resistance mechanisms having evolved in one strain will not be transferred to other fungi, because transmissible genetic elements, for example transposons or plasmids, baring information for antifungal resistance are not found in fungi (7). Hence, fungi lack that character which is responsible for the explosive expansion of resistance among bacteria.

\section{Conclusion}

In general the in vitro susceptibility of fungi, especially of Aspergillus spp., can be predicted with a high probability. Thus, for routine situations testing is quite dispensible. In any case, an in vitro testing of isolates should not delay the early initiation of therapy. The routine methods for testing in vitro the activities of antifungals, especially of azoles and echinocandins, suffer from inherent practical difficulties and inconsistencies. The interpretations of results may be intricate, because reliable breakpoints are not available for all drugs. In spite of widespread use of polyenes, azoles and echinocandins for therapy, empiric therapy or prophylaxis it can be anticipated that the few resistant strains which exist a priori and may develop under an antifungal regimen, will not expand and distribute this trait to other fungi. Thus, the risk of resistance development of fungi against antifungals seems to be low.

\section{References}

1. Moen MD, Lyseng-Williamson KA, Scott LJ. Liposomal amphotericin B: a review of its use as empirical therapy in febrile neutropenia and in the treatment of invasive fungal infections. Drugs 2009; 69: 361-392.

2. Cornely OA, Böhme A, Buchheidt D et al. Primary prophylaxis of invasive fungal infections in patients with hematologic malignancies. Recommendations of the Infectious Diseases Working Party of the German Society for Haematology and Oncology. Haematologica 2009; 94: 113-122.

3. El Cheikh J, Castagna L, Wang L et al. Once-weekly liposomal amphotericin B for prophylaxis of invasive fungal infection after graft-versus-host disease in allogeneic hematopoietic stem cell transplantation: a comparative retrospective single-center study. Hematol Oncol Stem Cell Ther 2010; 3: 167-173.

4. Bochennek K, Tramsen L, Schedler N et al. Liposomal amphotericin B twice weekly as antifungal prophylaxis in paediatric haematological malignancy patients. Clin Microbiol Infect 2011; e-pub.

5. Vardakas KZ, Samonis G, Michalopoulos A, Soteriades ES, Falagas ME. Antifungal prophylaxis with azoles in high-risk, surgical intensive care unit patients: a meta-analysis of randomized, placebo-controlled trials. Crit Care Med 2006; 34: 1216-1224.

6. Mayr A, Lass-Flörl C. Epidemiology and antifungal resistance in invasive Aspergillosis according to primary disease: review of the literature. Eur J Med Res 2011; 16: 153-157.

7. Hof $\mathrm{H}$. Is there a serious risk of development of resistance to azoles among fungi due to the widespread use and long-term application of azole antifungals in medicine? Drug Resistance Updates 2008; 11: 25-31.

8. Kumar A, Roberts D, Wood KE et al. Duration of hypotension before initiation of effective antimicrobial therapy is the critical determinant of survival in human septic shock. Crit Care Med 2006; 34: 1589-1596.

9. Garey KW, Rege M, Pai MP et al. Time to initiation of fluconazole therapy impacts mortality in patients with candidemia: a multi-institutional study. Clin Infect Dis 2006; 43: 25-31.

10. Lass-Flörl C. In vitro susceptibility testing in Aspergillus species: an update. Future Microbiol 2010; 5: 789-799.

11. Pfaller M, Boyken L, Hollis R et al. Comparison of the broth microdilution methods of the European Committee on Antimicrobial Susceptibility Testing and the Clinical and Laboratory Standards Institute for testing itraconazole, posaconazole, and voriconazole against Aspergillus isolates. J Clin Microbiol 2011; 49: 1110-1112.
12. Moosa MY, Alangaden GJ, Manavathu E, Chandrasekar PH. Resistance to amphotericin $B$ does not emerge during treatment for invasive aspergillosis. J Antimicrob Chemother 2002; 49: 209-213.

13. Snelders E, van der Lee HA, Kuijpers J et al. Emergence of azole resistance in Aspergillus fumigatus and spread of a single resistance mechanism. PLoS Med 2008; 5: e219.

14. Osherov N, Kontoyiannis DP, Romans A, May GS. Resistance to itraconazole in Aspergillus nidulans and Aspergillus fumigatus is conferred by extra copies of the A. nidulans P-450 14alpha-demethylase gene, pdmA. J Antimicrob Chemother 2001; 48: 75-81.

15. Pfaller M, Boyken L, Hollis $R$ et al. Use of epidemiological cutoff values to examine 9-year trends in susceptibility of Aspergillus species to the triazoles. J Clin Microbiol 2011; 49: 586-590.

16. Alanio A, Sitterlé $E$, Liance $M$ et al. Low prevalence of resistance to azoles in Aspergillus fumigatus in a French cohort of patients treated for haematological malignancies. J Antimicrob Chemother 2011; 66: 371-374.

17. Dannaoui E, Meletiadis J, Tortorano AM et al. Susceptibility testing of sequential isolates of Aspergillus fumigatus recovered from treated patients. J Med Microbiol 2004; 53: 129-134.

18. Camuset J, Nunes H, Dombret MC et al. Treatment of chronic pulmonary aspergillosis by voriconazole in nonimmunocompromised patients. Chest 2007; 131: 1435-1441.

19. Amorim A, Guedes-Vaz L, Araujo R. Susceptibility to five antifungals of Aspergillus fumigatus strains isolated from chronically colonised cystic fibrosis patients receiving azole therapy. Int J Antimicrob Agents 2010; 35: 396-399.

\section{Allogeneic ematopoietic Stem Cell Transplantation for older patients ( $>60$ years) with primary or post PV/ET Myelofibrosis \\ Nicolaus Kröger and Haefaa Alchalby}

Department of Stem Cell Transplantation, Hamburg, Germany University Medical Center, Hamburg-Eppendorf

Correspondence: Prof. Dr. med. Nicolaus Kröger, Department of Stem Cell Transplantation, University Medical Center, HamburgEppendorf, Martinistrasse 52, D-20246 Hamburg, Germany, Phone: +49 407410 55864, Fax: +49 40741053795.

E-Mail: nkroeger@uke.uni-hamburg.de

\section{Extended abstract}

Primary myelofibrosis (PMF) is a stem cell-derived clonal myeloproliferative disorder in which the primary disease process is a clonal proliferation of multiple cell elements especially the megakaryocytes. This proliferation is accompanied by an increased secretion of different cytokines with a secondary intramedullary fibrosis, osteosclerosis, angiogenesis, and extramedullary hematopoiesis [1]. Clinically, this disease is characterized with different degrees of cytopenias, hepatosplenomegaly and constitutional symptoms. Myelofibrosis (MF) may occur at advanced stages of polycythemia vera (PV) and essential thrombocythemia (ET) and thus referred to as post-PV and post-ET MF [2]. This disease is challenging for both patients and physicians. The first challenge is the estimation of the potential natural history and life expectancy in every individual patient, since the survival of PMF patients may vary widely from several months to many years [3]. Second, due to the fact that PMF affects primarily the elderly group of patients, it is important as well to estimate the accompanying comorbidities which could influence the therapeutic decisions. Importantly, the pharmacomedical treatment options for this disease such as growth factors, androgens, interferon- $\alpha$ and conventional cytotoxic medications and more recently so-called JAK2 inhibitors lead only to symptomatic palliation without altering the natural history of the disease [4]. Currently, the only available curative therapy for myelofibrosis 
is allogeneic hematopoietic stem cell transplantation which is still associated with a substantial treatment-related morbidity and mortality $[5,6$, 7]. Furthermore, allogeneic stem cell transplantation induces resolution or regression of bone marrow fibrosis in 50\% of OMF patients after myeloablative conditioning between 6 and 12 months post-AHSCT. In the setting of reduced-intensity conditioning one study documented a complete or near complete resolution of bone marrow fibrosis in $59 \%$ of patients by day $100,90 \%$ by day 180 , and $100 \%$ by one year post-transplant $[8,9]$.

The major risk factors in the myeloablative studies were advanced disease status according to Lille-score, mismatched unrelated donors, and also advanced age of the patient.

Reduced-intensity conditioning offers an option to reduce treatment-related mortality (TRM) and leads to a broader HSCT applicablity also to older patients. However, even here in larger studies age was a major prognostic factor for outcome [10]. patient with age $>55$ years had a significant worse outcome than patients $<55$ years ( $5 y$ OS $48 \%$ vs $82 \%, p=0.03$ ) which remained an dependent factor in the multivariate analysis (HR 2.7, $\mathrm{p}=0.02$ ). Different factors may cause the negative impact of age. There was only a trend for a higher non relapse mortality and for higher incidence of relapse for the older patients [10].

A recent analysis of 30 myelofibrosis patients $60-78$ years of age showed a 100 day mortality of $13 \%$ and a 3 year overall and progression-free survival of $45 \%$ and $40 \%$, respectively [11]. Own data in 57 patient with age $>60$ years and advanced myelofibrosis who received after an dose-reduced conditioning regimen stem cell grafts from related or unrelated donors showed a 3 year overall survival of $56 \%$ (unpublished data).

Since the introduction of reduced-intensity conditioning regimens allogeneic stem cell transplantation has become a reasonable curative treatment option also for elderly patients. Despite the risk factor age, elderly patients with advanced disease and no or only few comorbidities can be achieved long term remission (40-60\%) by allogeneic stem cell transplantation $[10,11]$.

However the optimal time point to perform AHSCT for older myelofibrosis patients is not clearly defined. Transplantation is likely to be curative but the possible associated therapy related morbidity and mortality should be balanced against the individual lifeexpectancy without transplantation.

Efficient tools for prediction of disease evolution became urgent which led to the development of several scoring systems such as Lille score [12], Cervantes score [13], International Prognostic scoring system (IPSS) [14], dynamic IPSS (DIPSS) [15]. These models were derived from patient groups treated mostly by a symptom-oriented approach. In advance Lille stage (intermediate or high risk) or IPSS intermediate 2 or high risk the median survival is less than 4 years. On the other hand, performing allogeneic stem cell transplantation when the disease becomes very advanced results in a significantly lower cure chance and an increased TRM. We recommend offering allogeneic stem cell transplantation to intermediate and high risk disease given that the patient is transplant-eligible and a suitable donor is available. Whenever possible HLA-identical siblings are donors of choice for AHSCT, however similar transplant outcome could be achieved with HLA-identical alternative donors using reduced-intensity conditioning [10]. Close monitoring of disease course in low risk patients is mandatory and a sustained change toward more advanced disease should lead to consulting a transplant unit experienced in allogeneic stem cell transplantation for myelofibrosis.

Many significant advances were achieved in the last decade regarding understanding and management of Philadelphia-negative myeloproliferative neoplasms. Much work is still needed at different levels to offer more options to myelofibrosis patients. An important aim is to further reduce toxicity of allogeneic stem cell transplantation and to widen its applicability to older or co-morbid patients as well as to improve post-transplant strategies preferably the prophylactic or preemptive approaches to reduce relapse [16]. Here the increased feasibility of post-transplant strategies through availability of sensitive techniques for MRD detection (such as JAK2 V617F or MPL mutations) $[17,18,19]$ and application of donor lymphocyte infusions may make the prevention and management of relapse more successful. Moreover the availability of JAK2 inhibitors may improve performance status in advanced patients prior transplantation by reducing constitutional symptoms as well as spleen size and therefore facilitate the transplant procedure [20].

\section{References}

1. Tefferi A. Pathogenesis of myelofibrosis with myeloid metaplasia. J Clin Oncol 2005; 23: 8520-8530.

2. Mesa RA, Verstovsek S, Cervantes F, Barosi G, Reilly JT, Dupriez B et al. Primary myelofibrosis (PMF), post polycythemia vera myelofibrosis (postPV MF), post essential thrombocythemia myelofibrosis (post-ET MF), blast phase PMF (PMF-BP): Consensus on terminology by the international working group for myelofibrosis research and treatment (IWG-MRT). Leuk Res 2007; 31: 737-740.

3. Cervantes F, Passamonti F, Barosi G. Life expectancy and prognostic factors in the classic BCR/ABL-negative myeloproliferative disorders. Leukemia 2008; 22: 905-914.

4. Kröger N, Mesa RA. Choosing between stem cell therapy and drugs in myelofibrosis. Leukemia 2008; 22: 474-486.

5. Guardiola P, Anderson JE, Bandini GW, Cervantes F, Runde V, Arcese W et al. Allogeneic stem cell transplantation for agnogenic myeloid metaplasia: a European Group for Blood and Marrow Transplantation, Societe Francaise de Greffe de Moelle, Gruppo Italiano per il Trapianto del Midollo Osseo, and Fred Hutchinson Cancer Research Center Collaborative Study. Blood 1999; 93: 2831-2838.

6. Ballen KK, Shrestha S, Sobocinski KA, Zhang M, Bashey A, Bolwell BJ et al. Outcome of transplantation for myelofibrosis. Biol Blood Marrow Transplant 2010; 16: 358-367.

7. Kerbauy DM, Gooley TA, Sale GE, Flowers ME, Doney KC, Georges GE et al. Hematopoietic cell transplantation as curative therapy for idiopathic myelofibrosis, advanced polycythemia vera, and essential thrombocythemia. Biol Blood Marrow Transplant 2007; 13: 355-365.

8. Thiele J, Kvasnicka HM, Dietrich H, Stein G, Hann M, Kaminski A et al. Dynamics of bone marrow changes in patients with chronic idiopathic myelofibrosis following allogeneic stem cell transplantation. Histol Histopathol 2005; 20: 879-889.

9. Kröger N, Thiele J, Zander A, Schwerdtfeger R, Kobbe G, Bornhäuser M et al. Rapid regression of bone marrow fibrosis after dose-reduced allogeneic stem cell transplantation in patients with primary myelofibrosis. Exp. Hematol 2007; 35: 1719-1722.

10. Kröger N, Holler E, Kobbe G, Bornhäuser M, Schwerdtfeger R, Baurmann $\mathrm{H}$ et al. Allogeneic stem cell transplantation after reduced-intensity conditioning in patients with myelofibrosis: a prospective, multicenter study of the Chronic Leukemia Working Party of the European Group for Blood and Marrow Transplantation (EBMT). Blood 2009; 114: 5264-5270.

11. Samuelson S, Sandmaier BM, Heslop HE, Popat U, Carrum G, Champlin RE et al. Allogeneic haematopoietic cell transplantation for myelofibrosis in 30 patients 60-78 years of age. Br J Haematol 2011; 153: 76-82.

12. Dupriez B, Morel P, Demory JL, Lai Jl, Simon M, Plantier I et al. Prognostic factors in agnogenic myeloid metaplasia: a report on 195 cases with a new scoring system. Blood 1996; 88: 1013-1018.

13. Cervantes F, Barosi G, Demory JL, Reilly J, Guarnone R, Dupriez B et al. Myelofibrosis with myeloid metaplasia in young individuals: disease characteristics, prognostic factors and identification of risk groups. $\mathrm{Br} J$ Haematol 1998; 102: 684-690.

14. Cervantes F, Dupriez B, Pereira A, Passamonti F, Reilly JT, Morra E et al. New prognostic scoring system for primary myelofibrosis based on a study of the International Working Group for Myelofibrosis Research and Treatment. Blood 2009; 113: 2895-901.

15. Passamonto F, Cervantes F, Vannucchi AM, Morra E, Rumi E, Pereira A et al. A dynamic prognostic model to predict survival in primary myelofibrosis: a study by the IWG-MRT (International Working Group for Myeloproliferative Neoplasms Research and Treatment). Blood 2010; 115: 1703-1708. 
16. Kröger N, Alchalby H, Klyuchnikov E, Badbaran A, Hildebrandt $\mathrm{Y}$, Ayuk F et al. JAK2-V617F-triggered preemptive and salvage adoptive immunotherapy with donor lymphocyte infusion in patients with myelofibrosis after allogeneic stem cell transplantation. Blood 2009; 113: 1866-1868.

17. Alchalby $H$, Badbaran A, Bock O, Fehse B, Bacher U, Zander AR et al. Screening and monitoring of MPL W515L mutation with real-time PCR in patients with myelofibrosis undergoing allogeneic stem cell transplantation. Bone Marrow Transplant 2010; 45: 1404-1407.

18. Alchalby H, Badbaran A, Zabelina T, Kobbe G, Hahn J, Wolff D et al. Impact of JAK2V617F mutation status, allele burden, and clearance after allogeneic stem cell transplantation for myelofibrosis. Blood 2010; 116: 3572-3581

19. Kröger N, Badbaran A, Holler E, Hahn J, Kobbe G, Bornhäuser M et al. Monitoring of the JAK2-V617F mutation by highly sensitive quantitative real-time PCR after allogeneic stem cell transplantation in patients with myelofibrosis. Blood 2007; 109: 1316-1321.

20. Pardanani A, Vannucchi AM, Passamonto F, Cervantes F, Barbui T, Tefferi A. JAK inhibitor therapy for myelofibrosis: critical assessment of value and limitations. Leukemia 2011; 25: 218-225.

\section{Mesenchymal stem cells for treatment of graft-versus-host disease \\ Katarina Le Blanc}

Karolinska University Hospital, Stockholm, Sweden

Mesenchymal Stromal Cells (MSCs) are non-hematopoietic progenitor cells found in the bone marrow and many other tissues. In vitro and in vivo, the cells differentiate into adipocytes, chondrocytes and osteocytes after appropriate induction (1).

Both undifferentiated and MSCs induced to differentiate, have immune-modulatory properites and promote peripheral tolerance. In vitro and in vivo in experimental animal models, MSCs suppress alloreactive donor anti-host T-cell responses. MSCs also prevent the maturation of monocytes to first immature dendritic cells (DCs) and next mature myeloid DCs that support T-cell alloresponses. Instead, MSCs re-polarise pro-inflammatory DCs into tolerogenic IL-10+ DCs that together with other effects promote T-cell anergy and Treg induction. Interferon induces MSC to produce indeolamine 2,3 dioxygenase, prostaglandin E2 and other factors that are believed to mediate these effects.

Steroids are the only established treatment for acute GvHD. Mortality is high when the GvHD is unresponsive to steroids. Based on the immunomodulatory properties of MSC s along with the cells' ability to promote repair of injured tissue, it was hypothesized that MSCs may be beneficial in reversing $\mathrm{GvH}$ responses. To date, MSCs have been infused intravenously to several hundred patients with steroid-resistant GvHD (2-13). No acute infusional toxicity has been reported. In a multicenter non-randomized trial of the European Blood and Marrow Transplant Group MSC consortium, 25 pediatric and 30 adults were treated (5). Thirty patients showed a complete response to MSC infusion, 27 of those responded to one single dose of MSCs. The study indicated a trend for better responses in children and their survival was statistically higher. Comparable response rates (58\% complete response) and survival was observed in a pediatric study of 12 patients (12). A median of 8 doses of MSCs were given to each patient. Clinical response was most prominent in patients with GvHD of the gastrointestinal tract. While the previous studies included patients that in the majority of cases had failed (often several) previous GvHD treatments, early MSC therapy, at the time of GvHD diagnosis, has also been attempted (8). Thirty two adult patients were treated with MSCs in combination with corticosteroids for de novo GvHD. Responses were seen in seventy-seven percent of the patients, including $89 \%$ of the patients with gut GvHD. The studies mentioned above exemplify the beneficial effects of MSC infusion in the treatment of acute GvHD. In a recent metaanalysis, Wernicke et al. (13) review a total of 183 patients published to date. The overall response rate is $73 \%$. Pediatric patients have a higher response rate than adults (complete response 57 vs. 45\%). Many questions remain to be answered to optimize MSC treatment. As MSCs are poor stimulators of alloresponses and as GvHD mostly requires prompt initiation of treatment, the majority of patients have received MSCs derived from third-party mismatched donors. However, if and to what degree HLA-matching influences GvHD responses in humans remains unclear. Furthermore, it is well established that MSC are rare cells in vivo and that culture ex vivo is necessary to obtain a sufficient number of cells for a therapeutic effect. However, the influence of culture conditions and media supplements on the efficacy of the cells needs to be established in clinical trials. This is particularly true since no efficacy marker has been established that predicts the clinical outcome of patients treated with MSCs. For example, measurements of MSC-induced lymphocyte suppression in mixed lymphocyte culture does not correlate with clinical response. Trials have used MSCs expanded in the presence of either fetal calf serum or platelet lysate. In vitro properties of MSC expanded in the two media are comparable, but undetected differences may still influence patient responses. So far, data does not indicate an increased risk of relapse or graft rejection when using MSCs. Response rates in the literature indicate that MSCs are a promising tool in the treatment of GvHD.

\section{References}

1. Pittenger MF, Mackay AM, Beck SC, Jaiswal RK, Douglas R, Mosca JD, Moorman MA, Simonetti DW, Craig S, Marshak DR. Multilineage potential of adult human mesenchymal stem cells. Science 1999; 284: 143-7.

2. Le Blanc K, Rasmusson I, Sundberg B, Gotherstrom C, Hassan M, Uzunel M Ringden $O$. Treatment of severe acute graft-versus-host disease with third party haploidentical mesenchymal stem cells. Lancet 2004; 363 : 1439-1441.

3. Fang $B$, Song $Y$, Lin $Q$, Zhang $Y$, Cao $Y$, Zhao RC, Ma Y. Human adipose tissue-derived mesenchymal stromal cells as salvage therapy for treatment of severe refractory acute graft-vs-host disese in two children. Pediatr Transplant 2007; 11:814-817.

4. Fang B, Song $Y$, Liao L, Zhang $Y$, Zhao RC. Favorable response to human adipose tissue-derived mesenchymal stem cells in steroid-refractory acute-versus-host disease. Transplant Proc 2007; 39:3358-3362.

5. Le Blanc K, Frassoni F, Ball L, Locatelli F, Roelofs $H$, Lewis I, Lanino $E$ Sundberg B, Bernardo ME, Remberger M et al. Mesenchymal stem cells for treatment of steroid-resistant, severe acute graft-verus-host disease: a phase II study. Lancet 2008; 371:1579-1586.

6. Muller I, Kordowich S, Holzwarth C, Isensee G, Lang P, Neunhoeffer F, Dominici M, Greil J, Handgretinger R. Application of multipotent mesenchymal stromal cells in pediatric patients following allogeneic stem cell transplantation. Blood Cells Mol Dis 2008; 40:25-32.

7. Von Bohin M, Stolzel F, Goedecke A, Richter K, Wuschek N, Holig K, Platzbecker U, Illmer T, Schaich M, Schetelig J et al. Treatment of refractory acute GvHD with third-party MSC expanded in platelet lysate-containing medium. Bone Marrow Transplant 2009; 43:245-251.

8. Kebriaei P, Isola L, Bahceci E, Holland K, Rowley S, McGuirk J, Devetten M, Jansen J, Herzig R, Schuster M et al. Adult human mesenchymal stem cells added to corticosteroid therapy for the treatment of acute graftversus-host disease. Biol Blood Marrow Transplant 2009; 15:804-811.

9. Lim JH, Lee MH, YI HG, Kim CS, Kim JH, Song SU. Mesenchymal stromal cells for steroid-refractory acute graft-versus-host disease: a report of two cases. Int J Hematol 2010; 92:204-207.

10. Arima N, Nakamura F, Fukunaga A, Hirata H, Machida H, Kouno S, Ohgushi $\mathrm{H}$. Single intra-arterial injection of mesenchymal stromal cells for treatment of steroid-refractory acute graft-versus-host disease: a pilot study. Cytotherpay 2010; 12:265-268.

11. Lucchini G, Introna M, Dander E, Rovelli A, Balduzzi A, Bonanomi S, Salvade A, Capelli C, Belotti D, Gaipa G et al. Platelet-lysate-expanded mesenchymal stromal cells as a salvage therapy for severe resistant graft- 
versus-host disease in pediatric population. Biol Blood Marrow Transplant 2010; 16:1293-1301.

12. Prasad VK, Lucas KG, Kleiner GI, Talano JA, Jacobsohn D, Broadwater G, Monroy R, Kutzberg J. Efficacy and safety of ex vivo cultured adult human mesenchymal stem cells (Prochymal) in pedriatic patients with severe refractory acute graft-versus-host disease in a compassionate use study. Biol Marrow Transplant 2011; 17:534-541.

13. Wernicke C, Grunewald T, Juenger H, Kuci S, Kuci Z, Koehl U, Mueller I, Doering M, Peters C, Lawitschka A, Kolb HJ, Bader P, Burdach S, von Luettichau I. Mesenchymal stromal cells for treatment of steroid-refractory GvHD: a review of the literature and two pediatric cases. Int Archives Med $2011 ; 4: 27$

\section{Should CMV infection post allo HSCT be treated by cell-based therapy? No \\ Per Ljungman}

Dept of Hematology, Karolinska University Hospital and Section of Hematology, Division of Medicine Huddinge, Karolinska Institutet, Stockholm, Sweden

Cytomegalovirus infection remains an important clinical problem after allogeneic HSCT. New diagnostic techniques allowing the effective use of preemptive therapy have significantly decreased the rate of CMV disease and also of CMV associated mortality. The most commonly used strategy is weekly monitoring with quantitative PCR detecting CMV DNA. The best level of viral load to initiate antiviral therapy has not been clearly defined and it is likely that it varies depending on the patient's risk for developing CMV disease. It has been shown that ganciclovir, valganciclovir, and foscarnet can be used with similar efficacy for controlling CMV replication. Although these antiviral drugs are associated with significant side effects, therapy can be given effectively and safely in the majority of patients. Second reactivations are quite common but can also in most patients be controlled by a second course of antiviral therapy. This results in a rather small cohort of patients, who develop significant problems caused by CMV or anti-CMV therapy. Resistance to antiviral drugs exists but is still despite two decades of widespread use of the available antiviral agents still uncommon. At least three new antiviral drugs are also in development preliminary showing promising safety can efficacy characteristics. These drugs have potential both for prophylaxis against and therapy of CMV infection. Another interesting development is the promising results from a phase II study of a CMV vaccine showing not only the capacity of the vaccine to induce CMV-specific immunity but also a reduction in CMV viral load. Another CMV vaccine has shown some efficacy in a phase II study of solid organ transplant recipients. Phase III studies are in the planning stages.

Specific T-cell therapy against CMV has been in development for 25 years and a lot has been learned about CMV immunology and CMV specific immune reconstitution through these phase I and II studies. No phase III study has yet been performed comparing T-cell therapy to standard approaches. Furthermore, it has been clearly shown that long term control can only be achieved by reconstitution of CMV specific immunity and this can be achieved by CMV-specific T-cell therapy. Unfortunately, we still do not have a cell based therapy product that can be implemented in routine use. Therefore, cell based therapy is likely to remain a solution for a small subgroup of patients failing the routine strategy of monitoring and preemptive therapy or for patients with high risk characteristics for development of CMV associated problems such as patients undergoing HSCT from haplo-identical or other mismatched donors. Thus, still today standard antiviral approaches with currently available drugs remain the main option for CMV management.

\section{GVHD prevention: Pro alemtuzumab}

Stephen Mackinnon

Department of Haematology, University College London, Pond Street, London NW3 2QG, Tel: 44207830 2301, Fax: 44207830

2092. E-mail: s.mackinnon@ucl.ac.uk

The antibody and pharmacokinetics

Alemtuzumab (CAMPATH-1H) is a humanised lgG1 monoclonal antibody directed against the CD52 antigen, which is widely expressed on all human lymphoid cells except terminally differentiated plasma cells. Delayed clearance of the monoclonal antibody may impair immune reconstitution, affect rates of viral reactivation and limit efficacy of the donor T cell mediated GVL effect, either derived from the graft itself, early adoptive immunotherapy or later DLI. By administering the antibody to the recipient as part of the conditioning regimen, it will result in T cell, B cell and dendritic cell (DC) depletion of the recipient. If sufficient antibody is circulating on the day of transplantation, this will result in T cell depletion of the graft, thereby potentially reducing the incidence and severity of GVHD. The half-life of alemtuzumab in humans is dependent on the amount of target CD52 antigen in the patient. Following an in vivo dose of $20 \mathrm{mg} /$ day for 5 days $(-8$ to -4$)$ prior to allogeneic transplantation there is persistence of alemtuzumab in vivo past day 0 sufficient to cause T cell lysis by complement fixation and ADCC. Using this dose schedule, significant levels of antibody persist through day +28 post-transplant.

\section{Reduced intensity regimens incorporating alemtuzumab}

The most commonly used regimen combine alemtuzumab with fludarabine and an alkylating agent, usually melphalan or busulfan. Alemtuzumab has also been added to the BEAM regimen and used in reduced intensity conditioning for lymphoma.

\section{Chimerism}

The largest experience has used alemtuzumab in combination with fludarabine and melphalan. Three patterns of chimerism have been documented using this regimen.

1. Fully donor in all lineages.

2. Mixed chimera in all lineages.

3. Fully donor myeloid chimerism with mixed T cell chimerism.

As this approach to transplantation is heavily dependent on graftversus-leukemia (GVL) or graft-versus-tumor effects, the development of mixed $T$ cell chimerism following reduced intensity stem cell transplantation could be associated with a higher incidence of disease relapse. Therefore, if mixed chimerism persists once immunosuppression has been discontinued, attempts to promote full donor chimerism and GVL activity using donor leukocyte infusion reduce disease recurrence. Most patients with stable mixed chimerism will achieve full donor chimerism following the administration on donor leukocyte infusions.

\section{GVHD}

Perhaps the most impressive effect of alemtuzumab as part of a reduced intensity conditioning regimen is in the prevention of GVHD. Published results of sibling donor SCT using other nonmyeloablative conditioning regimens have shown a $38 \%$ to $60 \%$ incidence of grade II-IV acute GVHD that is the primary cause of death in some patients. However, when alemtuzumab has been used as part of the conditioning regimen using HLA identical siblings, most patients do not develop any GVHD and the reported incidence of acute grade II - IV GVHD following HLA identical sibling transplantation was $5 \%$. When transplants using unrelated donors are assessed, the effects of alemtuzumab in not only preventing GVHD but also in limiting transplant-related mortality are particularly impressive. For reduced intensity regimens that do not include alemtuzumab, the reported experience of unrelated donor SCTs using a fludarabine + melphalan protocol, observed high rates of severe GVHD, with 1 in 4 patients dying directly as a result of GVHD. In contrast, a similar regimen containing alemtuzumab was associated with a low incidence of GVHD despite significant HLA disparity in many of 
the transplants. Only $6 \%$ of patients had grade III - IV acute GVHD and only $15 \%$ developed grade II acute GVHD.

\section{Disease-specific Outcomes}

Acute Myeloid Leukemia. We have recently reported the pre- and post-transplant factors determining overall survival in 168 patients with acute myeloid leukemia transplanted using an alemtuzumab based reduced intensity conditioning regimen with a median duration of follow-up of 37 months. The 3-year overall survival for patients transplanted in CR1 or CR2/CR3 was $50 \%$ and $44 \%$, respectively compared to $15 \%$ for patients with relapsed/refractory disease. Increased exposure to cyclosporine A in the first 21 days post-transplant was associated with an increased relapse risk and decreased overall survival. These data confirm the presence of a potent graft-versus-leukemia effect after a T-cell depleted reduced intensity conditioning allograft in acute myeloid leukemia and identify CsA exposure as a manipulable determinant of outcome in this setting.

Hodgkin's Lymphoma. The therapeutic options for patients with Hodgkin's disease who relapse soon after completion of first line chemotherapy or have failed an autologous transplant are very limited. We recently reported the use of alemtuzumabbased reduced intensity conditioning in 76 patients (42 sibling, 34 unrelated donors) with Hodgkin's lymphoma. At transplant 17 patients were in CR, 32 in PR, 26 refractory and 1 untested. They had failed a median of 5 lines of therapy and 45 had relapsed following an autograft. DLI were given to 22 patients for mixed chimerism and to 24 for relapse. Nineteen of the 22 patients given DLI for mixed chimerism converted to full donor chimerism and only one of these patients subsequently relapsed. Nineteen of the 24 patients given DLI for relapse responded (14 CRs + 5 PRs). The current progression free survival for all 76 patients was $59 \%$ at 4 years.

Non-Hodgkin's Lymphoma. Results in 130 patients with NHL who received transplantation using alemtuzumab, fludarabine and melphalan regimen were recently reported. Forty eight had aggressive $\mathrm{NHL}$ and 82 had follicular lymphoma. In the patients with aggressive lymphoma the nonrelapse mortality was $32 \%$ with a progression free survival of $54 \%$ in patients with chemosensitive disease. In contrast, the patients with follicular lymphoma had a lower nonrelapse mortality with the same regimen of $8 \%$ in sibling and $22 \%$ in unrelated donor transplants. Although relapses occurred in patients transplanted for follicular lymphoma, most patients had durable CRs following DLI resulting in a 4 year current progression free survival of $90 \%$ for sibling and $64 \%$ for unrelated donor transplants.

\section{Summary}

Alemtuzumab reduces the incidence of acute and chronic GVHD and reduces GVHD-related mortality. For some diseases such as acute leukemia, NHL and Hodgkin lymphoma equivalent or superior survival can be achieved when compared to $T$ cell replete regimens with improved quality of life associated with the reduction in chronic GVHD.

\section{Can Treg Prevent GvHD without losing GvL? Yes}

Massimo F Martelli, Franca Falzetti, Alessandra Carotti, Mauro Di lanni, Loredana Ruggeri, Adelmo Terenzi, Antonio Pierini, Andrea Velardi and Franco Aversa

Perugia University Ospedale, Perugia, Italy

In HLA- haploidentical 2-3 loci mismatched haematopoietic stem cell transplantation (HSCT) for acute leukaemia a TBI-based conditioning followed by a megadose of highly purified CD34+ cells ensures a high engraftment rate with negligible GvHD and over 40\% event-free survival (EFS) (Aversa et al., NEJM 1998; Aversa et al., JCO 2005). In patients with acute myeloid leukaemia (AML) who were transplanted in any remission from donors who were able to exert donor-vs-recipient natural killer (NK) cell alloreactivity a powerful GvL effect raised the EFS rate to over $60 \%$. (Ruggeri et al., Science 2002; Blood 2007; Stern et al., Blood 2008; updated 2011). A major drawback is that extensive ex and in vivo T cell depletion delays recovery of immune responses, leading to a high incidence of life-threatening infections.

Various strateges have been proposed to hasten post-transplant immune rebuilding. Studies in murine moodels of HSCT across major MHC barriers showed that CD4+ CD25+ T regulatory cells (Tregs) suppressed lethal GvHD and favoured post-transplant immune reconstitution when co-infused with conventional T cells (Tcons). In a recent clinical study (Di lanni $\mathrm{M}$ et al. Blood 2011) we reported that adoptive transfer of freshly isolated natually occurring Tregs, followed by a Tcon infusion, prevented GvHD in the absence of any post-transplant immunosuppression, promoted lymphoid reconstitution and improved immunity to opportunistic pathogens. This first clinical study combining donor Tregs and Tcons in the setting of haploidentical HSCT provided proof of principle that Tregs are a conserved mechanism in humans and demonstrated that in vitro expansion of Tregs (polyclonal Tregs or recipient-type specific Tregs) is not an indispensable step when designing Tregbased cellular therapies.

Since the strategy was not, unfortunately, associated with a reduction in transplant related mortality (TRM) a new protocol was designed to reduce the conditioning-related extra-haematological toxicity and TRM. In the conditioning regimen alemtuzumab was administered instead of cyclophosphamide 16 days before TBI and 20 days before the Treg infusion so as to prevent it from interfering with Tregs and Tcons. We also modified the Treg selection procedure to raise the percentage of FoxP3 positive cells from $70 \%$ to approximately $90 \%$ in the final fraction.

The figure below illustrates the protocol.

So far, we have transplanted 18 consecutive patients with acute leukaemia at high risk of relapse, who had a median age of 43 years (range 23-61). They received a median of $8.9 \times 10^{6}$ (range 8.1-10.5) CD34+, 2.9 $\times 10^{6}$ (range 1.6-4.8) Tregs and $0.9 \times 10^{6}$ (range 0.5-3) Tcons. At present we have a median follow-up of 5 months (range 2-14). All patients sustained full donor-type engraftment. Only 3 patients developed GVHD which responded propmptly to post-transplant cyclosporine and steroid therapy in the first 2, who have by now been off-therapy for several months. The third is still being treated. Two patients have died of non-leukaemic causes.

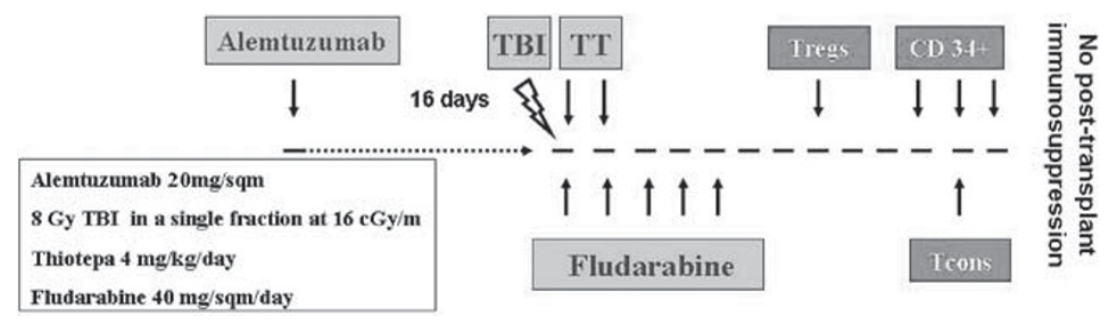


The Table reports details of all 18 patients and outcomes.

\begin{tabular}{|c|c|c|c|c|c|c|c|c|}
\hline NAME & AGE & $\begin{array}{l}\text { DISEASE and } \\
\text { STATUS at BMT }\end{array}$ & RISK FACTORS & NK allo & $\begin{array}{c}\text { ENGRAFT } \\
\text { MENT }\end{array}$ & GVHD & RELAPSE & PRESENT STATUS \\
\hline PT & 45 & $\mathrm{AML}-2^{\circ} \mathrm{CR}$ & Secondary to ALL & YES & +13 & NO & NO & Alive and Well $(+435)$ \\
\hline SE & 33 & $\mathrm{AML}-1^{\circ} \mathrm{CR}$ & FLT3-ITD,$+ t(6 ; 9)$, PIF & NO & +28 & NO & NO & Alive and Well $(+404)$ \\
\hline $\mathrm{RC}$ & 22 & $\mathrm{AML}-2^{\circ} \mathrm{CR}$ & Relapse after consolidation & YES & +11 & YES & NO & Alive and Well $(+330)$ \\
\hline GL & 37 & PDC Lymph - PR & Partial remission & NO & +20 & NO & NO & Alive and Well (+299) \\
\hline $\mathrm{PC}$ & 32 & $\mathrm{AML}-1^{\circ} \mathrm{CR}$ & PIF & NO & +12 & NO & NO & Alive and Well $(+292)$ \\
\hline SC & 45 & $\mathrm{AML}-2^{\circ} \mathrm{CR}$ & FLT3-ITD+, compex karyotype & NO & +12 & YES & NO & Alive and Well $(+265)$ \\
\hline $\mathrm{CS}$ & 21 & $\mathrm{AML}-1^{\circ} \mathrm{CR}$ & $t(16 ; 16),-7$, cytogenetic relapse & No & +15 & NO & NO & Alive and Well (+257) \\
\hline VM & 52 & ALL $-1^{\circ} \mathrm{CR}$ & $\mathrm{t}(3 ; 7 ; 14), \mathrm{TCR}+$ & NO & +12 & NO & NO & Dead - Fulminant Hepatitis $(+17)$ \\
\hline $\mathrm{PF}$ & 23 & Biph $\mathrm{AL}-1^{\circ} \mathrm{CR}$ & Complex karyotype & NO & +15 & NO & NO & Alive and Well $(+191)$ \\
\hline PM & 46 & $\mathrm{AML}-1^{\circ} \mathrm{CR}$ & FLT3-ITD,+ NPMc $^{+}$ & NO & +10 & NO & NO & Alive and Well $(+158)$ \\
\hline SC & 60 & $\mathrm{AML}-1^{\circ} \mathrm{CR}$ & Complex karyotype, PIF & YES & +12 & NO & NO & Alive and Well $(+145)$ \\
\hline VM & 35 & $\mathrm{AML}-1^{\circ} \mathrm{CR}$ & dell1q & NO & +16 & NO & NO & Alive and Well $(+124)$ \\
\hline NR & 46 & $\mathrm{AML}-1^{\circ} \mathrm{CR}$ & FLT-ITD + , meningeal infiltration & NO & +10 & YES & NO & Alive with GvHD $(+110)$ \\
\hline$L D$ & 40 & $\mathrm{AML}-1^{\circ} \mathrm{CR}$ & FLT-ITD,+ NPMc + , PIF & NO & +11 & NO & YES $(+77)$ & Dead in relapse $(+95)$ \\
\hline PL & 50 & $\mathrm{AML}-1^{\circ} \mathrm{CR}$ & FLT3-ITD-, NPMc- & NO & +13 & NO & NO & Alive and Well $(+82)$ \\
\hline TR & 31 & $\mathrm{AML}-1^{\circ} \mathrm{CR}$ & Secondary to MDS & YES & +13 & NO & NO & Alive and Well $(+75)$ \\
\hline $\mathrm{BC}$ & 53 & $\mathrm{AML}-1^{\circ} \mathrm{CR}$ & NPMct, cutaneous relapse & YES & +11 & NO & NO & Alive and Well $(+61)$ \\
\hline MA & 61 & $\mathrm{ALL}-1^{\circ} \mathrm{CR}$ & $\mathrm{Ph}+$ & YES & +9 & NO & NO & Dead - Pneumonia $(+14)$ \\
\hline
\end{tabular}

These preliminary results confirm the low incidence of GvHD, show the modified conditioning regimen is associated, as expected, with mild extra-haematological toxicity and indicate that rebuilding immunity with natural CD4+CD25+FoxP3+ Treg and Tcon adoptive immunotherapy is associated with a low incidence of TRM.

The crucial question is whether the GvL effect is maintained because, since FOXP3 Tregs are indispensable for maintaining dominant self tolerance and immune homeostasis, they can suppress antitumor immune responses and favour tumor progression. Even though data need to be confirmed in a much larger series of patients over a longer follow-up period, impressively only one patient has relapsed out of all those transplanted in remission in both of our clinical trials with Treg and Tcon immunotherapy. Robust post-transplant generation of donor vs recipient alloreactive NK cell clones probably contributed to prevent relapse in about $50 \%$ of AML patients who received transplants from potential NK alloreactive donors. In the others the GvL effect might have been mediated by high numbers of $T$ cells whose action was exerted in the absence of any post-transplant immunosuppression. What clearly emerges is that the Treg infusion does not appear to weaken the GvL effect.

\section{Combined antifungal therapy is not more efficient than single agent therapy Georg Maschmeyer}

Professor of Internal Medicine, Chair, Dept. of Hematology, Oncology and Palliative Care

Director, Center for Hematology, Oncology and Radiotherapy, Klinikum Ernst von Bergmann

Academic Teaching Hospital of Charité University Medical School Berlin, Charlottenstr. 72, D-14467 Potsdam, Germany, Phone: +49 331241 6001, Fax: +49 331241 6000. E-mail: gmaschmeyer@ klinikumevb.de

Invasive fungal infections are associated with severe morbidity and high mortality rates. For invasive cryptococcosis, the combination of an amphotericin B preparation with flucytosine is an accepted gold standard, backed-up by appropriate, prospective clinical studies. The standard antifungal treatment of invasive candidiasis, aspergillosis and zygomycosis is systemic monotherapy. For aspergillosis, voriconazole and liposomal amphotericin B are first choice, while for candidiasis in clinically unstable patients, an echinocandin is preferred. In case of uncomplicated invasive candidiasis, fluconazole monotherapy may be preferred, unless the patient has been pretreated with an azole. Zygomycosis requires a combination of surgical debridement and systemic amphotericin $B$, with posaconazole for salvage treatment. Attempts to combine fluconazole with amphotericin B for invasive candidiasis have shown only little clinical benefit and are not regarded as a valid treatment standard. The combination of voriconazole or amphotericin B with an echinocandin shows promising preclinical results, but has not yet been proven to improve clinical outcome in appropriate studies. The ongoing randomized trial on voriconazole with or without anidulafungin for primary treatment of invasive aspergillosis will be a landmark study to decide on a potential clinical benefit of such a combined antifungal treatment approach. Before results are valid, however, monotherapy remains the treatment of first choice for patients with invasive fungal infections, apart from cryptococcosis. 


\author{
Aspergillus resistance is seen on the petri dish and in the \\ patient \\ Georg Maschmeyer \\ Professor of Internal Medicine, Chair Dept. of Hematology, \\ Oncology and Palliative Care \\ Director, Center for Hematology, Oncology and Radiotherapy, \\ Klinikum Ernst von Bergmann \\ Academic Teaching Hospital of Charité University Medical School \\ Berlin, Charlottenstr. 72, D-14467 Potsdam, Germany, \\ Phone: +49 331241 6001, Fax: +493312416000. \\ E-mail: gmaschmeyer@klinikumevb.de
}

The most recently approved triazole antifungals, voriconazole and posaconazole, have become clinical treatment standards for firstline treatment of invasive aspergillosis (voriconazole) and systemic antifungal prophylaxis in acute myeloid leukemia patients undergoing remission induction and in allogeneic stem cell transplant recipients with significant graft-versus-host disease (posaconazole) since the publication of landmark studies in 2002 and in 2007. Almost unknown to the medical community, tons of modern triazole antifungals are used in agriculture. First alarming data from mycological laboratories indicated an increasing number of Aspergillus fumigatus isolates resistant to itraconazole, voriconazole and posaconazole since 2007 (Verweij et al., N Engl J Med 2007; Howard et al., Emerg Infect Dis 2009). This trend has been on the rise since these early reports, and obviously multi-azole resistant A.fumigatus isolates have now been identified not only in the Netherlands and in UK, but also in China, Brazil, Portugal and the Czech Republic (Lockhart et al, Antimicrob Agents Chemother 2011). While from a microbiological perspective, this phenomenon might not yet be alarming, clinical observations on fatal invasive Aspergillus infections caused by multi-azole resistant A.fumigatus strains indicate that there is indeed a clinically relevant development towards life-threatening mold infections no longer manageable with triazole antifungals (van der Linden et al., Clin Infect Dis 2009). As yet we do not definitively know if resistance emerges, or resistant strains are selected, under azole treatment in patients (Howard et al, Emerg Infect Dis 2009), or if multi-azole resistant A.fumigatus are acquired from multiple environmental sources (Verweij et al, Lancet Infect Dis 2009). Strict limitation of clinical use of broad-spectrum azole antifungals to high-patients according to evidence-based guidelines is warranted. At the same time, (mis)use of broad-spectrum azoles in agriculture must be critically reconsidered.

\section{Donor Selection To Prevent Relapse: According to KIR typing Jeffrey S Miller}

University of Minnesota Cancer Center, Minneapolis, Minnesota, USA, 55455

Natural killer (NK) cells can mediate potent anti-tumor and infection protection responses by direct cytotoxicity and cytokine production and thus they play an important role in the innate immune response. Their effector function is governed by a complex balance of activating and inhibitory signals transferred via several classes of receptors, a number of which recognize "self" MHC class I antigens. Self-tolerance is mediated by inhibitory killer immunoglobulin-like (KIR) that transmit signals that interrupt the cytolytic pathway upon binding of their cognate class I HLA ligands. The loss of KIR-ligand expression by infected or malignant targets renders them susceptible to NK cell lysis, particularly when the targets also present ligands for activating receptors. KIR are also a major determinant for NK cell education or licensing, the process by which NK cells acquire function through interaction of KIR and cognate self ligands. We have shown that KIR are particularly important for educating interferongamma responses to tumor targets while degranulation function (CD107a expression) can be educated through KIR and NKG2A (which recognizes non-classical HLA-E as its ligand). Simply stated,
KIR expressing NK cells are functionally hyporesponsive if they never encounter "self". Several clinical strategies have been developed using alloreactive NK cells for therapeutic benefit using adoptive transfer or hematopoietic cell transplantation (HCT). In both settings, NK cell alloreactivity have been determined in an HLAmismatched haploidentical setting using pioneering predictive models initially described by Velardi and colleagues, who showed a benefit to KIR-ligand mismatching (in the GVHD direction for HLA-B and HLA-C KIR ligands). Based on the finding that most NK cell correlations are in patients with $A M L$, we focused our genetic analysis on patients receiving transplants for AML. We were interested in a model that would incorporate KIR into selection algorithms based on the premise that since most centers do not favor T-cell depleted unrelated donor transplant and acknowledging the importance of HLA-typing in the transplant field which repeatedly shows better outcomes with increasingly high resolution of HLA-match. Since HLA interact with KIR, choosing donors by KIR genotyping is a logical biologic interaction to exploit. In an initial series of over 400 donor recipient pairs, the KIR type of the recipient had no effect. In contrast, donors either heterozygous or homozygous for $B$ KIR haplotypes conferred a $30 \%$ improvement in the relative risk of relapse-free survival compared to donors homozygous for $A$ KIR haplotypes. The protective effect was seen in HLA-matched and HLA-mismatched patients but in this initial cohort it was not clear whether the improved survival was a result of relapse protection or decreased treatment related mortality (TRM), perhaps from infection protection or an interaction with GVHD. Therefore, in an effort to better understand this biology, we derived a new classification of relevant immunogenetic KIR loci. These were based on 27 unique KIR haplotype sequences, allowing division of 4 centromeric or telomeric gene content motifs which define subsets of the KIR $A$ and $B$ haplotypes. KIR $A$ haplotypes contain a Cen-A motif (defined by the presence of the inhibitory KIR gene $2 D L 3$ ) and a Tel-A motif (defined by the presence of the activating gene 2DS4). The $B$ haplotypes are defined by Cen-B (presence of $2 D S 2$ and $2 D L 2$ ) and/or Tel-B (presence of 2DS1 domains). Based on these defined gene content characteristics, we classified donor KIR genotypes as homozygous A/A or defined by the type (Cen-B or Tel-B) or the number of $B$ domains $(0,1,2$, or $>3$ B-content score) in 1,086 AML and $334 \mathrm{ALL}$ patients undergoing URD transplantation. We identified favorable relapsefree survival (RFS) and protection against relapse associated with donor KIR B genotypes vs. the A genotypes. In transplants using the $\sim 11 \%$ who were homozygous for the Cen- $B$ motif, compared to KIR $A$ haplotype donor $\mathrm{HCT}$, using these donors led to significantly improved RFS (RR 0.72: $95 \% \mathrm{Cl}(0.55-0.94), \mathrm{p}=0.014)$. Most strikingly, homozygous Cen- $B / B$ donors conferred significant protection against relapse (RR of relapse $0.34: 95 \% \mathrm{Cl}(0.2-0.57), p=0.0001$ ) with absolute relapse rates of only $15 \%$ in Cen-B/B vs. $31 \%$ in Cen$A / A$ donors. Multivariate modeling demonstrated that donors with higher KIR B-content scores resulted in significantly improved RFS: 2B motifs (RR 0.78: 95\% Cl (0.63-0.95), $\mathrm{p}=0.013$ ) or $\geq 3 \mathrm{~B}$ motifs ( $R R$ 0.76: $95 \% \mathrm{Cl}(0.57-1.02), \mathrm{p}=0.07)$. Higher KIR B-content scores also yielded lesser risks of relapse: $2 \mathrm{~B}$ motifs (RR $0.54: 95 \% \mathrm{Cl}(0.39-0.74)$, $\mathrm{p}=0.0001)$ or $>3 \mathrm{~B}$ motifs (RR $0.45: 95 \% \mathrm{Cl}(0.27-0.74), \mathrm{p}=0.0017)$. These favorable donor KIR genotypes had no impact on the rates of GVHD or TRM. Somewhat surprisingly, the KIR B donors did not yield improvements in outcome for URD transplants in patients with ALL. Therefore, these data suggest that AML blasts may be particularly sensitive to killing NK cells and suggest that activating genes present in the KIR B haplotype may recognize unique ligands on AML blasts. Our conclusion from these studies is that donors can be stratified by KIR into those with Best (Cen-B homozygous present in $11 \%$ of the population), Better ( $>2$ B defining domains as seen in $20 \%$ of the population) or Neutral donor KIR genotypes. A publically available calculator to determine this stratification is available on-line (http://www.ebi.ac.uk/ipd/kir/donor_b_content.html). KIR genotyping as few as 3 of the best HLA matched donor candidates should substantially increase the frequency of URD transplants from donors with favorable KIR gene content (from 31\% to 79\%) 
and a multi-center trial in the United States is underway to test this strategy prospectively. We are also trying to understand if KIR and other receptors may allow us to exploit adoptive transfer of NK cells for therapeutic use in patients with advanced cancers. One main challenge in the field is whether these results can be extrapolated beyond AML to other hematologic malignancies and solid tumors, and several studies are in progress to address these issues. In conclusion, better ways to optimize NK cell effector function, whether through cytokines such as IL-15, or through inflammation induced by infection (as we have shown with human CMV reactivation after transplantation), will enhance innate immune responses for therapeutic benefit.

\section{Novel strategies for Ex Vivo Expansion Human Umbilical Cord Blood (HUCB) \\ Arnon Nagler, Lena Ribakovsky and Avichai Shimoni}

Division of Hematology and CBB, Chaim Sheba Medical Center, Tel Hashomer, Israel

Adult Human Umbilical Cord Blood (HUCB) transplantation (CBT) is steadily increasing in the last few years reaching already few hundred per year. The principal drawbacks of adult CBT are the limited number of hematopoietic stem cells presenting in the HUCB graft resulting in both longer time to engraftment as well as higher nonengraftment rates in comparison to bone marrow (BM) or mobilized peripheral blood stem cell transplantation (MPBSC) transplantation $(1,2)$. This slower myeloid and platelet engraftment post CBT results in higher day 100 mortality as compared to other types of alogeneic transplantation (AlloSCT) (3). several strategies including co-transplantation of more than one HUCB unit (4), expansion of HUCB ex vivo (5), non-myeloablative conditioning (6), simultaneous infusion of HUCB and highly purified haploidentical stem cells (7), co-transplantation of HUCB and mesenchymal cells (8) and intraosseous CBT (9) are currently explored in clinical trials. One of the leading strategies to overcome the delayed short term engraftment post CBT in ex vivo expansion of the HUCB derived stem cell, taking advantage of the higher expansion potential of HUCB derived hematopoietic stem cells in comparison to their counterparts from BM or MPBSC. Preclinical studies have demonstrated the superior proliferative potential of $\mathrm{CB}$ derived HSC. Cytokine stimulated proliferation of $C B$ stem cells was found to be 80 - fold better than that of $B M$ progenitors (10). Moreover, $C B$, but not adult BM stem cells, were shown to repopulate non diabetic severe combined immune deficient (NOD/SCID) mice without aid by exogenous cytokines (11). The first attempt to clinically ex vivo expand HUCB was performed by Kurtzberg group using AastromReplicell system and a cocktail of cytokines that includes PIXY321, flt-3 ligand and erythropoietin (12). Shpall et al expended CD34+ cells isolated from one of two HUCB fractions with SCF, TPO and G-CSF. After 10 days in culture the TNC dose increased 56 -fold and $\mathrm{CD}_{34}{ }^{+}$cells increased four- fold. Thirty seven patients were co-administered with one expanded and one unmanipulated $C B$ fractions. No engraftment hastening was detected (13).

Later on, McNiece et al. applied a two-step, 14-days expansion protocol which yields and increase in TNC as great as 400 -fold and a 20 -fold increase in the $\mathrm{CD}_{3} 4^{+}$cells. 71 patients were randomized for transplantation with two unmanipulated $\mathrm{CB}$ units or one unmanipulated and one expanded CB unit using a two-step strategy (14). Both units were infused on day 0 . The patients who received one expanded and one unmanipulated CB units showed a significantly faster neutrophil recovery median of 7 days (range 4-15 days, $n=14$ ) compared to 14 days (range $5-32$ days, $n=12$ ) for those administered with two unmanipulated CB units. Interestingly, several months after transplantation chimerism analysis showed only the unmanipulated CB unit. Nevertheless, short- term progenitors from the expanded portion may provide faster transient engraftment, reducing early morbidity and mortality, while the unmanipulated $\mathrm{CB}$ would provide sustained hematopoesis.

Another approach to improve the expansion outcome is using novel agents that transiently inhibit HSC differentiation and thus give the HSC proliferation advantage. For example, the copper chelator tetraethylenepentamine (TEPA) and the histone deacetylase inhibitor (HDAC) - valproic acid have been shown to transiently block differentiation of $\mathrm{CB}$ stem cells thus enhancing their proliferation. TEPA and HDAC are now at the stage of phase II clinical trials $(5,15)$.

With the recent advances in understanding the molecular mechanisms involved in proliferation and maintenance of $\mathrm{HSCS}$, additional candidates for further manipulation of HSCs have been introduced. Such are Notch-1, Wnt/B-catenin, the receptor tyrosine kinase Tie2, transcriptional factor Bmi-1 and homebox genes HoxB. Delaney et al (16, update personal communication) reported on 10 patients with high risk leukemia transplanted with a combination, of one unmanipulated and one expanded CB units using engineered Notch delta-1 ligand and cytokines cocktail. Expansion resulted in the average increase in $\mathrm{CD}_{3} 4^{+}$population of 160 -fold and in TNC number of 660 fold. The time to neutrophil recovery was 14.5 days (range 7-34) in expansion arm compared to 26 days (range $16-48, n=17$ ) in recipients of two unmanipulated CB units. Additional attempts to ex vivo expand HUCB is incubation on native or engineered stroma. Shpall et aldemonstrated in vitro the the advantage of ex vivo expansion of HUCB using stroma in comparison to liquid culture (17). Using this strategy the MD Anderson group recently performed a clinical trial in the 32 patients with

Table 1: Strategies for Facilitating Engraftment in Cord Blood Transplantation

\begin{tabular}{|l|}
\hline Co-transplantation of more than one CB unit (4) \\
\hline Expansion of CD34+ progenitor cells (5) \\
\hline Non-myeloablative conditioning (6) \\
\hline Simultaneous infusion of CB and highly purified haploidentical stem cells (7) \\
\hline Co-transplantation of CB and mesanchymal cells (8) \\
\hline Intra-osseous CBT (9) \\
\hline
\end{tabular}

Table 2: Strategies for Ex Vivo Expansion of Cord Blood Hematopoietic Stem Cells

\begin{tabular}{|l|}
\hline Expansion for cytokine cocktail $(13)$ \\
\hline Expansion on microenvironment mimicking device (12) \\
\hline Expansion with differentiation blocking agents $(5,16)$ \\
\hline Copper chelation (5) \\
\hline Notch ligand (16) \\
\hline Expantion on bone marrow stroma (17) \\
\hline Expansion on engineering stroma (Stro-1+, Angioblast) (18) \\
\hline Expansion using MDR based technology $(20)$ \\
\hline
\end{tabular}


various hematological malignancies using the Stro- 1 based engineered third party stroma (18). Other are using placenta derived mesenchimal like cells grown on bioreactor (19). Finally, we are developing another novel approach for ex vivo expansion of HUCB based on the fact that CB derived CD34+ and CD133+ HSC express 5 fold more PgP than more mature cells, thus enable ex vivo expansion with multi drug resistant (MDR) substrate like colchicine (20).

In conclusion, expansion of $C B$ hematopoietic stem cell is feasible and safe. In the last 10 years the technology has moved from the bench to the clinic. Several Phase I and II clinical trials have been or are currently being performed. It seems that cord blood hematopoietic stem cell expansion is able to shorten engraftment and reduce day 100 mortality post CBT. Only future will tell if ex vivo expansion of cord blood will become the leading technology and the common clinical practice aiming in facilitating engraftment and reducing TRM after CBT.

\section{References}

1. Stanevsky A, Shimoni A, Yerushami R, Nagler A. Cord blood stem cell for hematopoietic transplantation. Stem cell Rev 2011; 7:425-433.

2. Stanevsky A, Goldstein G, Nagler A. Umbilical cord blood transplantation: pros, cons and beyond. Blood Rev 2009; 23:199-204.

3. Cohen YC, Scaradavou A, Stevens CE, Rubinstein P, Gluckman E, Rocha V, Horowitz MM, Eapen M, Nagler A, Shpall EJ, Laughlin MJ, Daniely $Y$ Pacheco D, Barishev R, Olmer L, Freedman LS. Factors affecting mortality following myeloablative cord blood transplantation in adults: a pooled analysis of three international registries. Bone Marrow Transplant 2011; 46:70-76.

4. Verneris MR, Brunstein CG, Barker J, MacMillan ML, DeFor T, McKenna DH, Burke MJ, Blazar BR, Miller JS, McGlave PB, Weisdorf DJ, Wagner JE. Relapse risk after umbilical cord blood transplantation: enhanced graft-versus-leukemia effect in recipients of 2 units. Blood 2009; 114: 4293-4299.

5. de Lima M, McMannis J, Gee A, Komanduri K, Couriel D, Andersson BS, Hosing C, Khouri I, Jones R, Champlin R, Karandish S, Sadeghi T, Peled T, Grynspan F, Daniely Y, Nagler A, Shpall EJ. Transplantation of ex vivo expanded cord blood cells using the copper chelator tetraethylenepentamine: a phase I/II clinical trial. Bone Marrow Transplant 2008; 41:771-778.

6. Brunstein CG, Barker JN, Weisdorf DJ, DeFor TE, Miller JS, Blazar BR, McGlave PB, Wagner JE Umbilical cord blood transplantation after nonmyeloablative conditioning: impact on transplantation outcomes in 110 adults with hematologic disease. Blood 2007; 110:3064-3070.

7. Magro E, Regidor C, Cabrera R, Sanjuán I, Forès R, Garcia-Marco JA, Ruiz E, Gil S, Bautista G, Millán I, Madrigal A, Fernandez MN. Early hematopoietic recovery after single unit unrelated cord blood transplantation in adults supported by co-infusion of mobilized stem cells from a third party donor. Haematologica 2006; 91:640-648.

8. Transplantation of mesenchymal stem cells to enhance engraftment of hematopoietic stem cells. Le Blanc K, Samuelsson H, Gustafsson B, Remberger M, Sundberg B, Arvidson J, Ljungman P, Lönnies H, Nava S, Ringdén O. Leukemia 2007; 21:1733-1738.

9. Frassoni F, Gualandi F, Podestà $M$, Raiola AM, Ibatici A, Piaggio G, Sessarego M, Sessarego N, Gobbi M, Sacchi N, Labopin M, Bacigalupo A. Direct intrabone transplant of unrelated cord-blood cells in acute leukaemia: a phase I/II study. Lancet Oncol 2008; 9:831-939.

10. van de Ven C, Ishizawa L, Law P, Cairo MS. IL-11 in combination with SLF and G-CSF or GM-CSF significantly increases expansion of isolated CD34+ cell population from cord blood vs. adult bone marrow. Exp Hematol 1995; 23:1289-1295.

11. Vormoor J, Lapidot T, Pflumio F et al. Immature human cord blood progenitors engraft and proliferate to high levels in severe combined immunodeficient mice. Blood 1994; 83:2489-2497.

12. Jaroscak J, Goltry K, Smith A, Waters-Pick B, Martin PL, Driscoll TA, Howrey R, Chao N, Douville J, Burhop S, Fu P, Kurtzberg J. Augmentation of umbilical cord blood (UCB) transplantation with ex vivo-expanded UCB cells: results of a phase 1 trial using the AastromReplicell System. Blood 2003; 101:5061-5067.

13. Shpall EJ, Quinones R, Giller R et al. Transplantation of ex vivo expanded cord blood. Biol Blood Marrow Transplant 2002; 8:368-376.

14. De Lima M, McMannis JD, Saliba R et al. Double Cord Blood Transplantation (CBT) with and without Ex-Vivo Expansion (EXP): A Randomized, Controlled Study. ASH Annual Meeting Abstracts 2008; 112:154.

15. Bug G, Gul H, Schwarz K et al. Valproic acid stimulates proliferation and self-renewal of hematopoietic stem cells. Cancer Res 2005; 65:2537-41.

16. Delaney C, Heimfeld S, Brashem-Stein C, Voorhies H, Manger RL, Bernstein ID. Notch-mediated expansion of human cord blood progenitor cells capable of rapid myeloid reconstitution. Nat Med 2010; 16:232-6.

17. Robinson SN, Ng J, Niu T, Yang H, McMannis JD, Karandish S, Kaur I, Fu P, Del Angel M, Messinger R, Flagge F, de Lima M, Decker W, Xing D, Champlin R, Shpall EJ. Superior ex vivo cord blood expansion following co-culture with bone marrow-derived mesenchymal stem cells. Bone Marrow Transplant 2006; 37:359-66.

18. McMannis JD, Hosing C, Kebriaei $P$ et al. Rapid engraftment of Neutrophils and platelets with mesenchymal stem cell based cord blood (CB) expansion (EXP). Blood 2009; 114 (Suppl 1): 1318

19. Grynspan F, Marikovsky, Landau E, Peled T, Nagler A, Holbova R, Guetta $V$, Fuchs S, Feinberg S, Leor J. Ev-vivo expanded human bone marrow derived AC133+ cells to treat myocardial infarction. Blood 2004; 104 (Suppl 1):47a

20. Galski H, Bar I, Nagler A. A novel P-glycoprotein (Pgp) dependent ex vivo approach for expansion of human umbilical cord blood CD133+ cells significantly enriches the CD34+/CD38- fraction. BMT 2009; 43 (Suppl 1): S106.

\section{Current role of allogeneic stem cell transplantation in the management of chronic myeloid leukaemia} Eduardo Olavarria

Complejo Hospitalario de Navarra, Pamplona, Spain

\section{Introduction}

Imatinib has been for over 10 years the treatment of choice for all newly diagnosed patients with $C M L .^{1-4}$ Recently, $2^{\text {nd }}$ generation tyrosine kinase inhibitors (TKI) like Dasatinib and Nilotinib have shown superior efficacy compared to Imatinib in achieving complete cytogenetic remissions and major molecular remissions..$^{5-6}$ It is of note, however, that long-term clinical experience with secondgeneration $B C R-A B L$ inhibitors is only available from the secondline treatment of CML-CP. ${ }^{7-8}$

A new concept is emerging: TKI therapy should be exploited and maximise for all patients from diagnosis (with a judicious use of all available TKI) in order to avoid and minimise failure and sub-optimal responses. After TKI therapy failure, the decision to proceed with allogeneic SCT should be based on the risk of the transplant (EBMT risk score) and the likelihood of responding to a different TKI. Clonal evolution, failure to achieve at least mCyR to Imatinib, high Sokal score, loss of a previously achieved CHR, mutations resistant to $2^{\text {nd }}$ generation TKI and progression to accelerated or blast phase are some of the factors to be considered. Prior exposure to Imatinib does not seem to impact negatively on transplant related mortality, although information regarding the pre-SCT use of Dasatinib and Nilotinib is lacking. Reduced intensity regimens have decreased the toxicity of allogeneic SCT and T cell depletion strategies have successfully reduced the risk of GVHD, but there is still a high relapse rate in CML patients. Imatinib and, to a lesser extent, Dasatinib and Nilotinib have been used to successfully postpone the need for $\mathrm{DLI}$ in this context and to treat relapse of CML at different stages after SCT.

\section{Management of patients with resistance to TKI}

The optimal management of patients who are 'failing' or have 'suboptimal' responses to TKI is far from clear. It is self-evident that one of the causes of seeming resistance to Imatinib is failure to take the 
drug. Although there are no data regarding adherence to second generation TKIs, poor compliance is a problem well-known within the pharmaceutical industry particularly with long-term medication for chronic disorders. Assuming that the TKI is being taken the approach to management will vary with the definition of resistance.

Failure to achieve CCyR by $\mathbf{1 2}$ months: If there is a poor cytogenetic response by 12 months with no evidence of a decline then mutation analysis is indicated. The presence of a T315I mutation would be an indication for allogeneic SCT whenever possible. If there is no mutation or the mutant is sensitive to the second generation TKI, then such therapy would be indicated. However, once resistance to $2^{\text {nd }}$ generation TKI has been established, then an allogeneic SCT should be performed in patients with an EBMT score of 0-5.

Failure to achieve major molecular response: The more recent update of the IRIS Study suggests very little difference in survival between patients in CCyR with or without a MMR. ${ }^{4}$ This makes the current management of this group more difficult but it would be reasonable to continue on the same TKI or to change to a different one. No clear indication for allogeneic SCT should be made in this group of patients at present.

Loss of haematological, cytogenetic and molecular response: Loss of CCyR or CHR indicates a higher degree of tumour load. In this situation the nature of the mutation might direct treatment. In the absence of a mutation then a trial with a different TKI would be reasonable, although the trial should be time-limited. Patients with a low EBMT score ${ }^{15}(0-2)$ could proceed to SCT at the time of best response to $2^{\text {nd }}$ generation TKI therapy. Responses to nilotinib and dasatinib are usually obtained early (6-12 months) and in the absence of such a response all patients suitable for allogeneic SCT should be offered this procedure; regardless of their EBMT score.

\section{Progression to Advanced Phase}

Progression to accelerated phase or blast crisis whilst on TKI therapy is indicative of a clinical situation that is incurable without allogeneic SCT. For those patients of a suitable age and with a suitable donor every effort should be made to achieve a second CP (either with conventional AML-like chemotherapy or with new TKI). Once obtained, an allogeneic SCT should be performed as soon as possible, since responses are short-lived. For patients unsuitable for allogeneic SCT then the aim of the treatment is to prolong survival.

\section{Indications for Stem Cell Transplantation}

In principle, all patients under 55 years should be considered potential HCT candidates if they fail to maintain a response to TKI. Patients 55-70 years might also be considered for HCT with reduced intensity conditioning regimens.

The ability to use HCT effectively depends on timely identification of a suitable donor. The search for such a donor should be undertaken as soon as there is an indication that a patient is at risk for requiring second line therapy. A donor search should be initiated immediately for patients with accelerated phase or blast crisis at diagnosis; for patients with chronic phase disease the donor search should start at onset of warnings, suboptimal response or failure.

\section{First line therapy}

For patients in chronic phase, the role of allogeneis SCT is very limited and should be restricted to exceptional cases. Early HSCT might only be considered at the patient's own request in young (< 40 years) patients with high Sokal/Hasford score and with an EBMT score of 0-2 and the presence of a fully matched donor.

\section{Second line therapy}

Second line therapy for patients who do not achieve a CCyR after judicious use of all TKI available (alone or sequentially) should consider allogeneic SCT for candidate patients with a suitable donor and an EBMT score of $0-2$.

Other patients with a higher EBMT score of 3-5 may proceed to allogeneic SCT if they also: a) had high risk Sokal score at diagnosis; b) achieved less than a minor cytogenetic remission to TKI therapy; c) have evidence of clonal evolution or d) have lost a complete hematological remission.

Second line therapy for patients who relapse after an initial response to TKI should be based on the risk of the transplant (EBMT risk score) and the likelihood of responding to a different TKI. Patients with $A B L$ mutations resistant to $2^{\text {nd }}$ generation TKI should proceed to allogeneic SCT directly. Patients without those ABL mutations may proceed to SCT after a second TKI has been initiated. In these cases before a final decision regarding allogeneic SCT is made, careful and early evaluation of their response to a second TKI is warranted. SCT should be considered as preferential therapy, at the time of best response to the second TKI in patients with an EBMT Score 0-2 and one of the following:

- Clonal evolution.

- Failure to achieve at least mayor cytogenetic response to second line TKI therapy in 6-12 months.

SCT should be considered in patients with an EBMT Score 0-5 in the case of failure or intolerance to the second TKI, presentation in accelerated phase at diagnosis and in the case of newly developed $\mathrm{ABL}$ mutations resistant to $2^{\text {nd }}$ generation TKI (ie, T315I mutation). SCT should be considered as preferential therapy, regardless of EBMT score if there is progression to accelerated or blast phase during TKI therapy and after attempts to regain a second chronic phase have been made.

\section{References}

1. Druker BJ, Guilhot F, O'Brien SG et al. Five-year follow-up of patients receiving Imatinib for chronic myeloid leukemia. N Engl J Med 2006; 355:2408-2417.

2. Goldman JM. How I treat chronic myeloid leukemia in the Imatinib era. Blood 2007; 110:2828-2837

3. Baccarani M, Saglio G, Goldman J et al. Evolving concepts in the management of chronic myeloid leukemia: recommendations from an expert panel on behalf of the European Leukemia Net. Blood 2006; 108: 1809-1820.

4. O'Brien SF, Guilhot J, Goldman A et al. International Randomized Study of Interferon Versus STI571 (IRIS) 7-Year Follow-up: Sustained Survival, Low Rate of Transformation and Increased Rate of Major Molecular Response in Patients with Newly Diagnosed Chronic Myeloid Leukemia in Chronic Phase Treated with Imatinib. Blood (ASH Annual Meeting Abstracts) 2008; 112: Abstract 186.

5. Saglio G, Kim DW, Issaragrisil S et al. Nilotinib versus imatinib for newly diagnosed chronic myeloid leukemia. The New England Journal of Medicine 2010; 362(24):2251-9.

6. Kantarjian $\mathrm{H}$, Shah NP, Hochhaus A et al. Dasatinib versus imatinib in newly diagnosed chronic-phase chronic myeloid leukemia. The New England Journal of Medicine 2010; 362(24):2260-70.

7. Shah N et al. ASCO Annual Meeting 2010: Poster Presentation 6512.

8. Kantarjian HM, Giles F, Bhalla K et al. Nilotinib is effective in chronic myeloid leukemia in chronic phase after Imatinib resistance or intolerance: 24-month follow-up results. Blood 2011; 117:1141-1145

9. McSweeney PA, Niederwieser D, Shizuru JA et al. Hematopoietic cell transplantation in older patients with hematologic malignancies: replacing high-dose cytotoxic therapy with graft-versus-tumor effects. Blood 2001; 97:3390-3400.

10. Ringhoffer $M$, Harsdorf S, Schmitt M et al. Reduced-intensity conditioning followed by T-cell depleted allogeneic stem cell transplantation for patients with chronic myeloid leukaemia and minimal residual disease at the time of transplant: high risk of molecular relapse. Br J Haematol 2007; 136:127-130.

11. Dazzi F, Szydlo RM, Craddock C et al. Comparison of single-dose and escalating-dose regimens of donor lymphocyte infusion for relapse after allografting for chronic myeloid leukemia. Blood 2000; 95:67-71.

12. Peggs KS, Thomson K, Hart DP et al. Dose-escalated donor lymphocyte infusions following reduced intensity transplantation: toxicity, chimerism, and disease responses. Blood 2004; 103:1548-1556. 
13. Olavarria $E$, Ottmann OG, Deininger $M$ et al. Response to Imatinib in patients who relapse after allogeneic stem cell transplantation for chronic myeloid leukemia. Leukemia 2003; 17:1707-1712.

14. Olavarria E, Siddique S, Griffiths MJ et al. Post-transplant Imatinib as a strategy to postpone the requirement for immunotherapy in patients undergoing reduced intensity allografts for chronic myeloid leukemia. Blood Dec 2007; 110: 4614-4617

15. Gratwohl A, Hermans J, Goldman JM et al. Risk assessment for patients with chronic myeloid leukaemia before allogeneic blood or marrow transplantation. Chronic Leukemia Working Party of the European Group for Blood and Marrow Transplantation. Lancet 1998; 352:1087-1092.

\section{Unrelated donor transplantation for aplastic anemia Jakob R Passweg}

MS Hematology Division Basel University Hospital, Switzerland

There are 2 first line treatment options available for aplastic anemia bone marrow transplantation from identical sibling donors, used mainly in young patients with donors available, and immunosuppressive treatment (IST) with antithymocyte globulin and cyclosporine. Both treatment options result in prlonged survival in the majority of patients. Typically, patients after transplantation may have long term survival probabilities of $70-80 \%$ with chronic graft versus host disease (GvHD) being a major problem and patients after IST may have response rates of $60-70 \%$ with relapse, nonresponse and secondary clonal complications being resonsible for failure. Overall both treatment options are considered equivalent with transplantation being the preferred option of first line treatment in young patients with severe disease and IST in older patients with nonsevere disease. Alternative donor transplantation using matched unrelated donors were originally thought to be inferior due to higher rates of graft failure and of acute and chronic GvHD resulting typically in long term survival rates of $30-40 \%$ when used in patients without a sibling donor having failed IST. This has changed dramatically in the late 1990 s with long terms survival results approaching those of transplantation using sibling donors. This improvement is most likely due to progress in high resolution typing, thus identifying better matched donors with lower risks of graft failure and of acute and chronic GvHD as compared to historical controls. This led to a reappraisal of the use of unrelated donor transplantation in this disease. There are studies describing outcome of unrelated donor transplantation in aplastic anemia, but there are no controlled trials comparing alternative donor transplantation to other treatment strategies. When to start unrelated donor search: Typically, patients receiving IST as first line treatment will respond within 4-6 months. Unrelated donor searches will usually require about the same time interval to identify a donor. It is therefore reasonable to start a patient without a family donor on IST and decide at the same time whether an unrelated donor transplant is an option in case of nonresponse. If unrelated donor transplantation is an option donor search should start at the time of diagnosis in order to be ready to witch to a transplant strategy if a partial remission has not been achieved after 4-6 months. There are issues to determine up to what upper age limit searches should be started and also whether mismatches are acceptable. Which stem cell source and what type of conditioning for unrelated donor transplantation in aplastic anemia: In sibling donor transplantation the use of marrow has been shown repeatedly to result in lower rates of chronic GvHD than peripheral blood stem cells. Although data in unrelated donor transplantation are less clear it is reasonable to extend the experience made in sibling transplantation to unrelated donor transplantation and use marrow stem cells preferentially. Peripheral blood contains larger numbers of T-lymphocytes explaining the higher chronic GvHD risks. In malignancy this results in higher graft versus leukemia effects but this is not required in a nonmalignant disease such as aplastic anemia. The EBMT has run a study using reduced doses of cyclophsophamide in combination with fludarabine and ATG for conditioning adding $2 \mathrm{~Gy}$ of TBI in adolescents and adults with convincing results. Conclusions: Unrelated donor transplantation has become an accepted and highly effective second line treatment for patients with aplastic anemia.

\section{Transplantation for T-cell lymphoma - Pro auto}

Peter Reimer

Kliniken Essen Süd, Essen, Germany

Peripheral T/NK-cell lymphomas (PTCL) are rare malignancies with a poor prognosis with the exception of ALK+ ALCL (anaplastic lymphoma kinase expressing anaplastic large cell lymphoma). So far, no standard therapy has been defined. This is mainly due to the lack of PTCLrestricted randomised trials and the heterogeneity of most published series. Although CHOP (cyclophosphamide, doxorubicin, vincristine, and prednisone) and CHOP-like regimen are commonly used in the primary therapy of PTCL, this is largely due to the adoption of treatment strategies for aggressive B-cell lymphoma and these protocols have never been established prospectively as a preferred treatment in PTCL. Anthracycline-based polychemotherapy leads to a dismal outcome, with sustaining complete remissions achievable in only a minority of patients. Furthermore, the role of anthracyclines in the first-line treatment of PTCL is a matter of debate since a retrospective international survey did not reveal a significant impact on overall survival (OS) in PTCL.

High-dose therapy with autologous stem cell transplantation (HDTautoSCT) has become the standard of care in relapsing and primary refractory high-grade B-cell lymphomas and seems a sensible approach to overcome chemotherapy resistance in PTCL.

\section{Second-line therapy}

So far, prospective randomized studies on salvage HDT-autoSCT are lacking. To date, at least 16 retrospective studies, each including more than 15 patients have addressed this issue.

These studies were heterogeneous in terms of histological subgroups, patient characteristics, prognostic factors, myeloablative regimen, and duration of follow-up. Taken together, this strategy is feasible and safe with a low morbidity and mortality rate. The OS in these series ranged from $35 \%$ at 2 years to $70 \%$ at 5 years, respectively and the disease-free survival (DFS) or event-free survival (EFS) from $28 \%$ at 2 years to $56 \%$ at 5 years, respectively. Although the earlier reports tend to show somewhat better results than the series published recently, when subgroup or matched control analyses were performed, the OS results for PTCL were equivalent to the long-term outcome in patients with aggressive B-cell lymphomas. The disease status at transplantation seems to correlate with the outcome in most series. In summary, second-line HDT-autoSCT in PTCL is feasible and seems an effective approach for a considerable subgroup of patients.

\section{First-line therapy}

Several retrospective studies on upfront HDT-autoSCT have been published. Like in the salvage setting, a comparison of the cited series is hampered by their variety. The OS in these retrospective studies ranged from $53 \%$ at 3 years to $62-68 \%$ at 5 years. Notably, the DFS/ EFS did not appear to be much lower than the OS in most cohorts indicating a substantial curative potential for this approach in previously untreated PTCL. The EBMT (European Group for Blood and Marrow Transplantation) published the largest study on 146 patients with angioimmunoblastic T-cell lymphoma with an actuarial OS of $67 \%$ at 2 years and $59 \%$ at 4 years. In these retrospective studies chemotherapy-sensitive disease was the major factor predicting OS and PFS. Prospective randomized PTCL-restricted studies are lacking. Two French trials by the GELA (Groupe d'Etude des Lymphomes de l'Adulte) on upfront autoSCT in poor-risk, aggressive NHL also included PTCL (LNH87-2 and LNH93-3 trial). In the intent-to-treat analysis, none of these studies demonstrated a significant benefit for the high-dose arm. However, the limited number of patients in 
the high-dose group and the restriction to high-risk patients, only, do not allow to definitely clarify the impact of first-line HDT-autoSCT in PTCL.

Five larger prospective PTCL-restricted trials have published data on 372 patients with frontline HDT-autoSCT. Compared to the retrospective studies, these prospective series are much more homogeneous. In these trials the OS ranged from 48 to $73 \%$ at 3 years to $34 \%$ at 12 years. The DFS/EFS or the progression-free survival (PFS) were between 36 to $53 \%$ at 3 years and $30 \%$ at 12 years. One challenge of this approach is early progressive disease leading to about one third of patients failing to achieve transplantation. Novel treatment concepts incorporating new agents and/or dose-dense regimen should be further investigated to improve remission status prior to transplantation. With regard to these prospective data, again the remission status at the time of transplantation was a significantly prognostic factor in most. In addition, the IPI and PIT also show prognostic value in some, but not in all series.

In conclusion, HDT-autoSCT offers prolonged remission in about two thirds of the patients with PTCL in the upfront-setting and retrospective series show similar results compared to aggressive B-cell lymphoma as salvage approach. Therefore, taken together the limited data available and in the absence of more effective strategies, upfront HDT-autoSCT seems the most promising and evaluated approach in patients with PTCL, especially when achieving CR after induction therapy.

\section{Prospects for manipulating $\mathrm{T}$ cell responses to minor histocompatibility antigens for promoting leukemia eradication}

Marie Bleakley, Michael Hudecek, Edus H Warren and

Stanley R Riddell

Fred Hutchinson Cancer Research Center, Seattle, WA, USA

Allogeneic hematopoietic stem cell transplantation (HCT) cures many patients with leukemia by providing a graft versus leukemia (GVL) effect mediated in part by donor T cells that recognize HLAbinding peptides termed 'minor histocompatibility (H) antigens' expressed on hematopoietic cells including leukemia. Minor $\mathrm{H}$ antigens are viewed as foreign by the donor immune system and unlike tumor associated self antigens, elicit high avidity $\mathrm{CD} 8^{+}$and $\mathrm{CD} 4^{+}$ $\mathrm{T}$ cell responses that are capable of recognizing multiple determinants expressed both on malignant cells. The potency and diversity of alloreactive $T$ cell responses necessitates the use of immunosuppression post transplant to avoid graft versus host disease (GVHD), and immunosuppression undoubtedly also partially abrogates the GVL effect. Thus, if strategies could be developed to selectively augment $\mathrm{T}$ cell responses to minor $\mathrm{H}$ antigens that are restricted in their expression to recipient hematopoietic cells, this would be predicted to augment the GVL effect without GVHD.

Minor $\mathrm{H}$ antigen-specific T cells have been isolated from $\mathrm{HCT}$ recipients and those that are specific for hematopoietic restricted minor $\mathrm{H}$ antigens have been shown to lyse primary AML and ALL cells in vitro and prevent the engraftment of primary AML in NOD/SCID mice. Minor $\mathrm{H}$ antigen-specific T cells have also been detected by HLA/peptide tetramer ('tetramer') staining of peripheral blood in patients responding to donor lymphocyte infusion (DLI), and the development of minor $\mathrm{H}$ antigen-specific T cells that recognize chronic lymphocytic leukemia has correlated temporally with tumor regression after nonmyeloablative $\mathrm{HCT}$, providing further inferential evidence for their role in leukemia eradication. Direct evidence for leukemia eradication by targeting minor $\mathrm{H}$ antigens comes from adoptive transfer studies by our group in which the infusion of minor $\mathrm{H}$ antigen-specific $T$ cell clones resulted in induction of remission in patients with chemotherapy refractory relapse of acute leukemia after allogeneic HCT. However, on-target toxicity to recipient non-hematopoietic tissues that express the antigen was observed in a subset of patients illustrating the importance of targeting minor $\mathrm{H}$ antigens that are truly expressed selectively on hematopoietic cells to avoid toxicity. Although much has been learned about the mechanisms of minor $\mathrm{H}$ antigen immunogenicity, the expression of specific antigens on normal and malignant cells, and their role in GVL responses, the harsh reality is that too few human minor $\mathrm{H}$ antigens that are expressed selectively on hematopoietic cells have been discovered to permit broad utility in clinical trials of immunotherapy. At the present time only approximately $35 \%$ of recipients of HLA-identical sibling HCT would be candidates for T cell immunotherapy trials to evaluate the safety of targeting one of the known hematopoieticrestricted minor $\mathrm{H}$ antigens. Progress in minor $\mathrm{H}$ antigen discovery has been slow due to technical challenges in isolating $\mathrm{T}$ cell clones, which have been critical reagents for identification of the genes that encode minor $\mathrm{H}$ antigen, and in localizing the underlying polymorphic genetic sequence. Is this a reason to abandon the notion of manipulating $T$ cell alloreactivity to promote tumor eradication? The answer is surely NO. Several advances are rapidly overcoming these obstacles including the development of databases annotating human genetic polymorphism and gene expression profiling of nonhematopoietic tissues and normal and malignant hematopoietic cells that allow the in silico identification of candidate minor $\mathrm{H}$ antigen peptides that exhibit the appropriate tissue distribution and are predicted to bind to HLA alleles. Additionally, high throughput techniques can now rapidly and thoroughly analyze T cell immune responses elicited in vivo or in vitro to define relevant immunogenic targets. These developments are now being employed for minor $\mathrm{H}$ antigen discovery in several labs, and should lay the foundation for novel immunotherapeutic approaches to the problem of leukemia relapse after $\mathrm{HCT}$ that target minor $\mathrm{H}$ antigens on leukemia.

\section{Allogeniec transplantation for standard-risk all in first complete remission \\ Jacob M Rowe}

\section{Rambam Medical Center, Haifa, Israel}

The optimal post remission therapy for acute lymphoblastic leukemia (ALL) is an area that has been controversial for a considerable period of time. Until about a decade or two ago, this was fundamentally divided, within given age groups, into patients who were positive or negative for the Philadelphia chromosome. Immunophenotyping also had an important, but lesser role. More recently, the controversy has ranged around the optimal management of adults who are at standard risk. In this group of patients, the traditional post remission approach is the one based on induction, consolidation and prolonged maintenance therapy. After successful induction and prophylaxis to the central nervous system (CNS), patients are offered consolidation/maintenance therapy for about 2.5-3 years. This approach has been extrapolated from the pediatric experience, although this has never been rigorously tested in the era of modern chemotherapy. Among the pediatric clinical trials, any attempt to shorten this period or reduce the doses has lead to an increased rate of relapse. A more recent approach has been to employ more intensive regimens, both in induction and in the immediate post remission period. Such a regimen has now been adopted in many centers, based on historic comparisons of apparent improved results among adolescents treated in pediatric centers compared with those treated in adult centers. The degree of this intensification is variable among the published reports and it is still uncertain whether the apparent superior results reflect an inherent advantage for these 'pediatric-like' regimens versus the application of a more rigorous and uncompromising culture of protocol drug administration in pediatric centers versus an adult setting. Furthermore, biologic differences may be present in adolescents chosen to be treated in pediatric versus those referred to an adult center. 
Allogeneic bone marrow transplantation represents another approach that has more recently moved into the forefront. This modality provides the most potent anti-leukemia strategy through the immunologically mediated graft-versus-leukemia effect (GVL). Although bone marrow transplantation has been studied for the past two decades, most previous studies were confined to those patients with the Philadelphia chromosome or those with other high risk features. There has only been one large study that investigated bone marrow transplantation for patients at standard risk in a prospective randomized fashion. The International ALL Study, conducted jointly by the Medical Research Council in Great Britain (MRC) and the Eastern Cooperative Oncology Group (ECOG) in the USA, represents a major transatlantic collaboration that was initiated in 1993 and was designed to prospectively define the role of transplantation for all adult patients with ALL. 2000 patients were recruited and the study closed in the year 2006. In this study, patients were prospectively defined as standard risk if they were negative for the Philadelphia chromosome; were less than 35 years of age; had a white cell count less than $30,000 / \mu \mathrm{l}$ for B-lineage or less than $100,000 / \mu \mathrm{l}$ for T-lineage; and if they achieved CR within phase I of therapy (i.e. within 4 weeks). Patients needed to have all these characteristics to be considered at standard risk.

For this very large cohort of patients at standard risk, the complete remission rate was $96 \%$. A genetic randomization was employed, such that patients with a donor were assigned to an allogeneic transplant, to be performed immediately following induction therapy and CNS prophylaxis with high-dose methotrexate. Those without a donor were assigned to standard consolidation/maintenance therapy for 2.5 years. Using an intention-to-treat analysis, the overall survival was $63 \%$ for patients with a donor and $52 \%$ for those without a donor $(p=0.02)$, with a dramatic reduction in relapse rate from $49 \%$ to $24 \%$ for those with a donor. The difference favoring the donor group was present at all age groups, including adolescents in the 15-20 year age group.

It is always possible that innovative approaches, incorporating some of the new monoclonal antibodies or other targeted agents, may improve the overall results for those without a donor. It is also possible that other regimens, using established drugs but in different schedules, or more intensive regimens, may also improve the overall results for those who do not have a donor. However, making comparisons across studies is fraught with hazards. Conflicting clinical data in ALL both for transplant and for non-transplant patients are common. All studies are inherently different; they vary in trial design, in sample size and eligibility criteria. Great caution needs to be applied in extrapolating data from historic cohorts and applying these as standards of care. Suggestions for improvement should be tested against the best arm of an adequately sized randomized study. In this instance, allogeneic transplantation should be the gold standard against which all new therapies should be tested. In 2011, probable exceptions to this, are those with very early MRD negativity (days 11 and 24) and possibly those with the more favorable cytogenetics, such as high hyperdiploidy or del (9q). For the $10 \%$ of patients with very early MRD negativity a non-transplant approach clearly seems appropriate. For other patients, novel approaches should be carefully evaluated in prospective and randomized studies.

\section{The role of the conditioning regimen in preventing relapse after allogeneic stem-cell transplantation in patients with acute myeloid leukemia (AML) and myelodysplastic syndrome (MDS) \\ Avichai Shimoni and Arnon Nagler}

Division of Hematology and Bone Marrow Transplantation, Chaim Sheba Medical Center, Tel-Hashomer, Israel

Allogeneic stem-cell transplantation (SCT) is a curative therapy for patients with AML and MDS. Substantial improvement has been made over the last decade in supportive care methods, such as prevention and treatment of GVHD, infections, and organ damage, resulting in marked reduction of non-relapse mortality (NRM) rate. However, relapse remains the major cause of treatment failure and despite extensive research, relapse rates have not changed significantly. The status of disease at SCT is the most significant factor predicting outcome after SCT. Relapse post-transplant ranges from $25 \%$ in early stage disease to more than $60 \%$ in advanced disease. ${ }^{2}$ Achieving low leukemia burden before SCT, and in particular negative minimal residual disease (MRD) status ${ }^{3}$ as well as early SCT can reduce relapse rate and improve outcome.

The role of dose intensity of the conditioning regimen in patients with AML has been extensively reviewed. ${ }^{4}$ Historically, major efforts were aimed at increasing regimen intensity by increasing the doses or adding additional agents. Clift et al reported a randomized study comparing two doses of Total-body irradiation (TBI) in patients with AML in CR1. Relapse rates were lower after the larger dose, supporting the role of dose intensity in eradicating AML, however, NRM was significantly increased, mostly due to increased rates of GVHD, resulting in similar survival. ${ }^{5}$ Several other studies supported the same outcome. Intensified myeloablative regimens are associated with reduction of relapse but NRM becomes prohibitive. The current standard myeloablative conditioning (MAC) regimens, $\mathrm{Cy} / \mathrm{TBI}$ and BuCy probably represent the maximally tolerated regimens. There is no evidence that outcome after one of these regimens is superior to the other in any setting. These regimens are still the standard regimens for eligible patients. ${ }^{6}$ Further dose intensification or addition of other agents is unlikely to improve patient outcome. Adding non-toxic targeted therapies, such as monoclonal antibodies may show promise, however so far they have shown limited success and none was proven in well designed studies.

Over the last decade the pendulum turned from intensified chemoradiotherapy to induction of graft-versus-leukemia (GvL) effect as the primary goal of therapy. A plethora of new regimens have been designed to reduce NRM and allow treatment of elderly and medically infirm patients. ${ }^{7}$ These regimens vary in myelosuppressive and immune-suppressive intensity from truly nonmyeloablative (NMA) to reduced-intensity regimens (RIC) and up to reduced toxicity myeloablative regimens (RTC). The experience so far with these regimens shows that they allow long-term disease control. ${ }^{8}$ NRM is reduced allowing therapy of patients who are MAC ineligible. Several studies compared outcomes of NMA/RIC and MAC.8-11 None was randomized and all suffered from significant selection bias. However, with this limitation most studies showed reduced NRM, increased relapse and similar survival with RIC compared to MAC. We have shown that survival was similar for all regimens in patients transplanted in early stage disease. However, in patients with advanced disease outcome with RIC was inferior compared to $M A C /$ RTC due to very high relapse rates with RIC in this setting. Similarly, Luger et al. showed in a very large CIBMTR study that outcome after RIC and MAC was similar, but NMA was associated with inferior results due to excess relapse rates. ${ }^{11}$ It seems that the conditioning regimen is needed to achieve an MRD state post-transplant to support a GVL effect and allow time for the generation of GVL. For patients transplanted in remission, especially in MRD negative remission, increasing dose-intensity above the RIC level is not required and is not associated with better outcome. However, NMA intensity may be inferior in achieving this goal. It is unlikely that further reduction of relapse rate in these patients can be achieved by changing the drug combination of the regimen without concomitant increase in NRM. For patients transplanted not in remission, or with highly aggressive biological characteristics predicting rapid progression, an intensive regimen is required to allow cyto-reduction in a sufficient level and duration until GVL is operative. In this setting, intensive regimens with tolerable toxicity are of marked benefit.

Relapse is causes by leukemia cells that survive the chemotherapy regimen and evade the immune response. Quiescent/non-cycling leukemia stem cells (LSC) may be resistant to therapy, may persist 
after transplant and contribute to relapse. Genomic and epigenetic changes may result in therapy resistance. LSC may evade or suppress the immune response. Changes in the chemotherapy regimen are unlikely to overcome these resistance mechanisms. Targeted and immune therapies are more promising in this goal. These therapies can be given pre-transplant to strengthen the depth of remission. They may be combined with the conditioning regimens, and as emphasized recently, may be given at the post transplant period. These therapies can be given prophylacticlly to all patients, or pre-emptively to patients with evidence for MRD post-transplant. These therapies should be non-toxic, non-myelosuppressive, with no interaction with medications given routinely after transplant. Ideally they should enhance GvL without increasing GvHD. 5-Azacytidine has been explored as maintenance therapy after SCT for patients with AML and MDS with promising results. It fulfils most of the criteria for post-transplant therapy. Toxicity and myelosuppression is tolerable and there is evidence that GVL may be enhanced. ${ }^{12}$ Immune therapies may include among others, prophylactic or preemptive donor lymphocyte infusion (DLI), natural-killer cell infusion, vaccines, etc.

In conclusion, BuCy and $\mathrm{Cy} / \mathrm{TBI}$ are still the standard regimens for eligible patients. Relapse rates can be reduced by increasing dose intensity, but this is most often associated with increased NRM, and no change in survival. MAC and RIC probably result in similar outcome at least in patients in remission at the time of SCT. In patients not in remission or with rapidly progressing leukemia, intensified regimens have an advantage. NMA/RIC are associated with highrelapse rates and poor outcome in this setting. Pre- and post-transplant interventions may be more promising than modifications in the conditioning regimen. Pre-transplant interventions are aimed at achieving an MRD negative status at transplantation. Post-transplantation interventions are aimed at eliminating MRD, targeting resistant leukemic populations (LSC), enhancing and sustaining an immune GVL effect. Randomized studies are needed to determine the different merits of these regimens.

\section{References}

1. Gooley TA, Chien JW, Pergam SA et al. Reduced mortality after allogeneic hematopoietic-cell transplantation. N Engl J Med 2010; 363: 2091-2101.

2. Center for International Blood and Marrow Transplantation. 2010 Summary slide set. http://www.cibmtr.org/ReferenceCenter/SlidesReports/ SummarySlides/pages/index.aspx. Accessed November 2, 2011.

3. Walter RB, Gooley TA, Wood BL et al. Impact of pretransplantation minimal residual disease, as detected by multiparametric flow cytometry, on outcome of myeloablative hematopoietic cell transplantation for acute myeloid leukemia. J Clin Oncol 2011; 29: 1190-1197.

4. Shimoni A, Nagler A. Optimizing the conditioning regimen for allogeneic stem-cell transplantation in acute myeloid leukemia; dose intensity is still in need. Best Pract Res Clin Haematol 2011; 24: 369-379.

5. Clift RA, Buckner CD, Appelbaum FR et al. Allogeneic marrow transplantation in patients with acute myeloid leukemia in first remission: a randomized trial of two irradiation regimens. Blood 1990; 76: 1867-1871.

6. Nagler A, Labopin M, Shimoni A et al. Comparison of outcomes after allogeneic SCT for adult patients with AML in remission using either I.V. busulfan plus cyclophosphamide or TBI plus cy in the myeloablative conditioning regimen: an- ALWP-EBMT survey [abstract]. Bone Marrow Transplant 2010; 45(Suppl 2), s11. Abstract 120

7. Champlin R, Khouri I, Shimoni A et al. Harnessing graft-versus-malignancy: non-myeloablative preparative regimens for allogeneic haematopoietic transplantation, an evolving strategy for adoptive immunotherapy. $\mathrm{Br}\rfloor$ Haematol 2000; 111: 18-29.

8. Shimoni A, Hardan I, Shem-Tov N, Yerushalmi R, Nagler A. Allogeneic hematopoietic stem-cell transplantation in AML and MDS using myeloablative versus reduced-intensity conditioning: long-term follow-up. Leukemia 2010; 24: 1050-1052.

9. Shimoni A, Hardan I, Shem-Tov $\mathrm{N}$ et al. Allogeneic hematopoietic stem-cell transplantation in AML and MDS using myeloablative versus reduced-intensity conditioning: the role of dose intensity. Leukemia 2006; 20: 322-28.

10. Aoudjhane M, Labopin M, Gorin NC et al. Comparative outcome of reduced intensity and myeloablative conditioning regimen in HLA identical sibling allogeneic haematopoietic stem cell transplantation for patients older than 50 years of age with acute myeloblastic leukaemia: a retrospective survey from the Acute Leukemia Working Party (ALWP) of the European group for Blood and Marrow Transplantation (EBMT). Leukemia 2005; 19: 2304-2312.

11. Luger SM, Ringdén O, Zhang MJ et al. Similar outcomes using myeloablative vs reduced-intensity allogeneic transplant preparative regimens for AML or MDS. Bone Marrow Transplant 2011; e-pub ahead of print 28 March 2011; doi:10.1038/bmt.2011.69.

12. de Lima M, Giralt S, Thall PF et al. Maintenance therapy with low-dose azacitidine after allogeneic hematopoietic stem cell transplantation for recurrent acute myelogenous leukemia or myelodysplastic syndrome: a dose and schedule finding study. Cancer 2010; 116: 5420-5431.

\section{Recommendation for autologus hematopoietic stem cell (HSC) harvest from peripheral blood (PB) and cryopreservation for the high risk workers around nuclear reactors in Fukushima}

Akihiro Shimosaka

Senior Research Adviser, Institute of Medical Science, University of Tokyo, Japan

\section{Background}

Nuclear fuel melt down occurred in three nuclear reactors and the cooling pool for nuclear fuel for one other reactor was damaged by loss of electricity caused by earthquake and Tsunami on March 11, 2011 in Fukushima, North East Japan. Loss of electricity results in loss of cooling water and caused melt down in reactor and damages on nuclear fuel in storage. Melt down and high temperature generated hydrogen and hydrogen explosion happened two times which caused serious damages on reactor buildings. All four reactors are in just about stable condition and huge amount of nuclear fuel are in the reactor as well as in storage pool which must be taken away and transferred to storage site safely. Capacity of reactors, No.1 is 460 MW, No. 2-No. 4 are 784 MW respectively. Huge reactors with huge amount of nuclear fuel. Before take out nuclear fuel, debris caused by explosion must be taken away. Radiation level is at least over $10 \mathrm{~Sv} / \mathrm{hour}$ because monitor can only measure up to $10 \mathrm{~Sv} /$ hour. Debris may play a role as shield from radiation. If you remove debris around reactor, then much higher radiation level will be expected directly from reactor and may be from leak out fuel. Melted fuel is difficult to handle and take time to remove from reactors. Remaining nuclear fuel in pool are 3108 fuel cells and a lot more in reactor. Total amount is at least 30 times more than atomic bomb used in Hiroshima. Issues is not a low level irradiation circumstance but people have to work in very high level radiation area where final work has to be completed. Estimated time to close nuclear reactor is over 30 years. It may become global hazard if Japan fail to shut down reactors completely.

\section{Why Auto Peripheral Blood Stem Cell (Auto PBSC)}

Past experience indicated, at first irradiated person have to be saved from damage on hematopoiesis. Only possible way is hematopoietic stem cell transplantation when radiation is over certain level. Data from over 800 irradiated persons in 60 irradiation accidents, none of them were saved by allogeneic HSCT. Though hematopoiesis was recovered but GVHD/damage caused by radiation overlap on skin, gut/intestine or lung and could not save from other damages. When you have highly irradiated patient, it is difficult to estimate the level of radiation and have to make decision whether additional conditioning for allo HSCT is needed or not. If you have autologus HSC, you need not estimate radiation level but just administer 
stored HSC and need not have any GVHD prophylaxis. Even highest caution will be taken, still there is a risk of unexpected high level irradiation occurs in such high level radiation area. Auto HSCT may not help every radiated patient but at least can save from certain level radiation exposure. Also today we may be able to use MSC for other damages on skin, gut, intestine or lung. Then chances for saving patient from high irradiation will be more. We do not want to lose patient only because of hematopoiesis and would like to try next step to save highly irradiated patient.

This is very similar to insurance coverage for those who are traveling to high risk infectious disease area. For Japan case, companies who are marketing G-CSF and apheresis machine donated G-CSF and apheresis kits for autologus peripheral blood harvest and other company offered storing harvested HSC free of charge voluntary. Even other company offered use of stem cell mobilization accelerating agent for acute use. Thus those who are expected to work in such circumstances, auto peripheral blood stem cell harvest and storage will be good insurance though it may not be able to save $100 \%$.

There is minimum risk for harvesting auto PBSC and in case, irradiated, auto PBSC is the best source for hematopoietic recovery.

I wonder is there any other idea when you have patient exposed to high level radiation and loosing hematopoiesis ability. Level of irradiation is not known. Auto recovery is only known after recovery. I am not confident when patient come in with high level radiation, to judge that there is a chance for auto recovery. I am very comfortable if there is stored autologus stem cell ready for use.

\section{Controversies in stem cell transplantation; in favor of second allogeneic stem cell transplantation for treatment of relapsed hematological malignancies \\ Shimon Slavin}

Professor of Medicine, Scientific \& Medical Director, The International Center for Cell Therapy \& Cancer Immunotherapy (CTCI), Weizman Center, 14 Weizman Street, Tel Aviv 64239,

ISRAEL, Tel: +972-77 777 9255, Fax: +972-77 7779247.

E-mail: slavin@CTCIcenter.com

\section{Introduction and scientific background}

Allogeneic stem cell transplantation (SCT) is considered the penultimate treatment of choice for hematological malignancies whenever malignant cells cannot be eliminated by conventional chemotherapy and/or other available anti-cancer agents, or whenever patients are at a major risk of recurrent disease despite optimal conventional treatment. In general there are two different principles behind the use of allogeneic SCT, which can explain the improved efficacy of allogeneic SCT over conventional chemotherapy:

1. The feasibility to use higher than conventional doses of chemoradiotherapy at myeloablative doses followed by rescue of donor stem cells.

2. Replacement of patient's susceptible non-tumor reactive immune system with donor hematopoietic system including alloreactive lymphocytes which can eliminate residual malignant cells resistant to chemoradiotherapy by an immune mechanism resulting in "reverse rejection" through induction of the so-called graft vs. malignancy (GVM) or graft-versusleukemia (GVL) effects. Whereas at the early era of allogeneic SCT much attention was given to maximize the myeloablative conditioning in order to try to eliminate all malignant cells prior to stem cell transplantation, using stem cells as a rescue procedure, the trend in recent years has become to use the transplant procedure as a means for induction of host vs. graft unresponsiveness, thus allowing durable engraftment of donors' immunohematopoietic system, focusing on elimination of residual malignant cells post grafting by alloreactive donor lymphocytes.
Based on the latter working hypothesis, the trend over the years was to reduce the intensity of the conditioning, which was responsible for procedure-related toxicity and mortality. The discovery of the feasibility to eliminate all malignant cells in patients relapsing following myeloablative SCT by donor lymphocyte infusion (DLI) 25 years ago by Slavin and colleagues (1-3), supported by other investigators $(4,5)$, thus providing a second chance for cure for patients relapsing following maximally tolerated myeloablative conditioning opened the era for development of new protocols that focused on cell-mediated immunotherapy rather than merely maximizing the dose of chemoradiotherapy. Successful post grafting induction of immune mediated GVL or GVM effects by alloreactive donor lymphocytes gave us of the idea to reduce the intensity of the toxic conditioning and focus on post transplant cell-mediated immunotherapy of residual malignant cells. This working hypothesis resulted in development of a variety of reduced intensity conditioning (RIC) and even non-myeloablative stem cell transplantation (NST). Using RIC and NST, transplant related complications were markedly reduced, thus allowing safer SCT for children and young adults while avoiding growth retardation, multiple endocrine deficiencies, cataract formation and sterility. At the other end of the spectrum, these procedures provided a chance for cure for otherwise incurable elderly individuals and for patients in less than optimal performance status who previously could not be considered eligible for conventional myeloablative SCT. The discovery of the feasibility to eliminate malignant cells in patients relapsing following transplantation by DLI 25 years ago (3) provided a second chance for cure for patients relapsing following maximally tolerated myeloablative chemoradiotherapy but unfortunately, in many cases GVL and GVM effects induced by DLI also resulted in severe acute and chronic graft vs. host disease (GVHD) and in some cases, tumor progression persisted despite development of severe GVHD. Obviously, when GVHD occurred, the need for post-transplant immunosuppressive treatment to control GVHD also limited the immune mediated GVL and GVM effects, thus limiting the therapeutic potential of DLI.

\section{New concepts for haploidentical stem cell transplantation for treatment of chemotherapy resistant hematological malignancies focusing on post transplant cell-mediated immunotherapy by donor NK cells}

Based on the above, there seems to be a real need to develop new protocols for treatment of patients failing allogeneic SCT +/$\mathrm{DLI}$ and high risk patients not anticipated to benefit from even a successful myeloablative SCT, while in parallel avoiding or minimizing the risk of acute ad chronic GVHD. Although it has been debatable whether patients relapsing after allogeneic SCT could still be rescued with another procedure, our prior clinical experience and data suggest that cure might be accomplished even in patients who failed myeloablative SCT and DLI and even if such patients entered the transplant procedure in full relapse. The solution seems to be a second or even third allogeneic SCT based on the use of RIC using SCT from haploidentically mismatched donor, followed by post-transplant immunotherapy with activated natural killer (NK) cells $(6,7)$. The rational and reason why such a procedure based on the use of haploidentical stem cell transplantation on the one hand, and post-transplant immunotherapy with donor derived NK cells on the other, can be summarized as follows:

1. Haploidentically mismatched donor is available for every patient in need using parent to child or child to parent or any half-matched family member so there is no need to waste time searching for matched unrelated donor (MUD) which is time consuming, not always successful, and frequently complicated by severe GVHD.

2. Due to the immune mediated GVL and GVM effects, the more mismatched the stronger GVM effects anticipated and therefore a priory, haploidentical lymphocytes focusing on maximally activated NK cells are likely to induce more potent GVL and GVM 
effects then lymphocytes obtained from matched sibling or matched unrelated donor.

3. Whereas in transplants done with fully matched siblings or MUD, GVL effects is mediated predominantly by alloreactive T cells, development of GVHD is the rule in the large majority of patients despite optimal HLA matching and this cannot be prevented even by using optimal post-transplant immunosuppression. Furthermore, the mandatory post grafting immunosuppressive treatment indicated to prevent or treat GVHD negates the therapeutic benefits of GVL and GVM effects. In contrast, following haploidentical SCT, T cells depletion ex vivo prior to transplantation or in vivo post transplantation is mandatory and therefore GVHD is fully preventable in the large majority of cases, and consequently, no post grafting immunosuppressive treatment is required.

4. Following haploidentical SCT optimal GVL effects can be mediated by donor derived IL-2 activated NK cells using either depletion of CD3-positive lymphocytes or positive selection of CD56-positive lymphocytes with immunomagnetic beads. Consequently, since exclusively T cells induce GVHD, the use of NK cells for induction of GVM effects does not cause GVHD.

Taking together, the feasibility to accomplish a relatively safe successful transplantation from related haploidentically mismatched donor provides an opportunity to induce much more potent GVL and GVM effect while avoiding GVHD. This explains why haploidentical SCT can rescue patients not in complete remission after failing prior standard allogeneic SCT from a matched sic or MUD.

Following the aforementioned considerations, we have pioneered the use of haploidentical SCT starting more than 10 years ago for patients relapsing following SCT from a matched sibling or MUD. Indeed, we have already confirmed that all of the four mentioned theoretical considerations are applicable at the patient's bed side and consequently, we have managed to cure patients with aggressive leukemia relapsing following prior SCT with a matched sibling, MUD and even one patient that failed two myeloablative SCT procedures, now $>10$ years out with no evidence of disease, never experiencing any sign of acute or chronic GVHD. Interestingly, patients with acute leukemia can be cured even when transplanted in full chemotherapy-resistant relapse, thus confirming the unique efficacy of GVL effects induced with mismatched immune lymphocytes.

\section{Conclusions}

Considering our successful clinical experience that supports our working hypothesis, we are strong believers that cure may be accomplished in a significant number of patients using haploidentical SCT after failure of prior allogeneic SCT. Cure of leukemia in this setting may be accomplished even if patients are treated in full chemotherapy resistant relapse. Based on the above, it may be advisable to treat patients relapsing following allogeneic SCT with haploidentical stem cell transplantation as long as their performance status is good, rather than trying to induce remission with exhausting doses of hazardous chemotherapy which may not be accomplished or at best may result in higher transplant related risks following SCT from a haploidentical donor. Larger number of patients should be investigated in parallel with improving the conditioning regimen as greater experience accumulates, focusing on minimizing transplant-related toxicity while in parallel amplifying tumor selective immune-mediated GVL and GVM effects.

\section{References}

1. Slavin S, Or R, Naparstek E, Ackerstein A, Weiss L. Cellular - mediated immunotherapy of leukemia in conjunction with autologous and allogeneic bone marrow transplantation in experimental animals and man. Blood 72 (Suppl 1):407a, 1988

2. Slavin S, Naparstek E, Nagler A, Ackerstein A, Kapelushnik Y, Or R: Allogeneic cell therapy for relapsed leukemia following bone marrow trans- plantation with donor peripheral blood lymphocytes. Exp Hematol 1995; 23:1553-1562.

3. Slavin S, Naparstek E, Nagler A, Ackerstein A, Samuel S, Kapelushnik J, Brautbar C, Or R. Allogeneic cell therapy with donor peripheral blood cells and recombinant human interleukin-2 to treat leukemia relapse post allogeneic bone marrow transplantation. Blood 1996; 87: 2195-2204.

4. Kolb HJ, Schattenberg A, Goldman JM et al. Graft-versusleukemia effect of donor lymphocyte transfusions in marrow grafted patients. Blood 1995; 86: 2041-50.

5. Collins RH, Shpilber O, Drobyski WR et al. Donor leukocyte infusions in 140 patients with relapsed malignancy after allogeneic bone marrow transplantation. J Clin Oncol 1997; 15:433-44.

6. Slavin S, Shapira MY, Morecki S, Samuel S, Ackerstein A, Gelfand Y, Resnick I, Bitan M, Or R. Immunotherapy for resistant hematologic malignancies using matched or mismatched rlL-2 activated donor lymphocytes positively selected for CD56+ after allogeneic stem cell transplantation for allogeneic cell therapy without GVHD. 45th ASH Annual Meeting. San Diego, California, USA. December 6th-9th, 2003. Blood 2003; 102(11):400b. Abs\#5329.

7. Slavin S, Shapira M, Morecki S et al. Use of matched or mismatched rIL-2 activated donor lymphocytes positively selected for CD56+ for immunotherapy of resistant leukemia after allogeneic stem cell transplantation. American Society of Clinical Oncology (ASCO). New Orleans, Louisana, USA. June 3rd-8th, 2004. American Society of Clinical Oncology 2004: 23;560. Abs\#6516.

\section{Is photopheresis the treatment of choice for chronic GVHD? No \\ Gerard Socié}

Hematology Transplantation, Hospital Saint Louis, Paris, France, E-mail: gerard.socie@sls.aphp.fr

Chronic graft-versus-host disease (cGVHD) remains the leading cause for late morbidity and mortality after allogeneic hematopoietic stem cell transplantation. While half of the patients respond to first-line treatment, steroid refractory cGVHD remains to be associated with significant morbidity and mortality. The latter is defined as failure to respond to standard treatment consisting of $1 \mathrm{mg} / \mathrm{kg} /$ day prednisone after 8-12 weeks of treatment or progression of CGVHD after 4 weeks of treatment. While primary treatment of CGVHD is based on controlled trials and consists of prednisone given with or without a calcineurin inhibitor, evidence in steroid refractory cGVHD is limited almost exclusively to phase II trials or retrospective analyses. Until recently, no valid criteria for the diagnosis and staging of cGVHD severity were available, which limits the value of most reported trials on treatment of cGVHD. Though not yet validated in a prospective fashion, the NIH consensus criteria on diagnosis and staging of CGVHD as well as on treatment response criteria, reported in 2005, now provide defined criteria which should improve the validity of future results on treatment of cGVHD in the future. At present, most of the available evidence relates to retrospective analyses and studies which did not use uniform criteria for response and did not provide details on severity of cGVHD. An additional problem is the heterogeneity of the patients included in the analyses as for some treatment options results in children differ substantially from results achieved in adults. During the last years a substantial number of patients have been treated with extracorporeal photopheresis (ECP) for steroid-dependent or steroid-refractory cGVHD. The mechanisms of action are complex and not fully understood. ECP reportedly induces apoptosis in all leukocyte subsets, inhibits pro-inflammatory cytokine production, increases production of anti-inflammatory cytokines, reduces stimulation of effector $T$ cells and induces donor-derived regulatory $T$ cells (Tregs). Most of the clinical experience in ECP treatment for patients with CGVHD is based on retrospective analyses including 
limited number of patients with consistently high complete responses of patients with steroid-refractory skin CGVHD responses in other target organs are far from be clearly defined.

Treatment modalities are the use of steroids and calcineurin inhibitors as well as immunomodulating modalities (photopheresis, mTOR-inhibitors, thalidomide, hydroxychloroquine, vitamine A analogues, clofazimine), and cytostatic agents (MMF, MTX, cyclophosphamide, pentostatine). Recent reports showed some efficacy of rituximab, Alemtuzumab and Etanercept in selected patients. Moreover, tyrosin kinase inihibitors such as Imatinib came into the field due to their ability to interfere with the PDGF-R pathway involved in fibrosis. Other treatment options are low dose thoracoabdominal irradiation.

Although different treatment options are available the "trial and error system" remains the only way to identify the drug effective in the individual patient and valid biomarkers are eagerly needed to identify the likelihood of response to a drug in advance. Moreover, the sparse evidence for most treatment entities indicates the urgent need for systematic evaluation of treatment options in second-line treatment options in chronic GVHD.

\section{Maintenance therapy post transplantation in multiple myeloma \\ P Sonneveld}

Erasmus MC, Rotterdam, The Netherlands,

E-mail: p.sonneveld@erasmusmc.nl

High-dose melphalan (HDM followed by autologous stem cell transplantation (ASCT) is considered a standard intensification treatment in multiple myeloma (MM). The aim of post-intensification therapy is either to improve the response by a (short) consolidation or to prolong progression-free survival (PFS) and overall survival (OS), which is known as maintenance. Generally, maintenance treatment is administered for a prolonged time period in order to prevent progression of residual disease.

In this presentation, the role of maintenance after ASCT will be discussed.

Thalidomide is the agent that has been studied most extensively. A recent update to the thalidomide maintenance trials conducted by the IFM and the Arkansas group revealed that after long-term follow up the OS benefit observed for the thalidomide maintenance in the IFM trial disappeared, while in the Arkansas study of TT2 a survival advantage for the thalidomide-arm has become apparent [1-2] Nevertheless, the utility of thalidomide as maintenance therapy is limited due to its neurotoxicity and the shorter survival at relapse that has been observed in the HOVON 50 and the MRC IX studies [3-4].

Two phase 3 trials in CALGB and IFM are investigating lenalidomide as a maintenance treatment post-ASCT [5-6]. Patients were randomized post-ASCT to either lenalidomide maintenance treatment or placebo. Both studies demonstrated a significant improvement in time to progression (TTP) or PFS for the lenalidomide-containing maintenance arm. In both trials the median PFS or TTP was not reached for the lenalidomide maintenance arm, and it was 24 months for the placebo arm in the IFM study and 25.5 months for the placebo arm in the CALGB study. The improvement in PFS was observed in all ISS stages, in all levels of response and independent from del 13 in the French trial. In the CALGB trial, exposure to thalidomide or lenalidomide during induction was not found to influence TTP. In both trials, maintenance treatment with lenalidomide was well tolerated. Although OS data is not yet available, these data are likely to have an impact on treatment practices because of the significant reduction in the risk of relapse associated with lenalidomide maintenance. Lately, an increased incidence of second primary tumors associated with lenalidomide was reported in these trials.
Bortezomib maintenance treatment has been investigated in the HOVON 65 MM / GMMG-HD4 study [7]. Unpublished results show a significant benefit of PFS and OS with bortezomib.

The positive results with novel agents in the post-ASCT setting will require confirmation and refinement on the optimal dose, schedule and duration of treatment, as well as the impact on survival after relapse is needed. In addition, clarification is needed which patients should receive post-ASCT therapy depending on their risk group and prognosis. Finally, longer follow up is needed to assess the impact of post-ASCT on OS.

\section{References}

1. Attal, $M$ et al. Maintenance therapy with thalidomide improves survival in patients with multiple myeloma. Blood 2006; 108(10):3289-94.

2. Barlogie, B et al. Long-term follow-up of autotransplantation trials for multiple myeloma: update of protocols conducted by the intergroupe francophone du myelome, southwest oncology group, and university of arkansas for medical sciences. J Clin Oncol 2010; 28(7):1209-14.

3. Lokhorst, H.M et al. A randomized phase 3 study on the effect of thalidomide combined with adriamycin, dexamethasone, and high-dose melphalan, followed by thalidomide maintenance in patients with multiple myeloma. Blood 2010; 115(6):1113-20.

4. Morgan, $\mathrm{G}$ et al. Maintenance Thalidomide may improve progression free but not overall survival; Results from the Myeloma IX Maintenance randomisation. Blood 2008; 112:245.

5. Attal, $M$ et al. Maintenance treatment with Lenalidomide after transplantation for Myeloma: Final analysis of the IFM 2005-02. Blood 2010; 116(21):141.

6. McCarthy, P.L., Jr., K. Owzar, and K. Anderson. Phase III intergroup trial of lenalidomide versus placebo maintenance therapy following single autologous stem cell transplant (ASCT) for multiple myeloma (MM): CALGB 100104. J Clin Oncol 2010; 28:577s, abstract.

7. Sonneveld, $\mathrm{P}$ et al. HOVON-65/GMMG-HD4 randomized phase III trial comparing bortezomib, adriamycin, dexamethasone (PAD) vs VAD followed by high-dose melphalan (HDM) and maintenance with bortezomib (B) or thalidomide $(\mathrm{T})$ in patients with newly diagnosed multiple myeloma (MM). Blood 2010; 116(21):23.

\section{Prophylactic hematopoietic cell harvesting in nuclear plant workers: the argument against \\ David M Weinstock}

Dana-Farber Cancer Institute, 450 Brookline Avenue, Dana 510B, Boston, MA 02215. Phone: 617 632-4245;

Fax: 617 632-5167.E-mail: dweinstock@partners.org

The March 11, 2011 earthquake and tsunami in Japan resulted in the uncontrolled release of radioactivity from the Fukushima Daiichi nuclear power plant. In the ensuing weeks, dozens of workers received low doses of radiation. Fortunately, no serious radiation injuries have been reported. Occupational limits for radiation exposure among clean-up workers are far below the doses that cause acute organ toxicity. However, it remains possible that accidents or complications could occur during the clean-up and result in dangerous exposures. Based on previous industrial accidents, whole-body exposures over 4 Gray (Gy) can cause severe myelosuppression. Some medical centers in Japan and Europe have offered to preemptively collect hematopoietic progenitor cells (HPC) from workers at Fukushima Daiichi.' If a worker donates autologous HPCs and is subsequently exposed to a significant dose of radiation, the banked cells could be autografted to "rescue" bone marrow function.

Autologous HPC transplantation has been used therapeutically for over 30 years. The procedure makes it possible to treat patients with myeloablative doses of radiation and/or chemo- 
therapy. However, treatment doses are still limited by toxicity to other organ systems (e.g. pulmonary, gastrointestinal, neurovascular). Thus, the benefit from autologous HPC transplantation is limited to doses that do not cause nonhematopoietic lethality. Of paramount importance is dose-rate, which is typically low (0.04-0.5 Gy/min) in therapeutic radiation but can be very high (>1-10 Gy/min) in accidental exposures. Higher dose-rates cause markedly greater toxicity for the same dose, so comparisons of dose-response between industrial accidents and therapeutic irradiation may be grossly inaccurate. ${ }^{2}$

So, what is the likelihood that a response worker at the Fukushima Daiichi nuclear plant would ever benefit from autologous HPC transplantation? No one can say with certainty, but several arguments suggest that it is extraordinarily low. First, worker safety is the primary focus during clean-up of a stabilized radiation accident. According to extensive registries in the US and Europe, there have been no significant radiation exposures during the clean-up phase of any industrial radiation accident, including Chernobyl. ${ }^{3-4}$

Second, accidental radiation exposures are commonly very heterogeneous across the body (Figure 1)..$^{5}$ Dose heterogeneity can result from partial shielding by walls, furniture or equipment. Even a small fraction of shielded bone marrow can reconstitute hematopoiesis and thereby obviate the need for autologous HPC transplantation. Hypothetical scenarios have been proposed where a response worker must be exposed to life-threatening doses of radiation (e.g. to ensure a water or power supply). Such scenarios would be completely unprecedented, but even if necessary, a small amount of lead draping around the hips could provide protection to a region of bone marrow.

Third, previous radiation exposures that were severe enough to warrant HPC transplantation have been invariably lethal. The SEARCH database contains records from over 800 radiation casualties. ${ }^{4}$ Among the 45 who received myeloablative doses of radiation, all
45 died within 60 days. Multi-organ failure was extremely common and most had severe gastrointestinal, cutaneous and neurovascular radiation injuries. ${ }^{6}$ It is doubtful that autologous HPC transplantation would have offered benefit in these cases. Among 65 casualties who received doses that induced significant myelosuppression but not myeloblation, all 65 recovered normal blood counts within $60-80$ days $^{6}$. Even cases with persistent aplasia beyond 21 days after exposure can rapidly respond to myeloid cytokines (Figure 1). ${ }^{7}$ Fourth, myeloid cytokines, such as G-CSF and GM-CSF can hasten granulocyte recovery in radiation accident victims. Both G-CSF and GM-CSF improve overall survival in nonhuman primate models of radiation exposure. ${ }^{8-9}$ With the use of cytokines like G-CSF, bone marrow recovery may not be the survival-limiting factor after high-dose exposure. For example, a radiation casualty in Belarus was exposed to at least $10 \mathrm{~Gy}$ of whole-body radiation. After treatment with GM-CSF and IL-3, he recovered myeloid function but succumbed to radiation pneumonitis. ${ }^{10}$

Fifth, the cumulative experience with HPC transplantation after radiation accidents has been dismal. Approximately 40 radiation casualties have received allogeneic HPC transplants and all have either died from other injuries or recovered autologous hematopoeisis. ${ }^{11-12}$ Although autologous HPC transplantation has significantly less toxicity than allogeneic, there is currently no evidence that exogenous HPCs of any type can salvage human accident victims with otherwise lethal radiation injuries.

Preemptive HPC collection and banking for clean-up workers would consume substantial effort and resources. On the other hand, clean-up workers are providing an invaluable and possibly perilous service. One could argue that society is obligated to offer any approach of potential benefit, even if the potential is exceedingly small. The counterargument is that offering an unproven approach, regardless of resource requirements, could do more harm than good. Although generally well-tolerated, the administration of

Figure 1. An irradiated casualty from an accident in Fleurus, Belgium in 2006. Physical dosimetry on the left demonstrates the extensive heterogeneity of radiation exposure across the body. On the right, the time course after exposure demonstrates prolonged leucopenia that rapidly responded to pegylated granulocyte-colony stimulating factor (PEG-G-CSF) and other cytokines. The figure is modified from Gourmelon et al. $^{7}$

Biological Dosimetry Mean Dose: 4.2-4.8 Gy

Physical Dosimetry

Mean Dose : 4.2 - 4.8 Gy

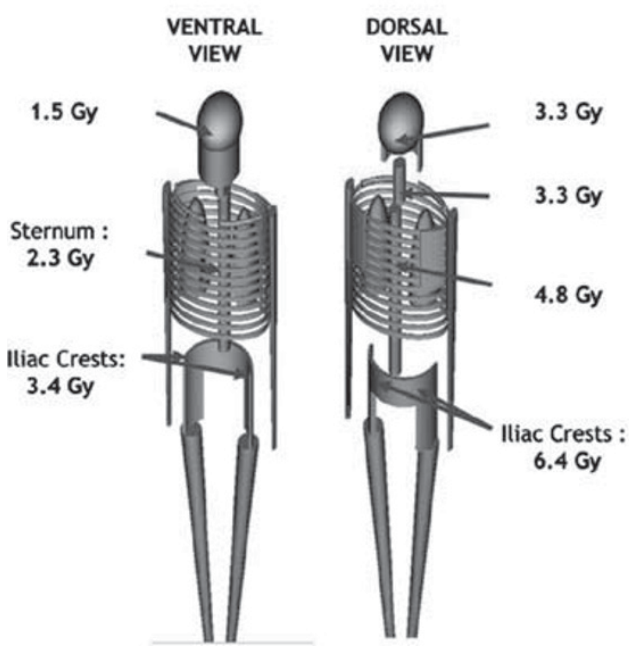


G-CSF to peripherally harvest HPCs is associated with significant rates of bone pain and constitutional symptoms. Severe complications like splenic rupture are rare but do occur. Theoretically, exposing workers treated with G-CSF to low dose irradiation may be particularly unsafe for some period, as G-CSF could induce cell division within damaged progenitors. Thus, workers who received G-CSF to harvest HPCs would require a wash-out window prior to radiation exposure. The lesson we have learned from exogenous erythropoietin, ${ }^{13}$ i.e. that treatments may seem innocuous but rarely are, certainly applies in this context.

The clean-up at Fukushima could involve hundreds or even thousands of workers over the course of decades. No objective basis exists for delineating the appropriate subset of workers to offer preemptive HPC banking. Should anyone involved in any aspect of the clean-up have the opportunity to bank HPCs or only those with certain responsibilities? Considering that any scenario leading to a high-dose exposure during the clean-up phase is predicated on the occurrence of an unanticipated complication, it seems counterintuitive that one could accurately predict who is most likely to be involved in these complications.

At the COSTEM meeting on September 9, 2011, I argued that the emphasis for radiation response should be on proven approaches for preventing and mitigating radiation injury. These include advanced exposure monitoring, supportive care, and radiation countermeasures like cytokines.

Acknowledgments: I thank Mr. Cullen Case and Drs. Dennis Confer Nelson Chao and Daniel Weisdorf for review of the manuscript and Dr. Weisdorf for the idea of partial-body lead shielding.

Disclosures: D.M.W. has received fees for consulting from Genzyme, Inc.

\section{References}

1. Tanimoto T, Uchida N, Kodama Y, Teshima T, Taniguchi S. Safety of workers at the Fukushima Daiichi nuclear power plant. Lancet. Apr 152011.

2. DiCarlo AL, Maher C, Hick JL et al. Radiation injury after a nuclear detonation: medical consequences and the need for scarce resources allocation. Disaster Med Public Health Prep. Mar 2011; 5 Suppl 1:S32-44.

3. REAC/TS Radiation Accident Registry: Update of Accidents in the United States. http://www.irpa.net/irpa10/cdrom/00325.pdf.

4. Friesecke I, Beyrer K, Wedel R, Reimers K, Fliedner TM. SEARCH: a system for evaluation and archiving of radiation accidents based on case histories. Radiat Environ Biophys. Sep 2000; 39(3):213-217.

5. Friesecke I, Beyrer K, Fliedner TM. How to cope with radiation accidents: the medical management. Br J Radiol. Feb 2001; 74(878):121-122.

6. Fliedner TM, H DD, Meineke V. Multi-organ involvement as a pathogenetic principle of the radiation syndromes: a study involving 110 case histories documented in SEARCH and classified as the bases of haematopoietic indicators of effect. BJR Supp/ 2005; 27:1-8

7. Gourmelon P, Benderitter M, Bertho JM, Huet C, Gorin NC, De Revel $\mathrm{P}$. European consensus on the medical management of acute radiation syndrome and analysis of the radiation accidents in Belgium and Senegal. Health Phys. Jun 2010; 98(6):825-832.

8. Farese AM, Williams DE, Seiler FR, MacVittie TJ. Combination protocols of cytokine therapy with interleukin-3 and granulocyte-macrophage colony-stimulating factor in a primate model of radiation-induced marrow aplasia. Blood. Nov 15 1993; 82(10):3012-3018.

9. MacVittie TJ, Farese AM, Jackson W, 3rd. Defining the full therapeutic potential of recombinant growth factors in the post radiation-accident environment: the effect of supportive care plus administration of G-CSF. Health Phys 2005; 89(5):546-555

10. Baranov AE, Selidovkin GD, Butturini A, Gale RP. Hematopoietic recovery after 10-Gy acute total body radiation. Blood Jan 15, 1994; 83(2): 596-599.

11. Dainiak N, Ricks RC. The evolving role of haematopoietic cell transplantation in radiation injury: potentials and limitations. BJR Supp/ 2005; 27 169-174.
12. Klymenko SV, Belyi DA, Ross JR et al. Hematopoietic cell infusion for the treatment of nuclear disaster victims: New data from the Chernobyl accident. Int J Radiat Biol Mar 16, 2011.

13. Buemi M, Campo S, Cernaro V, Donato V, Lacquaniti A. Erythropoietin and the truths of science. J Nephro/ Sep-Oct 2011; 24(5):564-568.

\section{The indication for unrelated allo-SCT in CR1 AML: "No"}

I Yakoub-Agha

Service des Maladies du Sang, CHU, Lille, France

The optimal treatment for acute myeloid leukemia (AML) remains to be defined. Although allogeneic hematopoietic stem cell transplantation (allo-SCT) represents a curative option for patients with $A M L$, allo-SCT from an unrelated donor should not be offered systematically to patients with AML in first complete remission (CR1) for two main reasons. Firstly, some subcategories of AML patients can potentially be cured with chemotherapy alone. Secondly, allo-SCT may be associated with a high risk of morbidity and mortality. Thus, several factors have to be taken into consideration before referring a patient for allo-SCT with an unrelated donor, ${ }^{1-3}$ including baseline cytogenetics, ${ }^{4,5}$ gene mutations in cytogenetically normal AML cells (including the genes for NPM1, FLT3-ITD, CEPBA, MLL and NRAS) and the refractoriness to the first cycle of high-dose chemotherapy. The donor type, the extent of HLA matching and the circumstances of the transplantation also influence the outcome after allo-SCT.-8

\section{Leukemia risk-status}

Good or intermediate-1 risk AML:This category includes core binding factor leukemia and cytogenetically normal $\mathrm{AML}$ that are $N P M 1^{+}$or CEBPA ${ }^{+}$but negative for FLT3-ITD.9,10 According to the main metaanalyses of prospective trials and a number of other studies, alloSCT is not indicated in CR1 AML - even when an HLA-matched sibling donor is available. , $^{611}, 12$

Poor-risk AML: This category comprises AMLs with 5q-, 7q-, 11q23 or MLL+, including MLL-PTD (except 9;11), t(6;9), 3q26 abs or EVI$1^{+}$and complex karyotype. ${ }^{9,10}$ In addition, patients requiring more than one course of induction chemotherapy to achieve CR should also be considered as falling into this category. ${ }^{2}$ Several studies have confirmed the indication of allo-SCT in CR1 in these patients, regardless of the type of donor and HLA matching. ${ }^{6,12-15}$

Intermediate- 2 risk AML: Almost $50 \%$ of patients with $\mathrm{AML}$ belong to this risk category. ${ }^{10}$ Although allo-SCT from an HLA-sibling is a good indication in patients with int-2 AML, 6,12 allo-SCT from an unrelated donor is still controversial - particularly in the case of an HLA-mismatch, since the outcome depends on the degree of donor-recipient HLA matching. Lee et al. ${ }^{7}$ reported that in patients with low- or intermediate-risk malignancies, survival was correlated with the number of HLA-mismatched antigens. On the other hand, the studies supporting the indication of allo-SCT from an unrelated donor all suffered from limitations that prevent robust conclusions from being drawn. Although the study we published several years ago concluded that transplantations from unrelated donors and from HLA-identical sibling donors had similar outcomes, no conclusions can be drawn with certainty concerning patients having received a peripheral blood graft or those with a non-myeloablative conditioning regimen, since (i) all the patients in our study had received a marrow graft and (ii) myeloablative conditioning consisted of total-body irradiation and cyclophosphamide. ${ }^{8}$ In addition, there were few patients with CR1 AML. A retrospective study by Walter et al. concluded that allo-SCT from unrelated donors could result in long-term survival for CR1 AML patients. However, interpretation of the study data is complicated by fact that $39 \%$ of the included patients had poor-risk AML and $30 \%$ required at least two induction courses to achieve CR. ${ }^{16}$

\section{The recipient's age}

Another factor that has to be taken into account is the recipient's age, since allo-SCT does not appear to be beneficial for elderly 
patients. ${ }^{6,13}$ It is difficult to unhesitatingly recommend reducedintensity conditioning in those patients, in view of the lack of randomized, prospective studies comparing this regimen to myeloablative conditioning. Furthermore, interpretation of the available data from retrospective studies is difficult, since the latter featured a mixture of older patients and poor-risk younger patients.

In conclusion, there is no firm indication (outside clinical trials) for allo-SCT from an unrelated donor in CR1 AML - except for patients with poor-risk AML.

\section{References}

1. Bullinger L, Dohner K, Kranz R et al. An FLT3 gene-expression signature predicts clinical outcome in normal karyotype AML. Blood 2008;111: 4490-4495.

2. Ravandi F, Cortes J, Faderl S et al. Characteristics and outcome of patients with acute myeloid leukemia refractory to 1 cycle of high-dose cytarabine-based induction chemotherapy. Blood 116:5818-5823; quiz 6153.

3. Schlenk RF, Dohner K, Krauter J et al. Mutations and treatment outcome in cytogenetically normal acute myeloid leukemia. N Engl J Med 2008, 358:1909-1918.

4. Dohner H, Estey EH, Amadori S et al. Diagnosis and management of acute myeloid leukemia in adults: recommendations from an international expert panel, on behalf of the European LeukemiaNet. Blood;115:453-474.

5. Taskesen E, Bullinger $L$, Corbacioglu A et al. Prognostic impact, concurrent genetic mutations, and gene expression features of AML with CEBPA mutations in a cohort of 1182 cytogenetically normal AML patients: further evidence for CEBPA double mutant AML as a distinctive disease entity. Blood 117:2469-2475.

6. Cornelissen JJ, van Putten WL, Verdonck LF et al. Results of a HOVON/ SAKK donor versus no-donor analysis of myeloablative HLA-identical sibling stem cell transplantation in first remission acute myeloid leukemia in young and middle-aged adults: benefits for whom? Blood 2007; 109:3658-3666.

7. Lee SJ, Klein J, Haagenson M et al. High-resolution donor-recipient HLA matching contributes to the success of unrelated donor marrow transplantation. Blood 2007; 110:4576-4583.
8. Yakoub-Agha I, de La Salmoniere P, Ribaud P et al. Allogeneic bone marrow transplantation for therapy-related myelodysplastic syndrome and acute myeloid leukemia: a long-term study of 70 patients-report of the French society of bone marrow transplantation. J Clin Oncol 2000; 18:963-971.

9. Slovak ML, Kopecky KJ, Cassileth PA et al. Karyotypic analysis predicts outcome of preremission and postremission therapy in adult acute myeloid leukemia: a Southwest Oncology Group/Eastern Cooperative Oncology Group Study. Blood 2000; 96:4075-4083.

10. Thomas X, Elhamri M, Raffoux E et al. Comparison of high-dose cytarabine and timed-sequential chemotherapy as consolidation for younger adults with AML in first remission: the ALFA-9802 study. Blood 118:1754-1762.

11. Suciu S, Mandelli F, de Witte T et al. Allogeneic compared with autologous stem cell transplantation in the treatment of patients younger than 46 years with acute myeloid leukemia (AML) in first complete remission (CR1): an intention-to-treat analysis of the EORTC/GIMEMAAML-10 trial. Blood 2003; 102:1232-1240.

12. Koreth J, Schlenk R, Kopecky KJ et al. Allogeneic stem cell transplantation for acute myeloid leukemia in first complete remission: systematic review and meta-analysis of prospective clinical trials. JAMA 2009; 301:2349-2361.

13. Gupta V, Tallman MS, He W et al. Comparable survival after HLA-wellmatched unrelated or matched sibling donor transplantation for acute myeloid leukemia in first remission with unfavorable cytogenetics at diagnosis. Blood 116:1839-1848.

14. Schlenk RF, Dohner K, Mack S et al. Prospective evaluation of allogeneic hematopoietic stem-cell transplantation from matched related and matched unrelated donors in younger adults with high-risk acute myeloid leukemia: German-Austrian trial AMLHD98A. J Clin Oncol 28: 4642-4648.

15. Burnett AK, Hills RK, Milligan DW et al. Attempts to optimize induction and consolidation treatment in acute myeloid leukemia: results of the MRC AML12 trial. J Clin Oncol 28: 586-595.

16. Walter RB, Pagel JM, Gooley TA et al. Comparison of matched unrelated and matched related donor myeloablative hematopoietic cell transplantation for adults with acute myeloid leukemia in first remission. Leukemia 24:1276-1282. 